\title{
Regulation of nitrogen fixation in Klebsiella pneumoniae: The role of Fnr in oxygen signal-transduction
}

\author{
Dissertation \\ Zur Erlangung des Doktorgrades \\ der Mathematisch-Naturwissenschaftlichen Fakultäten \\ der Georg-August Universität zu Göttingen
}

vorgelegt von

\section{Roman Grabbe}

aus Helmarshausen

Göttingen 2002 
Die vorliegende Arbeit wurde am Lehrstuhl für Allgemeine Mikrobiologie im Institut für Mikrobiologie und Genetik der Georg-August Universität Göttingen angefertigt.

Finanziert wurde diese Arbeit aus Mitteln der Deutschen Forschungsgemeinschaft im Schwerpunkt „Regulatorische Netzwerke in Bakterien“ und dem Fonds der chemischen Industrie.

Teile dieser Arbeit wurden veröffentlicht in:

Grabbe, R., Kuhn, A., and Schmitz, R.A. 2001a. Cloning, sequencing and characterization of Fnr from Klebsiella pneumoniae. Antonie Van Leeuwenhoek 79: 319-326.

Grabbe, R., Klopprogge, K., and Schmitz, R.A. 2001b. Fnr is Required for NifL-dependent oxygen control of nif gene expression in Klebsiella pneumoniae. J. Bacteriol. 183: 1385-1393.

Klopprogge, K., Grabbe, R., Hoppert, M., and Schmitz, R.A. 2002. Membrane association of Klebsiella pneumoniae NifL is affected by molecular oxygen and combined nitrogen. Arch. Microbiol. 177(3): 223-34.

Grabbe, R., and Schmitz, R.A. 2002. Oxygen Control of nif Gene Expression in Klebsiella pneumoniae is dependent on NifL reduction at the cytoplasmic membrane by electrons derived from the reduced quinone pool. (submitted)

Außerdem gingen folgende Veröffentlichungen aus der Arbeit hervor:

Ehlers, C, Grabbe R, Veit K, Schmitz RA 2002.Characterization of GlnK1 from Methanosarcina mazei strain Go1: complementation of an Escherichia coli glnK mutant strain by GlnK1. J. Bacteriol. 184(4): 1028-40.

D7

Referent: Prof. Dr. G. Gottschalk

Korreferent: PD. Dr. R.A. Schmitz-Streit

Tag der mündlichen Prüfung: 20.06.02 


\section{Acknowledgements}

Especially I would like to thank PD Dr. Ruth Schmitz for supervising and supporting this thesis. It has not always been easy to work with you because of your high demands on the people in the lab. But finally, I do believe that I will never learn more about science than I did under your inspiring guidance.

I thank Prof. Dr. G. Gottschalk for generous support and helpful discussions.

Special thanks to the former and current members of lab. 214: Anita, Anne, Christian, Claudia, Daniela, Edna, Jessica, Julia, Jutta, Kai, Katharina, Katja, Korinna, Melanie. It has been a pleasure working in such a friendly atmosphere. I will always remember that when looking back.

I like to thank all the people at the Institute for Microbiology and Genetics, Göttingen, especially the members of LII, G2L, the workshop and Mr. Hellwig.

Additionally, I want to thank my family and friends for giving me help and support.

Finally, I like to thank Tanja, just for being there. 


\section{Table of contents}

$\begin{array}{ll}\text { Summary } & 1\end{array}$

Chapter 1:

$\begin{array}{ll}\text { Introduction } & 3\end{array}$

NifL modulates NifA transcriptional activity by direct protein protein interaction $\quad 4$

Nitrogen signal transduction $\quad 5$

$\begin{array}{ll}\text { NifL response to molecular oxygen } & 6\end{array}$

Chapter 2:

Cloning, sequencing and characterization of Fnr from

$\begin{array}{lr}\text { Klebsiella pneumoniae } & 8\end{array}$

$\begin{array}{lr}\text { Abstract } & 8\end{array}$

$\begin{array}{ll}\text { Introduction } & 8\end{array}$

Materials \& Methods $\quad 9$

$\begin{array}{ll}\text { Results \& Discussion } & 15\end{array}$

$\begin{array}{ll}\text { Summary } & 19\end{array}$

Chapter 3:

Fnr is required for NifL dependent oxygen control of nif gene

expression in Klebsiella pneumoniae 21

$\begin{array}{ll}\text { Abstract } & 21\end{array}$

$\begin{array}{ll}\text { Introduction } & 22\end{array}$

Materials \& Methods 23

$\begin{array}{ll}\text { Results } & 30\end{array}$

$\begin{array}{ll}\text { Discussion } & 41\end{array}$ 
Chapter 4:

Membrane association of Klebsiella pneumoniae NifL is effected by molecular oxygen and combined nitrogen

$\begin{array}{ll}\text { Abstract } & 45\end{array}$

$\begin{array}{ll}\text { Introduction } & 45\end{array}$

Materials \& Methods $\quad 47$

Results

$\begin{array}{ll}\text { Discussion } & 63\end{array}$

Chapter 5:

Oxygen control of nif gene expression in Klebsiella pneumoniae is dependent on NifL reduction at the cytoplasmic membrane by $\begin{array}{ll}\text { electrons derived from tne reduced quinone pool } & 67\end{array}$

$\begin{array}{ll}\text { Abstract } & 67\end{array}$

$\begin{array}{ll}\text { Introduction } & 68\end{array}$

Materials \& Methods $\quad 70$

Results

$\begin{array}{lr}\text { Discussion } & 88\end{array}$

$\begin{array}{ll}\text { Conclusions } & 93\end{array}$

$\begin{array}{ll}\text { Current working model } & 96\end{array}$

$\begin{array}{ll}\text { Further studies } & 98\end{array}$

$\begin{array}{lr}\text { References } & 99\end{array}$

$\begin{array}{ll}\text { Curriculum vitae } & 114\end{array}$ 


\section{Summary}

- In the free-living diazotroph Klebsiella pneumoniae, a member of the $\gamma$-subgroup of Proteobacteria, nitrogen fixation (nif) genes are under the control of the nifLA operon, the products of which regulate transcription of the nif operons. NifA activates nif gene transcription by alternative RNA polymerase, $\sigma^{54}$-holoenzyme; the negative regulator NifL modulates activity of NifA in response to molecular oxygen and combined nitrogen. Transcriptionally coupled synthesis, immunological studies and complex analysis of both regulators indicate that NifL-mediated inhibition of NifA depends on direct proteinprotein interaction.

- The negative regulator NifL is a flavoprotein, which modulates NifA activity depending on the redox state of its N-terminally bound FAD-cofactor. Thus, oxygen might be sensed directly by the redox-sensitive cofactor of NifL or by a global oxygen sensor, for example Fnr (fumarate nitrate reductase regulator), which transduces the oxygen signal towards the NifL-bound cofactor.

- The fnr gene of K. pneumoniae was cloned, sequenced and biochemically analyzed. The analysis of the deduced amino acid sequence revealed $98 \%$ similarity to the Escherichia coli Fnr protein. The conserved cystein residues, which establish the oxygen-sensing [4Fe-4S]-cluster, are located in the N-terminal domain of the K. pneumoniae Fnr as it is kown for the E. coli protein. Biochemical analysis of the glutathionS-transferase (GST) fusion protein Fnr-GST expressed and purified under aerobic or anaerobic conditions, revealed decreased amounts of iron and acid-labile sulphur in the aerobic protein compared to the anaerobic protein. This indicates that $K$. pneumoniae Fnr sesnes oxygen based on an oxygen-sensitive iron-sulphur cluster.

- Studying the oxygen dependent regulation of nif induction in fnr mutant backgrounds we obtained strong evidence that in K. pneumoniae Fnr is the primary oxygen sensor for the nif regulatory system. In the absence of Fnr, NifL did not receive the signal of anaerobiosis under nitrogen and oxygen limited conditions resulting in a decreased NifA activity. Thus, Fnr appears to sense the oxygen status of the cell and presumably transduces the signal of anaerobiosis towards Nifl by activating gene(s), the product(s) of which function to reduce the FAD cofactor of NifL resulting in a non-inhibitory conformation. Attractive candidates for the physiological electron donor for NifL 
reduction are components of the anaerobic electron transport chain, which are Fnrdependent transcribed.

- Localization experiments of NifL in K. pneumoniae under different growth conditions revealed that NifL is highly membrane associated under derepressing growth conditions. However, when cells were shifted to ammonium sufficiency or presence of oxygen NifL is located in the cytoplasm. Further studies using K. pneumoniae mutant strains showed that under derepressing conditions but in the absence of either Fnr or the nitrogen sensor GlnK NifL was located in the cytoplasm and inhibited NifA activity. Presumably in the absence of Fnr or GlnK NifL does not receive the signal of anaerobiosis or nitrogen limitation. In contrast to NifL, NifA remains in the cytoplasm under all conditions tested. Thus, sequestration of NifL to the membrane under nitrogen and oxygen-limitation is involved in the mechanism of NifA regulation.

- Biochemical analysis of purified NifL showed that NifL-bound FAD-cofactor was reduced by $\mathrm{NADH} / \mathrm{H}^{+}$only in the presence of a redox mediator or inside-out vesicles derived from anaerobically grown $K$. pneumoniae cells. This indicates that in vivo NifL is reduced at the cytoplasmic membrane.

- In order to identify the physiological electron donor for NifL reduction, the effect of different oxidoreductase systems on nif regulation was studied in the respective mutant backgrounds. Using K. pneumoniae mutant strains we observed strong evidence, that in the absence of a functional NADH:ubiquinone oxidoreductase or formate dehydrogenaseN NifL inhibition of NifA was not relieved. The same effect was observed in a heterologous $E$. coli system lacking the alternative NADH dehydrogenase $(n d h)$. Further studies of nif induction of anaerobically grown cultures on glycerol showed significantly reduced NifA activity when nitrate was added as additional electron acceptor. Taking together these findings indicate that more than one oxidoreductase system appears to be responsible for NifL reduction and that NifL receives electrons from the reduced quinone pool.

- We further demonstrated that reduced dimethylnaphthoquinone $\left(\mathrm{DMN}_{\mathrm{red}}\right)$, a soluble quinone derivative is able to reduce the FAD cofactor of NifL in the absence of a redox mediator. This finding supports our model that the cofactor FAD of the membraneassociated NifL receives electrons from the reduced quinone pools, generated by different oxidoreductase systems. 


\section{Chapter 1:}

\section{Introduction}

Biological nitrogen fixation, the enzymatic reduction of molecular nitrogen $\left(\mathrm{N}_{2}\right)$ to ammonia, is strictly limited to prokaryotes. However, within the prokaryotes nitrogen fixation is found in a large number of species belonging to the bacterial domain and in several methanogenic Archaea (Dean and Jacobson, 1992; Young, 1992; Lobo and Zinder, 1992; Fischer, 1994; Galagan et al., 2002; Deppenmeier et al., 2002). The reduction of molecular nitrogen is catalyzed by the nitrogenase enzyme complex with high energy demands. Two ATP molecules are consumed for each electron transferred to the catalytic site (Burgess and Lowe, 1996; Howard and Rees, 1996; Rees and Howard, 1999, Halbleib and Ludden, 2000). Because of the high energy requirement for $\mathrm{N}_{2}$ fixation, up to $40 \%$ of the ATP is utilized by the nitrogenase in nitrogen fixing cells, resulting in a drop of the energy charge from 0.9 to 0.5 (Daesch and Mortenson, 1972; Upchurch et al., 1980). In the presence of molecular oxygen the nitrogenase enzyme complex is irreversibly inactivated. Thus, to avoid unnecessary consumption of energy nitrogen fixing microorganisms tightly control synthesis and activity of nitrogenase in response to nitrogen and oxygen availability. In all diazotrophic proteobacteria examined, the transcriptional activator NifA is required for transcription of the nitrogen fixation (nif) genes. NifA expression and activity is regulated in response to the environmental signals, molecular oxygen and combined nitrogen. However, the mechanisms of NifA regulation vary in different organisms (Fischer, 1996; Dixon, 1998; Halbleib and Ludden, 2000; Schmitz et al., 2002). In free-living and symbiotic diazotrophs belonging to the $\alpha$-and $\beta$-subgroup of the proteobacteria (genera Rhizobium, Bradyrhizobium, Azospirillum and Herbaspirillum) NifA activity is directly sensitive to molecular oxygen and in some cases affected in the presence of combined nitrogen (Fischer, 1994; Fischer, 1996; Steenhoudt and Vanderleyden, 2000). In contrast, in Klebsiella pneumoniae and Azotobacter vinelandii, two free-living diazotrophs, which belong to the $\gamma$-proteobacteria, NifA activity is not oxygen sensitive. NifA activity is regulated in response to molecular oxygen and fixed nitrogen by a second regulator NifL, the gene of which forms an operon with nifA (Filser, 1983; Dixon, 1998). In K. pneumoniae the expression of the nifLA operon itself is regulated by the nitrogen status, via the $\mathrm{NtrB} / \mathrm{NtrC}$ two component regulatory system, whereas in $A$. vinelandii nifLA is constitutively expressed (Drummond and Wootton, 1987; Blanco et al., 1993). Interestingly, it was recently found that nitrogen fixation of the endophytic diazotroph Azoarcus spec. belonging to the $\beta$-proteobacteria - is also regulated by the coordinated activities of nifL and 
nifA gene products in response to environmental signals (Egener and Reinhold-Hurek, unpublished).

NifL modulates NifA transcriptional activity by direct protein-protein interaction. The transcriptional activator NifA is composed of three domains: an amino (N)-terminal domain apparently involved in the regulation, a central catalytic domain, and a carboxy (C)-terminal DNA-binding domain (Drummond et al., 1990; Morett and Segovia, 1993). Transcription of nif genes by the alternative RNA polymerase ( $\sigma^{54}$-RNA polymerase) is generally activated by NifA, which binds to an upstream activation sequence (UAS) (Morrett and Buck, 1988) and contacts promoter-bound $\sigma^{54-R N A ~ p o l y m e r a s e ~ b y ~ m e a n s ~ o f ~ a ~ D N A ~ l o o p ~(B u c k ~ e t ~ a l ., ~ 1987) . ~}$ Subsequently NifA catalyzes the isomerization of closed complexes between $\sigma^{54}$-holoenzyme and the nif promoter to transcriptionally productive open complexes (Morett and Buck, 1989; Hoover et al., 1990). This open complex formation requires hydrolysis of ATP or GTP catalyzed by NifA (Lee et al., 1993; Austin et al., 1994). In the presence of molecular oxygen or combined nitrogen, NifL inhibits NifA activity in vivo (Merrick et al., 1982; Hill et al., 1981; Dixon, 1998; Schmitz et al. 2002). The inhibitory protein NifL is composed of two domains separated by a hydrophilic interdomain linker (Q-linker) (Söderbäck et al., 1998; Drummond and Wootton, 1987). The C-terminal domain of NifL shows homology to a histidine protein kinase (Blanco et al., 1993). However, neither autophosphorylation nor possible phosphor transfer between the two regulatory proteins NifA and NifL has been detected in K. pneumoniae or A. vinelandii (Lee et al., 1993; Austin et al., 1994; Schmitz et $a l ., 1996)$. The translationally coupled synthesis of nifL and nif $A$ and immunological studies imply that the inhibition of NifA activity by NifL apparently occurs via a direct proteinprotein interaction (Govantes et al., 1998; Henderson et al., 1989). Recently, complex formation between $A$. vinelandii NifL and NifA has been demonstrated by in vitro cochromatography in the presence of adenosine nucleotides and using the yeast two hybrid system (Money et al., 2001 and 1999; Lei et al., 1999). Thus, signal transduction apparently occurs via protein-protein interaction. Interestingly, for $A$. vinelandii it was shown that NifL influences both NifA transcriptional activity and DNA-binding capacity in vitro (Barrett et al., 2001). The C-terminal domain of K. pneumoniae NifL is sufficient to inhibit transcriptional activation by NifA in vitro and in vivo (Narberhaus et al., 1995). This indicates that the inhibitory function of NifL protein appears to be located in its C-terminal domain, which presumably interacts with NifA by protein-protein interaction. 
Nitrogen signal transduction. In K. pneumoniae, a shift from nitrogen limitation to nitrogen sufficiency results in repression of nif gene induction upon inhibition of NifA transcriptional activity by NifL (Arnott et al. 1989; Blanco et al., 1993). This indicates that NifL either senses the nitrogen availability directly or the nitrogen status is sensed in a NifL independent manner and the signal is subsequently transduced to NifL or the NifL/NifA complex. Interestingly, like in Escherichia coli a second PII-like protein, encoded by $g \ln K$, was recently discovered in K. pneumoniae. $g \ln K$ is organized with $a m t B$ (encoding for an ammonium transporter) in an operon, which is under transcriptional control of NtrC. Upon the high similarity to the PII-protein, the GlnK-protein is an attractive candidate for sensing changes in the glutamine pool size - reflecting the internal nitrogen status - and mediating the signal of the nitrogen status to the nif regulatory system (Atkinson and Ninfa, 1998; Xu et al., 1998; van Heeswijk et al., 1996). Studying nif regulation in $g \ln K$ mutant strains strong evidence was obtained, that GlnK is indeed required to release NifL inhibition under nitrogen-limiting growth conditions in K. pneumoniae (He et al., 1998; Jack et al., 1999; Arcondeguy et al., 1999). This indicates that changes of the internal nitrogen status are not sensed by NifL directly, but are apparently mediated by GlnK to the NifA/NifL regulatory system. Whereas NifL is a negative regulator, GlnK acts positively to antagonize inhibitory effects of NifL under nitrogen-limiting conditions. The uridylylation status of GlnK is probably not required for relief of NifL inhibition (He et al. 1998; Arcondeguy et al., 1999). Interestingly, the Tloops of GlnK and PII from K. pneumoniae, which are supposed to interact with other components involved in the signal transduction, differ only in three amino acid residues 43 , 52 and 54. It has been shown that for regulation of the nif system residue 54 is the most important amino acid in the T-loop of GlnK, possibly directly involved in the interaction with NifL/NifA (Arcondeguy et al., 2000). Although GlnK function has been clearly demonstrated, the question arises, how GlnK is mediating the nitrogen signal towards the NifL/NifA regulatory system. The nitrogen signal is apparently mediated by direct protein-protein interaction but it has to be elucidated, whether GlnK is interacting directly with NifL or is affecting the NifL/NifA complex formation. For diazotrophs not belonging to the $\gamma$ proteobacteria and missing NifL (e.g. Herbaspirillum seropedicae and Azospirillum brasilense) experimental data indicate that the PII proteins participate in signaling the nitrogen status to the N-terminal domain of NifA (Steenhoudt and Vanderleyden, 2000; Souza et al., 1999; Monteiro et al., 1999, Arsene et al., 1999).

A. vinelandii contains only one PII-like protein, encoded in a $g \ln K / a m t B$-operon, which is expressed constitutively (Meletzus et al., 1998). Interestingly, A. vinelandii GlnK has a T- 
loop structure, which resembles more the 'GlnB-like' T-loop rather than the 'GlnK-like' T-loop (Arcondeguy et al., 2000). Recent studies concerning the role of $A$. vinelandii GlnK in nitrogen sensing and transducing the nitrogen status to the nif regulatory system showed that $\mathrm{GlnK}$ is not required for derepression in A. vinelandii. In contrary to $K$. pneumoniae, where GlnK apparently has a positive role in relieving NifL inhibition under nitrogen limiting conditions, in vitro experiments suggest that the inhibitory function of $A$. vinelandii NifL is activated under nitrogen excess through interaction with PII-like regulatory proteins (ReyesRamirez et al., 2000; Little et al., 2000 and 2002). Recently interactions between NifL and GlnK have been reported for $A$. vinelandii using the yeast two-hybrid system (Rudnick et al., 2002) and it was demonstrated in vitro that GlnK interacts with the C-terminal domain of NifL (Little et al., 2002). Dixon and coworker proposed that interaction with NifL only occurs when GlnK is not uridylylated and activates NifL inhibitory functions under nitrogen sufficiency (Little et al., 2002). This suggests that NifA inhibition by NifL is relieved when GlnK is uridylylated, but uridylylated GlnK is not required for this relief. However, very recently Merrick and coworkers showed that in E. coli and A. vinelandii non-uridylylated GlnK is highly membrane associated after a shift to nitrogen sufficiency upon binding to the ammonium transporter AmtB (Coutts et al., 2002) and thus, unmodified GlnK should not be available in the cytoplasm to activate NifL inhibitory functions.

NifL response to molecular oxygen. The N-terminal domain of NifL contains conserved Smotifs of PAS-like domains, which are known for a number of regulators sensing oxygen, redox or light (Zhulin et al., 1997; Taylor and Zhulin, 1999). This indicates that the Nterminal domain is involved in signal transduction. Biochemical analyses of purified proteins showed that NifL from $A$. vinelandii and from $K$. pneumoniae is a flavoprotein with an Nterminally bound FAD-cofactor (Hill et al., 1996; Schmitz, 1997; Söderbäck et al., 1998; Klopprogge and Schmitz, 1999). Analysis of the inhibitory function of NifL-holoenzyme and NifL-apoenzyme on NifA activity in in vitro transcription assays showed that the FADcofactor is not directly required for NifL inhibitory function (Schmitz, 1997). This indicates that FAD acts as a redox-sensitive cofactor, which might be involved in the oxygen signal transduction. The oxidized form of NifL inhibits NifA transcriptional activity in vitro, whereas A. vinelandii NifL reduced by sodium dithionite or by the flavoheme protein (Hmp) from $E$. coli with $\mathrm{NADH} / \mathrm{H}^{+}$as electron donor does not antagonize open complex formation by NifA in vitro (Macheroux et al., 1998). Thus, reduction of the flavin moiety of NifL results in a non-inhibitory form of NifL, however functional and physiological relevance for the 
reduction of NifL by Hmp, which is proposed to be a global oxygen sensor (Pool, 1994), has not been demonstrated to date. These findings support the model that NifL acts as a redoxsensitive regulatory protein that modulates NifA activity in response to the redox state of its FAD-cofactor and allows NifA activity only in the absence of oxygen. However, in both organisms the physiological electron donor for NifL is not known.

Reduction of the FAD-cofactor by the physiological electron donor apparently transduces the signal for anaerobiosis to NifL. As a consequence, components of the oxygen signal transduction are attractive candidates for the electron transfer towards NifL in vitro. Thus, the key question concerning the oxygen signal transduction is, whether NifL senses the oxygen status of the cell directly via a redox induced conformational change. Alternatively, oxygen might be detected by a more general oxygen-sensing system, which then regulates NifL by inducing the oxidation or reduction of the flavin cofactor. In this respect it is of interest that in $K$. pneumoniae, iron is specifically required for relief of NifL inhibition under oxygen and nitrogen limitation (Schmitz et al., 1996). The finding that $K$. pneumoniae NifL does not contain non-heme iron or an acid-labile sulphur cluster (Schmitz et al., 1996; Klopprogge and Schmitz 1999), indicates the presence an iron containing protein in the oxygen signal cascade towards NifL. In E. coli the transcriptional regulator Fnr (fumarate nitrate reductase regulator) plays an overarching role in sensing the switch from anaerobic to aerobic conditions. The mechanism of oxygen sensing in Fnr is mediated via an [4Fe-4S]-cluster (Green et al., 1996; Unden and Shirawski, 1997; Kiley and Beinert, 1998). Interestingly, in Rhizobium leguminosarum FnrN, a Fnr homologous protein, regulates nitrogen fixation in an oxygendependent manner (Gutierrez et al., 1997). Thus, it is attractive to speculate that a Fnr homologous protein is involved in oxygen-dependent regulation of nitrogen fixation in $K$. pneumoniae.

The intention of this thesis was to study the signal transduction of molecular oxygen towards NifL in K. pneumoniae. Investigations were performed to study (i) the role of Fnr in the oxygen-sensing mechanism for nitrogen fixation (chapter 2 and 3), (ii) the cellular localization of NifL followed by functional analyses of NifL localization for NifA regulation (chapter 4), and (iii) the effect of membrane-bound oxidoreductase systems concerning oxygen sensing on nif regulation (chapter 5). 


\title{
Chapter 2:
}

\section{Cloning, sequencing and characterization of Fnr}

\author{
from Klebsiella pneumoniae
}

\begin{abstract}
The transcription factor Fnr (fumarate nitrate reductase regulator) globally regulates gene expression in response to oxygen deprivation in Escherichia coli. We report here the cloning and sequencing of the fnr gene from the facultative anaerobic bacterium Klebsiella pneumoniae M5al, another member of the enteric bacteria. The deduced amino acid sequence of $K$. pneumoniae fnr showed very high similarity (98\% amino acid identity) to the Fnr protein from E. coli and contained the four essential cysteine residues which are presumed to build the oxygen-sensing $[4 \mathrm{Fe} 4 \mathrm{~S}]^{+2}$ center. Transfer of the K. pneumoniae gene to a fnr mutant of $E$. coli complemented the mutation and permitted synthesis of nitrate reductase and fumarate reductase during anaerobic growth. A gene fusion between $K$. pneumoniae fnr and glutathione S-transferase was constructed and expressed in E. coli under anaerobic conditions in order to make the protein available in preparative amounts. The overproduced protein was purified by glutathione-Sepharose 4B affinity chromatography in the absence of oxygen, and biochemically characterized.
\end{abstract}

\section{INTRODUCTION:}

Many of the oxygen-responsive gene regulators of bacteria are members of the fumarate nitrate reductase / cyclic AMP receptor protein family of transcriptional regulators (Spiro 1994, Gunsalus \& Park 1994, Unden et al. 1995). The fumarate nitrate reductase regulator from Escherichia coli $\left(\mathrm{Fnr}_{\mathrm{Ec}}\right)$ acts as a redox-responsive transcriptional regulator that activates genes whose products are involved in anaerobic respiration and represses other genes required for aerobic respiration (Spiro 1994, Gunsalus \& Park 1994, Unden et al. 1995, Bauer et al. 1999). It contains a cluster of three closely-spaced cysteine residues located near the $\mathrm{N}$-terminus $\left({ }^{20} \mathrm{CysX}_{2} \mathrm{CysX}_{5}{ }^{29} \mathrm{Cys}\right)$ plus an additional cysteine residue, Cys122. These cysteine residues are required for the oxygen-sensing function (Spiro \& Guest 1988). Recent data suggest that these residues bind an $[4 \mathrm{Fe} 4 \mathrm{~S}]^{+2}$-cluster and that this cluster apparently 
mediates the sensitivity of the transcriptional activator to oxygen (Green et al. 1996; Khoroshilova et al. 1997; Kiley \& Beinert 1998). In addition, the presence of the $[4 \mathrm{Fe} 4 \mathrm{~S}]^{+2}$ cluster in the anaerobically-purified form of Fnr is correlated with dimerization and specific DNA binding. Upon addition of oxygen, the $[4 \mathrm{Fe} 4 \mathrm{~S}]^{+2}$-cluster is disrupted, resulting in the conversion of Fnr into an inactive monomeric protein (Lazazzera et al. 1996; Melville \& Gunsalus 1996). Homologs of Fnr have been identified in several gram-negative and grampositive bacteria, some of which differ with respect to the cystein residues and the coordination of the iron-sulphur clusters (reviewed in Spiro 1994; Cruz Ramos et al. 1995; Saunders et al. 1999; Vollack et al. 1999). Recently discovered examples of Fnr homologues, which do not exhibit the structural elements or coordinate the iron-sulphur clusters differently are: (i) Fnr from Bacillus subtilis and B. licheniformis, for which a C-terminal cluster coordination is found (Cruz Ramos et al. 1995; Klinger et al. 1998); (ii) Fnr homologues from Lactobacillus casei and L. lactis, that lack two of the four essential cysteine residues and in the case of L. casei, Flp redox sensitive switch is operated based on a reversible interconversion of an intramolecular disulphide bridge (Gostick et al. 1998; Scott et al. 2000); and (iii) the Fnr homologues DnrD, DnrE and DnrS of Pseudomonas stutzeri, which completely lack the respective cysteine residues and iron-sulphur centres (Vollack et al. 1999).

Adaptation of the facultative anaerobic bacterium Klebsiella pneumoniae to anaerobic growth conditions is also accompanied by dramatic changes in metabolic gene expression. In addition, it is only when growing in the absence of molecular oxygen that $K$. pneumoniae is able to use molecular nitrogen as sole nitrogen source under nitrogen limitation (Dixon 1998). In order to make these adaptations, $K$. pneumoniae must sense changes in environmental oxygen availability. In contrast to $E$. coli, little is known about a regulatory oxygen-sensing system in Klebsiella. However, there are some evidences suggesting the presence of an Fnr-homologue in K. pneumoniae: Fnr is possibly involved in expression of the citrate-specific fermentation genes in K. pneumoniae (Bott et al. 1995) and in $K$. terrigena Fnr might act as a repressor of the butanediol (bud) operon (Mayer et al. 1995).

In this communication we report on the sequencing and characterization of the regulatory gene $f n r$ from $K$. pneumoniae. 


\section{Bacterial Strains and Plasmids.}

The bacterial strains and plasmids used in this work are listed in Table 1. Plasmid DNA was transformed into E. coli cells according to the method of Inoue et al. (1990) or by electroporation using a Gene pulser and Pulse controller (BioRad Laboratories). The fnr::Tn10 allele was transferred from the fnr::Tn10 derivative of M182 (Jayaraman et al. 1988) by P1-mediated transduction into NCM1529 and RM123 as described previously (Silhavy et al. 1984) with selection for tetracycline resistance; the resulting strain designated RAS1 and RAS6 respectively. Strains RAS3, RAS4 and RAS5 contain plasmids pRS120, pRS127 and pRS137, respectively, in RAS1; strain RAS21 contains pRS137 in RAS6. Plasmids pRS120 and pRS137 contain the E. coli fnr gene and K. pneumoniae fnr gene, respectively, inserted into the SalI and EcoRV site of pACYC184 and thereby expressed from the tet promoter.

\section{Media and growth conditions.}

For cloning, E. coli was routinely grown in LB medium at $37{ }^{\circ} \mathrm{C}$ (Ausubel et al. 1987). The medium was supplemented with ampicillin at $100 \mu \mathrm{g} / \mathrm{ml}$ or chloramphenicol at 15 $\mu \mathrm{g} / \mathrm{ml}$ to maintain recombinant plasmids; additionally, $5 \mu \mathrm{g} / \mathrm{ml}$ tetracycline was added to the growth medium when NCM1529(fnr::Tn10) or RM123(fnr::Tn10) were the host strains. For complementation experiments, strains were grown under anaerobic conditions with $\mathrm{N}_{2}$ as gas phase at $37{ }^{\circ} \mathrm{C}$ in minimal medium $\left(100 \mathrm{mM} \mathrm{KH}_{2} \mathrm{PO}_{4}, 50 \mathrm{mM} \mathrm{NaHPO}, 1 \mathrm{mM} \mathrm{MgSO} 4,0.1\right.$ $\mathrm{mM} \mathrm{CaCl}_{2}, 10 \mu \mathrm{M} \mathrm{Na}_{2} \mathrm{SeO}_{3}, 10 \mu \mathrm{M} \mathrm{Na}_{2} \mathrm{MoO}_{4}, 0.3 \mathrm{mM}$ sulfide and $0.002 \%$ resazurine (to monitor anaerobiosis) $\mathrm{pH}=6.5$ ), containing $0.8 \%$ glycerol as the $\mathrm{C}$-source and $1 \% \mathrm{KNO}_{3}$ as the only nitrogen source. Precultures were grown overnight in closed bottles, with $\mathrm{N}_{2}$ as gas phase, in medium lacking sulfide and resazurine, and additionally supplemented with $4 \mathrm{mM}$ ammonium acetate which was completely utilized after growth of the precultures to saturation. The main cultures $(25 \mathrm{ml})$ were inoculated from saturated precultures and were grown in closed bottles at $37^{\circ} \mathrm{C}$ without shaking.

\section{Construction of a gene library of $K$. pneumoniae chromosomal DNA.}

Chromosomal DNA from K. pneumoniae M5a1 was isolated according the method described by Ausubel et al. (1987). Fifty micrograms of DNA was partially digested with Sau3AI so that the majority of fragments were in the size range of between 20 and $30 \mathrm{kbp}$. The purified digested DNA was ligated to $1 \mu \mathrm{g}$ pWE15, which had been completely digested with $B a m H I$ and dephosphorylated. The ligation mixture was then packed and transduced 
into E. coli VCS257 using the Gigapack III Gold (Stratagene, La Jolla, US) packaging extract according the protocol of the manufacturer. Approximately 8000 colonies were collected.

\section{Generation of a $100 \mathrm{bp}$ hybridization probe for the fur gene from $K$. pneumoniae.}

A probe for the fnr gene was obtained by PCR using genomic DNA from $K$. pneumoniae as template. The oligonucleotides were derived from the E. coli fnr sequence: 5' primer (5'ATCAATTACGGATCCAGCAGACCTATGATCCCG3') and 3' primer (5'GTGTGAACG GGATCCAAAGCTGGC3'). Reactions were carried out in $100 \mu$ l volumes using Vent polymerase (New England Biolabs, UK) and primers at a concentration of $0.3 \mu \mathrm{M}$. The annealing temperature was at $65{ }^{\circ} \mathrm{C}$ and synthesis was carried out for $30 \mathrm{~s}$, for 25 cycles. The 100 bp PCR product was purified with Wizard ${ }^{\circledR}$ Plus PCR Purification system (Promega, Heidelberg, Germany) and labeled with the random Dig-labeling kit from Boehringer Mannheim according the protocol of the manufacturer. The specificity of the probe was tested by Southern hybridizations (Sambrook et al. 1989) with $K$. pneumoniae DNA digested completely digested by BamHI and EcoRI. Under the conditions employed, the hybridization with the labeled probe resulted in only one hybridization signal in each digest.

\section{TABLE 1: Bacterial strains and plasmids used in this study}

\begin{tabular}{|c|c|c|}
\hline$\underline{\text { Strains / plasmids }}$ & $\underline{\text { Relevant genotype and/or characteristic(s) }}$ & $\underline{\text { Reference or description }}$ \\
\hline \multicolumn{3}{|l|}{$\underline{\text { Strains }}$} \\
\hline $\mathrm{M} 182(f n r:: \operatorname{Tn} 10)$ & M182 but $f n r:: \operatorname{Tn} 10$ & Jayaraman et al. 1998 \\
\hline NCM1529 & 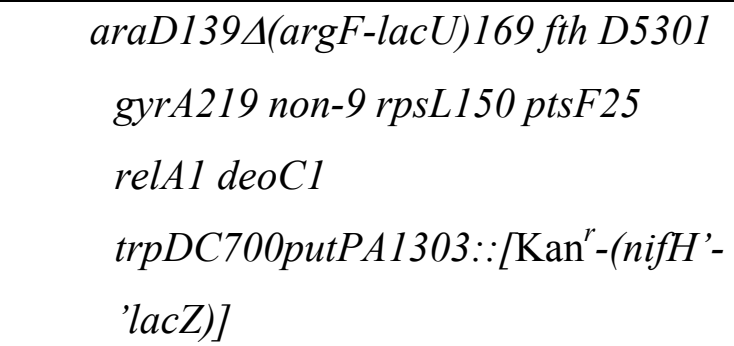 & He et al. 1997 \\
\hline RAS1 & NCM1529 but $f n r:: \operatorname{Tn} 10$ & \\
\hline
\end{tabular}




\begin{tabular}{|c|c|c|}
\hline & & $\begin{array}{l}\text { See Materials and } \\
\text { Methods }\end{array}$ \\
\hline RAS3 & RAS1/pRS120 & $\begin{array}{l}\text { See Materials and } \\
\text { Methods }\end{array}$ \\
\hline RAS4 & RAS1/pRS127 & $\begin{array}{l}\text { See Materials and } \\
\text { Methods }\end{array}$ \\
\hline RAS5 & RAS1/pRS137 & $\begin{array}{l}\text { See Materials and } \\
\text { Methods }\end{array}$ \\
\hline$\underline{\text { Plasmids }}$ & $\begin{array}{l}\text { Relevant genotype and / or } \\
\text { characteristic(s) }\end{array}$ & $\frac{\text { Reference or }}{\underline{\text { description }}}$ \\
\hline pWE15 & cosmid vector & $\begin{array}{l}\text { Stratagene, La Jolla, } \\
\text { US }\end{array}$ \\
\hline pBluescript SK+ & cloning vector & $\begin{array}{l}\text { Stratagene, La Jolla, } \\
\text { US }\end{array}$ \\
\hline pACYC184 & low copy vector & $\begin{array}{l}\text { New England Biolabs } \\
\text { (UK) }\end{array}$ \\
\hline pGEX-2T & $\begin{array}{l}\text { Expression vector, expression in } \\
\text { fusion with glutathione-S transferase }\end{array}$ & $\begin{array}{l}\text { Pharmacia, Freiburg } \\
\text { Germany }\end{array}$ \\
\hline pRS120 & $\begin{array}{l}\text { E. coli fnr controlled by the tet } \\
\text { promoter on pACYC184 }\end{array}$ & $\begin{array}{l}\text { See Materials and } \\
\text { Methods }\end{array}$ \\
\hline pRS127 & $\begin{array}{l}2.1 \mathrm{kbp} \text { fragment in pBluescript } \mathrm{SK}+ \\
\text { containing K. pneumoniae fnr }\end{array}$ & $\begin{array}{l}\text { See Materials and } \\
\text { Methods }\end{array}$ \\
\hline
\end{tabular}




\begin{tabular}{|c|c|c|}
\hline pRS131 & $\begin{array}{c}\text { K. pneumoniae fnr cloned into pGEX- } \\
\text { 2T under the control of the tac } \\
\text { promoter, coding for glutathione-S } \\
\text { transferase fused to Fnr }\end{array}$ & $\begin{array}{c}\text { See Materials and } \\
\text { Methods }\end{array}$ \\
\hline pRS137 & $\begin{array}{c}\text { K. pneumoniae fnr controlled by the } \\
\text { tet promoter on pACYC184 }\end{array}$ & $\begin{array}{c}\text { See Materials and } \\
\text { Methods }\end{array}$ \\
\hline
\end{tabular}

\section{Cloning and sequencing of $K$. pneumoniae fnr gene.}

Heterologous cosmids from the gene library of $K$. pneumoniae chromosomal DNA was completely digested by BamH1 and EcoRI. After blotting onto Nylon membrane Hybond-N (Amersham) and Southern hybridization (Sambrock et al. 1989) using the $100 \mathrm{bp}$ probe, the digested cosmids were screened for positives using the luminescent detection kit for nucleic acids from Boehringer Mannheim. Three positive cosmids were obtained and subcloned into $\mathrm{pSK}^{+}$Bluescript (Stratagene, La Jolla, US) resulting in plasmid pRS127, containing a $2.1 \mathrm{kbp}$ EcoRI/BamHI fragment which hybridized with the fnr probe. DNA sequences of both strands were determined independently and completely by commercial sequencing by MWG Biotech (Ebersberg, Germany). Sequence analysis was performed with the Genetics Computer Group (GCG) program package (Devereux et al. 1984).

\section{Enzyme activities.}

To determine synthesis of fumarate reductase by measuring fumarate reductase activity cells were grown in minimal medium (Schmitz et al. 1996) supplemented with $10 \mathrm{mM}$ ammonium, $1 \%$ glucose and $50 \mathrm{mM}$ fumarate. Cell extracts were prepared from anaerobically grown cells at an O.D. $600=0.6$. Cells were disrupted under anaerobic conditions in breakage buffer ( $50 \mathrm{mM}$ Tris/ $\mathrm{HCl}$ buffer $\mathrm{pH}=7.6$ containing $4 \mathrm{mM}$ dithiothreithol and $10 \%$ glycerol) using a French pressure cell followed by centrifugation at 20,000 x g. Fumarate reductase was assayed in $1.5 \mathrm{ml}$ glass cuvettes with $\mathrm{N}_{2}$ as gas phase at $37^{\circ} \mathrm{C}$. The $0.8 \mathrm{ml}$ standard assay mixture contained $50 \mathrm{mM}$ Tris $/ \mathrm{HCl}$ buffer $\mathrm{pH}=7.4,4 \mathrm{mM}$ dithiothreitol, $5 \mathrm{mM} \mathrm{MgCl}, 250$ $\mu \mathrm{M}$ reduced methyl viologen, $1 \mathrm{mM}$ fumarate and 50 to $400 \mu \mathrm{g}$ cell extract protein. The reactions were started by the addition of $1 \mathrm{mM}$ fumarate and the reduction of fumarate was monitored by following the decrease in absorbance at $604 \mathrm{~nm}\left(\varepsilon=26.8 \mathrm{mM}^{-1} \mathrm{~cm}^{-1}\right.$ per 2 
electron transfer). One unit (U) is the amount catalysing the reduction of $1 \mu$ mol fumarate per minute at concentrations of $250 \mu \mathrm{M}$ methyl viologen and $1 \mathrm{mM}$ fumarate

Expression of glutathione S-transferase (GST) fused to K. pneumoniae fnr in $E$. coli NCM1529.

The recombinant pRS131 containing the fnr gene of $K$. pneumoniae fused at the 5 ' end to the 3' end of the gene for GST was constructed by cloning the PCR amplified fnr into the BamHI and EcoRI restriction recognition sites of pGEX-2T (Pharmacia, Freiburg, Germany). K. pneumoniae fnr was amplified from chromosomal DNA using a set of primers with synthetic restriction recognition sites (underlined): a sense primer with an additional BamHI restriction recognition site 5 , of the start codon (5'ATATCAATGGATCCCTGAGCAGACTTATGATCC3') and an antisense primer with a EcoRI restriction recognition site downstream of the stop codcon (5'CGATCCGGCCGAATTCAGAGGGACT ATCAG3'). The PCR product was purified as described above, digested with BamHI and EcoRI and ligated into pGEX-2T, which had been linearized with the corresponding enzymes, resulting in plasmid pRS131. The PCR product cloned into pGEX-2T was sequenced, revealing no mutation of $f n r$ and correct insertion. From the sequence, the GST-Fnr fusion protein is predicted to have a molecular mass of $58 \mathrm{kDa}$ and a recognition site for thrombin between GST and Fnr. pRS131 was transformed into E. coli NCM1529, which grows well under anaerobic conditions. For expression of the GST-Fnr fusion protein, E. coli NCM1529/pRS131 was grown aerobically or anaerobically with $\mathrm{N}_{2}$ as gas phase in minimal medium (modified K-medium, Schmitz et al. 1996) with $0.8 \%$ glucose as the $\mathrm{C}$-source and $10 \mathrm{mM}$ ammonium as the nitrogen source. Expression of the fusion protein was induced with $1 \mathrm{mM}$ isopropyl-ß-D-thiogalactopyranoside (IPTG) when cultures reached an O.D. $600=0.6$. Cell extract was prepared by disruption of the cells in breakage buffer ( 50 $\mathrm{mM}$ Tris/ $\mathrm{HCl}$ buffer $\mathrm{pH}=7.6$ containing $10 \%$ glycerol) using a French pressure cell followed by centrifugation at 20,000 x g. Fusion proteins were purified from the supernatant by affinity chromatography with glutathione-Sepharose 4B (Pharmacia) according the instruction protocol of the manufacturer. In the case of anaerobic purification all steps described were performed under a nitrogen atmosphere in an anaerobic chamber and the buffers employed contained 2.0 $\mathrm{mM}$ dithiothreitol. 


\section{Determination of non-hem iron, acid-labile sulfur, and protein.}

Non-hem iron was determined colorimetrically as described by Fish (1988). Acid-labile sulfur was analyzed using methylene blue (Cline 1969). Protein was determined via the method of Bradford (1976) with the BioRad protein assay using bovine serum albumin as standard.

\section{SDS-PAGE Analyses.}

Sodium dodecyl sulfate-polyacrylamide gel electrophoresis was performed according to Laemmli using 12.8\% acrylamide (Laemmli 1970). Gels were stained for protein with Coomassie Brilliant Blue.

\section{RESULTS AND DISCUSSION}

The present work was designed to characterize the oxygen-sensing system in $K$. pneumoniae by cloning the $f n r$ homologue. We expressed the Fnr protein from K. pneumoniae in fusion to the glutathion-S transferase and analyzed purified protein for iron-sulfur clusters.

Cloning and nucleotide sequence of $\boldsymbol{K}$. pneumoniae fur. A 100-bp fragment encoding part of $K$. pneumoniae fnr was amplified by PCR using $K$. pneumoniae chromosomal DNA as template and using primers based on the N-terminal sequence of the E. coli fnr gene. This fragment was labeled with digoxigenin-dUTP and used as a hybridization probe to screen a cosmid library of $K$. pneumoniae chromosomal DNA as described in Materials and Methods. 
A)
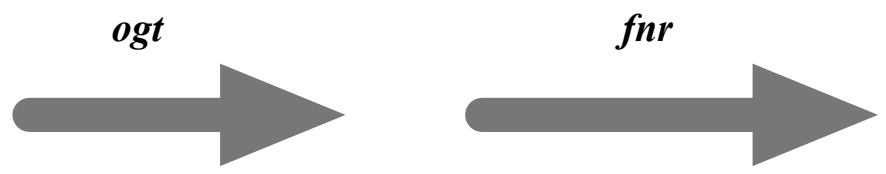

$y d a A$

EcoRI

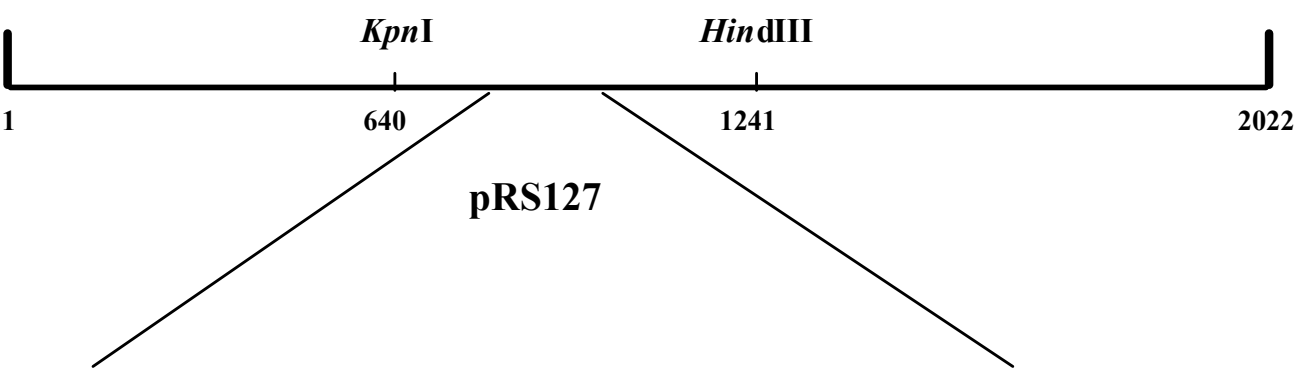

B)

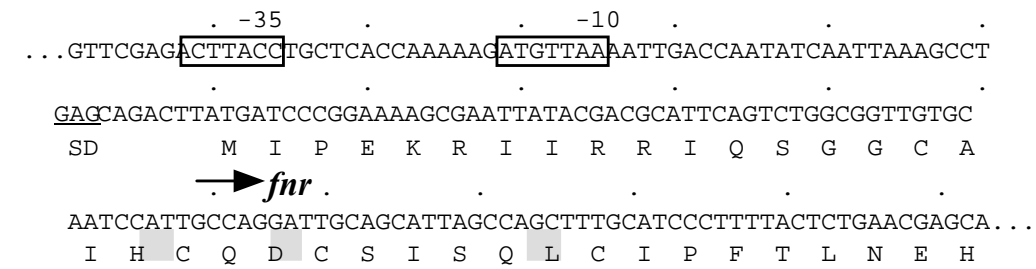

Figure 1: Organization of the cloned region of $K$. pneumoniae (A) and the sequence for the promoter and Nterminal region of $f n r$ (B). The deduced amino acid sequence is given in capital letters; the amino acid symbols (one letter code) are written below the first nucleotide of the corresponding codon. Three of the four cystein residues near the $\mathrm{N}$-terminus, which are apparently required for the [4Fe-4S)-cluster ligation, are marled in grey. A potential ribosome binding site (SD) is underlined and a putative s70-dependent promoter sequence is boxed. The sequence of the cloned region has been submitted to GenBank under accession number AF220669.

K. pneumoniae fnr was identified on a $2.1 \mathrm{kbp} E c o \mathrm{RI} / B a m \mathrm{HI}$ fragment. This fragment was subcloned into $\mathrm{pSK}^{+}$Bluescript and the resulting plasmid designated pRS127. The insert of pRS127 was entirely sequenced in both directions. Analysis of the sequence revealed two open reading frames, $\operatorname{orf} A$ and $\operatorname{orf} B$, and part of a third putative open reading frame ( $\left.\operatorname{orfC}^{\prime}\right)$ as shown in Fig. 1. $\operatorname{orf} B$ showed high similarities to $f n r$ from $E$. coli and was therefore designated as fnr. The open reading frame upstream of $f n r$ was identified as $o g t$ by homology to the equivalent $E$. coli gene and $o r f C^{\prime}$ downstream of $f n r$ shows homology to $y d a A^{\prime}$ of $E$. coli. The $f n r$ gene of $K$. pneumoniae is preceded by a weak ribosomal binding site, appropriately spaced from the start codon; in addition, a sequence for a putative $\sigma^{70}$-dependent promoter is located upstream of $f n r$ in position -61 to -32 (Fig. 1). The $f n r$ gene (753 bp) codes for a polypeptide of 250 amino acids with a predicted molecular mass of $27939 \mathrm{Da}$, which shows $98 \%$ amino acid identity to Fnr of E. coli (Shaw \& Guest 1982). In addition Fnr of K. pneumoniae (Fnr ${ }_{\mathrm{Kp}}$ ) contained all four essential cysteine residues (Cys20, Cys22, Cys29 and Cys122) which are presumed to comprise the oxygen-sensing $[4 \mathrm{Fe} 4 \mathrm{~S}]^{2+}$-center in E. coli $\mathrm{Fnr}\left(\mathrm{Fnr}_{\mathrm{Ec}}\right)$ (Spiro \& Guest 1988). 


\section{Function of $K$. pneumoniae Fnr as an oxygen-sensitive transcriptional regulator.}

Based on high similarity, K. pneumoniae $\mathrm{Fnr}\left(\mathrm{Fnr}_{\mathrm{Kp}}\right)$ is presumed to function as a transcriptional activator of nitrate metabolism under anaerobic conditions in the same manner as E. coli Fnr. We therefore studied growth on glycerol and nitrate under anaerobic conditions of an E. coli strain with a chromosomal fnr deletion (RAS1). This mutant strain is not able to grow on nitrate and glycerol in the absence of oxygen (Fig. 2). A plasmid-bound copy of the fnr gene of K. pneumoniae under the control of the tetracycline resistance promoter (pRS137), was able to completely complement the mutation, and allow growth on glycerol and nitrate (RAS5) as it is the case for a plasmid born copy of the native fnr gene of E. coli (pRS120) (see Fig. 2).

To obtain additional evidence we studied expression of another Fnr-dependent gene in E. coli strains with a chromosomal $f n r$ deletion. Synthesis of fumarate reductase under anaerobic growth conditions was determined by measuring the activity of fumarate reductase in a fnr deletion strain and the same strain containing K. pneumoniae fnr on a plasmid.

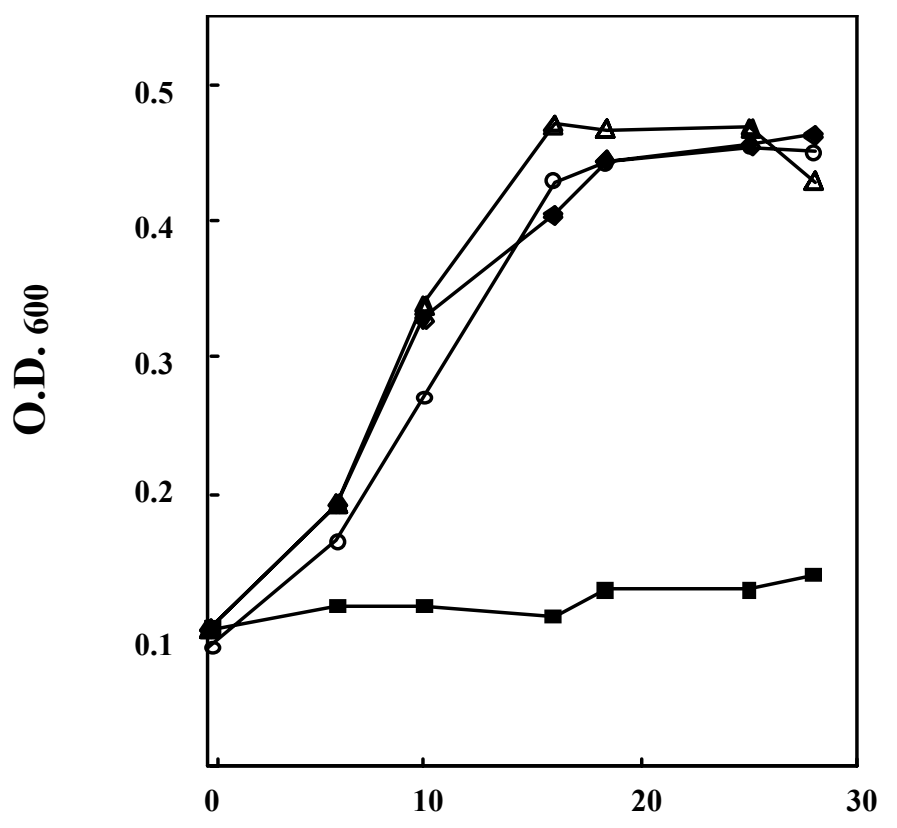

Time (h)

Figure 2: Growth of E. coli under anaerobic conditions in minimal medium supplemented with $0.8 \%$ glycerol as the sole $\mathrm{C}$-source and $1 \% \mathrm{KNO}$ as the sole nitrogen source (see Materials and methods). (closed diamonds), NCM1529 (parental strain); (closed squares), RAS1 (NCM1529 but fnr::Tn10); (open circles), RAS1 transformed with pRS120 (E.coli fnr controlled by the tet promoter); (open triangles), RAS1 transformed with pRS137 (K. pneumoniae fnr controlled by the tet promoter). 
Fumarate reductase activity in the $f n r$ deletion strain (RAS1) was determined to be $23 \mathrm{mU} /$ $\mathrm{mg}$ cell extract protein, which was equivalent to $10 \%$ of the activity in the parental strain NCM1529 (231 mU / mg cell extract protein). When the fnr gene of K. pneumoniae was expressed in trans under the control of the tet promoter (RAS5) it was able to complement the fnr mutation in E. coli and allow significant higher fumarate reductase activity under anaerobic conditions (174.5 mU / mg cell extract protein). These results indicate that $\mathrm{Fnr}_{\mathrm{Kp}}$ is functional in $E$. coli and apparently acts as an oxygen-sensing transcriptional regulator.

Purification and characterization of heterologous expressed Fnr $_{\mathrm{Kp}}$. In order to characterize the iron-sulfur clusters we fused $\mathrm{Fnr}_{\mathrm{Kp}}$ to the glutathione S-transferase (GST) by cloning the $f n r$ gene into pGEX-2T (see Materials and Methods). The resulting plasmid, which contains the gst-gene $C$-terminally fused to $K$. pneumoniae fnr under the control of the tac promoter, was designated pRS131. After transforming pRS131 into E. coli NCM1529, which grows well under anaerobic conditions (He et al. 1997), the fusion protein was synthesized in minimal medium under aerobic and anaerobic growth conditions as described in Materials and Methods with ammonium as nitrogen source. Under both growth conditions, induction of the fusion protein at an optical density of O.D. $600=0.6$ resulted in a retarded growth. The overexpressed fusion protein fractions were purified in the presence and absence of molecular oxygen, respectively, by Glutathione-Sepharose 4B affinity chromatography and cleaved with the site-specific protease thrombin. In both cases, homogeneous Fnr $_{\mathrm{Kp}}$ preparations were obtained, as revealed by sodium dodecylsulfate/polyacrylamide gel electrophoresis. The apparent molecular mass of $\mathrm{Fnr}_{\mathrm{Kp}}$ was determined to be $28 \mathrm{kDa}$ (see Fig. 3). After purification, the cofactors of both protein fractions were determined. The aerobic Fnr preparations were found to contain less than $0.1 \mathrm{~mol}$ of acid-labile sulfur and $1.0 \mathrm{~mol}$ iron per mol Fnr. For the anaerobic Fnr preparations, $2.6 \mathrm{~mol}$ iron and $2.2 \mathrm{~mol}$ acid-labile sulfur was found per mol Fnr, indicating the presence of an $[3 \mathrm{Fe} 3 \mathrm{~S}]$-cluster or an $[4 \mathrm{Fe} 4 \mathrm{~S}]$-cluster in the anaerobic protein. Synthesis and purification under aerobic conditions apparently resulted in the disruption of the iron-sulfur cluster and loss of the iron. These results indicate that $\mathrm{Fnr}_{\mathrm{Kp}}$ apparently contains an iron-sulfur center responsible for oxygen sensing, as it is the case for $\mathrm{Fnr}_{\mathrm{Ec}}$, which is disrupted in the presence of molecular oxygen (Green et al. 1996, Khoroshilova et al. 1997, Kiley \& Beinert 1998). 


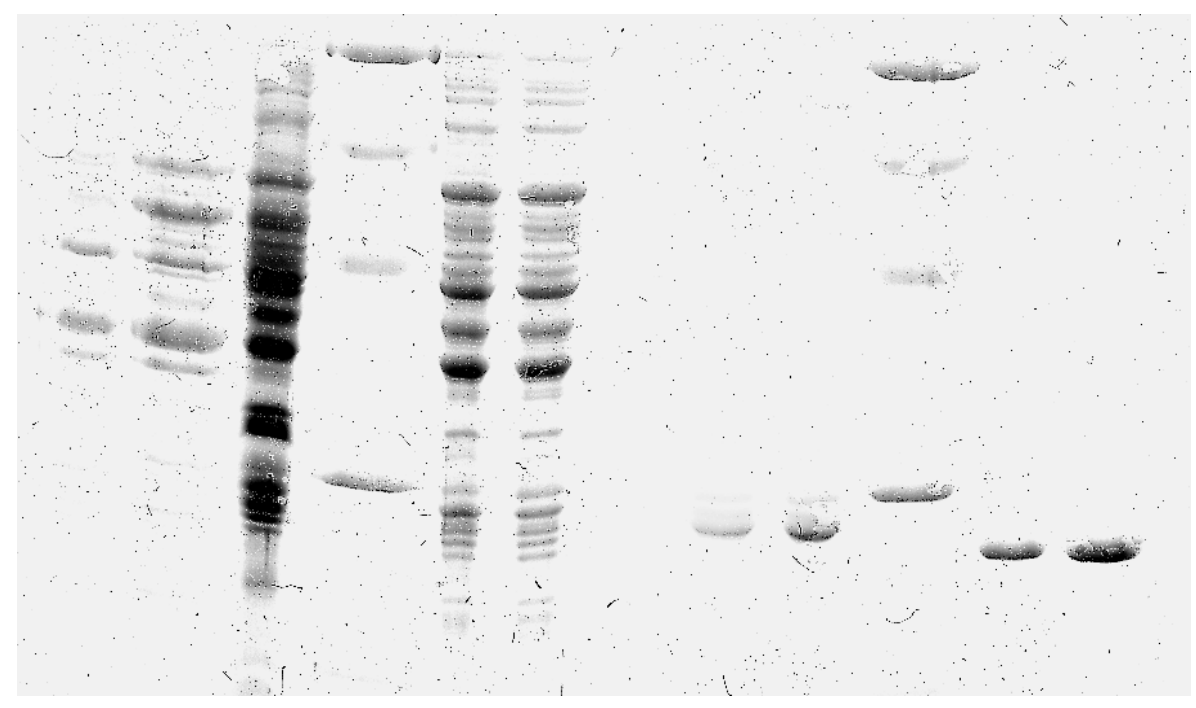

Figure 3: Purification of $K$. pneumoniae Fnr fused to glutathion S-transferase (GST) and synthesized under anaerobic conditions. Various stages in the purification are seperated by SDS-Page (12.8\%). Lanes: 1 and 2 , whole cell extract before and after IPTG induction, respectively; 3, low-speed supernatant from cell extract; 4 and 10. low molecular mass marker (Pharmacia); 5, 6 and 7, wash fractions of GST-Fnr bound to Glutathione Sepharose 4B; 8 and 9, flow through fractions following thrombin digest of GST-Fnr bound to Glutathione Sepharose 4B; 11 and 12, GST fraction eluted with glutathion supplemented buffer. The gel was stained with coomassie Brillant Blue R250.

In order to further analyse the iron-sulfur center of $\mathrm{Fnr}_{\mathrm{Kp}}$ we studied the spectroscopic properties of the anaerobic $\mathrm{Fnr}_{\mathrm{Kp}}$ protein fraction. (i) UV-visible spectroscopy of the anaerobic

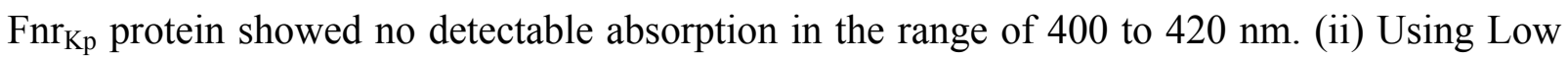
Temperature EPR analyses, we revealed no signal typical for an iron sulfur cluster for the anaerobic Fnr fraction (data not shown). This might be due to the low protein concentrations we observed from the anaerobic protein purification (approximately $0.5 \mathrm{mg} / \mathrm{ml}$ ) or due to disruption of the iron-sulfur clusters during the purification procedure even when performed under anaerobic conditions.

In summary. In order to characterize the oxygen-sensing system in K. pneumoniae we have cloned and characterized the fnr gene of $K$. pneumoniae. Analyses of the $K$. pneumoniae fnr gene showed high similarities to the E. coli fnr gene (98\% amino acid identity, Shaw \& Guest 1982). The ability of $f n r_{\mathrm{Kp}}$ to functionally complement $f n r_{\mathrm{Ec}}$ was shown in vivo by restoration of growth on glycerol plus nitrate, and expression of Fnr-dependent genes ( $f r d \mathrm{ABCD})$ in an E. coli fnr deletion strain transformed with a plasmid-bound copy of $\mathrm{Fnr}_{\mathrm{Kp}}$. 
These results indicate that $\mathrm{Fnr}_{\mathrm{Kp}}$ activates transcription of genes in a similar way like E. coli Fnr. They further suggest a similarity in the oxygen-sensing mechanism of the two organisms. In addition, characterization of purified protein indicated the presence of an oxygen sensitive $[4 \mathrm{Fe} 4 \mathrm{~S}]^{2+}$-center in $\mathrm{Fnr}_{\mathrm{Kp}}$ : (i) The deduced amino acid sequence of $K$. pneumoniae fnr contained all four essential cysteine residues near the N-terminus, which are required for the oxygen-sensing function (Spiro \& Guest 1988, Khoroshilova et al. 1997). (ii) Determination of iron and acid-labile sulfur in aerobic- and anaerobic-purified protein fractions suggested the presence of an iron-sulfur cluster, which is apparently disrupted upon the influence of oxygen. 


\title{
Chapter 3:
}

\section{Fnr is required for NifL-dependent oxygen control of nif gene expression in Klebsiella pneumoniae}

\begin{abstract}
In Klebsiella pneumoniae, NifA dependent transcription of nitrogen fixation (nif) genes is inhibited by NifL in response to molecular oxygen and combined nitrogen. We recently showed that K. pneumoniae NifL is a flavoprotein, which apparently senses oxygen through a redox-sensitive, conformational change. We have now studied the oxygen regulation of NifL activity in Escherichia coli and K. pneumoniae strains by monitoring its inhibition of NifAmediated expression of $K$. pneumoniae $\phi($ nifH'-'lacZ $)$ fusions in different genetic backgrounds. Strains of both organisms carrying fnr null mutations failed to release NifL inhibition of NifA transcriptional activity under oxygen limitation: nif induction was similar to the induction under aerobic conditions. When the transcriptional regulator Fnr was synthesized from a plasmid, it was able to complement, i.e., to relieve NifL inhibition in the fnr-backgrounds. Hence, Fnr appears to be involved, directly or indirectly, in NifL-dependent oxygen regulation of nif gene expression in K. pneumoniae. The data indicate that in the absence of Fnr NifL apparently does not receive the signal for anaerobiosis. We therefore hypothesize that in the absence of oxygen, Fnr, as the primary oxygen sensor, activates transcription of a gene(s) whose product(s) function to relieve NifL inhibition by reducing the FAD cofactor under oxygen-limiting conditions.
\end{abstract}




\section{Introduction}

In diazotrophic proteobacteria, transcription of the nitrogen fixation (nif) genes is mediated by the nif-specific activator protein NifA, a member of a family of activators that functions with $\sigma^{54}$ (Dixon, 1998, Fischer, 1994). Both the expression and the activity of NifA can be regulated in response to the oxygen and / or combined nitrogen status of the cells; the mechanisms of the regulation differ with the organism. In Klebsiella pneumoniae and Azotobacter vinelandii, NifA transcriptional activity is regulated by a second regulatory protein, NifL. This negative regulator of the nif genes inhibits the transcriptional activation by NifA in response to combined nitrogen and or external molecular oxygen. The translationally-coupled synthesis of the two regulatory proteins, immunological studies, complex analyses and studies using the two-hybrid system in Saccharomyces cerivisiae imply that the inhibition of NifA activity by NifL apparently occurs via direct protein-protein interaction (Govantes et al., 1998, Henderson et al., 1989; Lei et al., 1999; Money et al., 1999). The mechanism by which nitrogen is sensed in K. pneumoniae and A. vinelandii is currently the subject of extensive studies. Very recently, He et al. (He et al., 1998), and Jack et al. (1999) provided evidence that in K. pneumoniae, the second PII protein, GlnK, is required for relief of NifL inhibition under nitrogen-limiting conditions. This indicates that GlnK regulates NifL inhibition of NifA in response to the nitrogen status of the cells by interacting with NifL or NifA.

In both organisms, $K$. pneumoniae and $A$. vinelandii, the negative regulator NifL is a flavoprotein with an N-terminally bound flavin adenine dinucleotide as a prosthetic group (Hill et al., 1996; Klopprogge and Schmitz, 1999: Schmitz, 1997). In vitro, the oxidized form of NifL inhibits NifA activity, whereas reduction of the FAD cofactor relieves NifL inhibition (Hill et al., 1996; Macheroux et al., 1999). This indicates that NifL apparently acts as a redox switch in response to the environmental oxygen status and allows NifA activity, only under oxygen-limiting conditions. We recently showed that in vivo, the presence of iron is required to relieve inhibitory effects of NifL on transcriptional activation by NifA and, additionally, that iron is not present in NifL (Schmitz, 1997; Schmitz et al., 1996). Therefore, we have postulated that an unidentified iron-containing protein may be the physiological reductant for 
NifL. This putative iron-containing protein is apparently not nif specific since NifL function is regulated normally in response to cellular nitrogen and oxygen availability in Escherichia coli in the absence of nif proteins other than NifA (He et al., 1998).

The key question concerning the oxygen signal transduction in K. pneumoniae is, whether NifL senses oxygen directly via a redox-induced conformational change, or whether oxygen is detected by a more general oxygen-sensing system, which then regulates NifL by inducing the oxidation or reduction of the flavin cofactor. One candidate for a general oxygen sensor is the transcriptional fumarate nitrate regulator (Fnr) (Spiro, 1994; Spiro and Guest, 1990), which in the case of E. coli Fnr, senses oxygen via an oxygen-labile iron-sulfur $\left([4 \mathrm{Fe}-4 \mathrm{~S}]^{+2}\right)$-cluster and is involved in signal transduction of the cellular redox state (Green et al., 1996; Khoroshilova et al., 1997; Melville and Gunsalus, 1990; Unden and Schirawski, 1997). Recently we cloned and sequenced the fnr gene of $K$. pneumoniae and characterized the protein (Grabbe et al., 2000). As the K. pneumoniae Fnr amino acid sequence is $98 \%$ identical to the E. coli Fnr and contains an iron-sulfur cluster, we have now tested the hypothesis that Fnr transduces the oxygen signal to NifL. We present evidence that in the absence of Fnr, NifL inhibits NifA activity under oxygen-limitation, suggesting that Fnr is required for relief of NifL inhibition in $K$. pneumoniae under anaerobic conditions.

\section{Materials and Methods}

Bacterial strains and plasmids. The bacterial strains and plasmids used in this work are listed in Table 2. Plasmid DNA was transformed into E. coli cells according to the method of Inoue et al. (1990) and into K. pneumoniae cells by electroporation. Transduction by phage P1 was performed as described previously (Silhavy et al., 1984).

E. coli strains. E. coli NCM1529, which contains a $\varnothing($ nifH'-'lacZ) fusion (He et al. 1997), and derivatives of NCM1529 were chosen to study NifA/NifL regulation in E. coli. The fnr::Tn10 allele was transferred from the fnr::Tn10 derivative of M182 (Jayaraman et al., 1988) into NCM1529 by P1-mediated transduction with selection for tetracycline resistance, 
resulting in RAS1 (Grabbe et al., 2001a). Strains RAS6, RAS7, RAS8, RAS9, RAS10, RAS11 and RAS12 contain plasmids pRS107, pNH3, pJES851, pNH3 plus pRS79, pNH3 plus pRS120, pNH3 plus pMCL210, and pNH3 plus pACYC184, respectively, in RAS1. To construct an independent second $f n r$ null mutant, the [Kan $\left.{ }^{\mathrm{r}}-\left(n i f H^{\prime}-' l a c Z\right)\right]$ allele was transferred from strain NCM1529 by P1-mediated transduction into the independent fnr mutant strain RM101 (Sawers and Suppman, 1992) and into the parental strain MC4100 with selection for kanamycin resistance, resulting in RAS13 and RAS21, respectively. Strains RAS25, RAS14, RAS15, RAS16 and RAS17 contain plasmids pRS107, pNH3, pJES851, pNH3 plus pRS120 and pNH3 plus pACYC184, respectively in RAS13.

Klebsiella strains. K. pneumoniae strains M5al (wild type) and UN4495 ( $\varnothing($ nifK-lacZ)5935 Dlac-4001 his D4226 Gal' ${ }^{\mathrm{r}}$ (McNeil et al., 1981) were provided by Gary Roberts.

Construction of a fnr:: $\Omega$ mutation: Strain RAS18 was obtained by insertion of a kanamycin resistance cassette (Prentki et al., 1984) into the fnr gene of $K$. pneumoniae UN4495 as achieved in the following steps. (i) The $2.1 \mathrm{kbp} E c o \mathrm{RI} /$ BamHI fragment, which carries the ogt-fnr-ydaA'- region of $K$. pneumoniae, was subcloned into pBluescript $\mathrm{SK}^{+}$to produce pRS127. (ii) A $2.1 \mathrm{~kb}$ HindIII cassette containing an $\Omega$ interposon fragment with a kanamycin

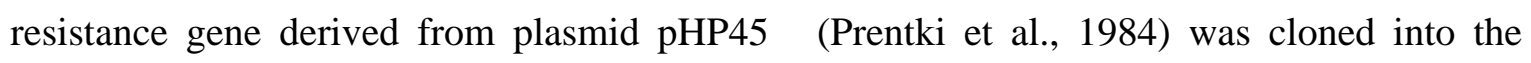
HindIII site of fnr in pRS127 to yield plasmid pRS142. (iii) A $2.9 \mathrm{~kb}$ PCR fragment carrying $f n r:: \Omega$ was generated using pRS142 as template and a set of primers which were homologue to the fnr flanking 5'- and 3'-regions with additional BamHI synthetic restriction recognition sites (underlined) (5'ATATCAATGGATCCCTGAGCAGACTTA TGATCC3', sense primer; 5'CTTATATGGATCCAATGAAACAGGGGAGGA3', antisense primer). The $2.9 \mathrm{~kb}$ PCR product was cloned into the BamHI site of the $s a c B$-containing vector pKNG101 (18), creating plasmid pRS144. The correct insertion was analyzed by sequencing. (iv) pRS144 was transformed into K. pneumoniae UN4495 and recombinant strains (generated by means of a double cross over) were identified by the ability to grow on LB supplemented with 5\% 
sucrose and resistance to kanamycin. The $f n r:: \Omega$ mutation in strain RAS18 was confirmed by southern blot analysis (Sambrook et al., 1989) and by PCR.

Strains RAS26 and RAS28 contain pRS159 and pJES839, respectively, in K. pneumoniae UN4495 and strains RAS19, RAS27 and RAS29 contain pRS137, pRS159 and pJES839, respectively, in RAS18.

Table 2: Bacterial strains and Plasmids used in this study.

\begin{tabular}{|c|c|c|}
\hline$\underline{\text { Strains / plasmids }}$ & $\underline{\text { Relevant genotype and/or characteristic(s) }}$ & $\underline{\text { Reference or description }}$ \\
\hline E. coli strains & & \\
\hline NCM1529 & $\begin{array}{c}\text { araD1394(argF-lacU)169 fth D5301 } \\
\text { gyrA219 non-9 rpsL150 ptsF25 relA1 deoCl } \\
\text { trpDC700putPA1303::[Kan'-(nifH'-'lacZ)] } \\
\text { (wild type) }\end{array}$ & He et al. 1997 \\
\hline NCM1528 & NCM1529/pNH3 & He et al. 1997 \\
\hline NCM1527 & NCM1529/pJES851 & He et al. 1997 \\
\hline RAS1 & NCM1529 but fnr::Tn10 & Grabbe et al. 2001a \\
\hline RAS2 & NCM1529/pRS107 & This study \\
\hline RAS6 & RAS1/pRS107 & This study \\
\hline RAS7 & RAS1/pNH3 & This study \\
\hline RAS8 & RAS1/pJES851 & This study \\
\hline RAS9 & RAS1/pNH3 and pRS79 & This study \\
\hline RAS10 & RAS1/pNH3 and pRS120 & This study \\
\hline
\end{tabular}




\begin{tabular}{|c|c|c|}
\hline RAS11 & RAS1/pNH3 and pMCL210 & This study \\
\hline RAS12 & RAS1/pNH3 and pACYC184 & This study \\
\hline RM101 & MC4100 but $\Delta f n r$ & Schmitz 1997 \\
\hline RAS13 & RM101 but [Kan'-(nifH'-'lacZ)] & This study \\
\hline RAS21 & MC4100 but [Kan ${ }^{\mathrm{r}}-($ nifH'-' lacZ)] & This study \\
\hline RAS22 & $\mathrm{RAS} 21 / \mathrm{pNH} 3$ & This study \\
\hline RAS23 & RAS21/pJES851 & This study \\
\hline RAS24 & RAS21/pRS107 & This study \\
\hline RAS14 & RAS13/pNH3 & This study \\
\hline RAS15 & RAS13/pJES851 & This study \\
\hline RAS25 & RAS13/pRS107 & This study \\
\hline RAS16 & RAS13/pNH3 and pRS120 & This study \\
\hline RAS17 & RAS13/pNH3 and pACYC184 & This study \\
\hline$\frac{\underline{\text { K. pneumoniae }}}{\underline{\text { strains }}}$ & & \\
\hline M5al & Wild type & \\
\hline UN4495 & $\begin{array}{c}\emptyset(\text { nifK-lacZ)5935 } \Delta l a c-4001 \text { his D4226 } \\
\text { Gal }^{\mathrm{r}}\end{array}$ & MacNeil et al. 1981 \\
\hline RAS18 & $\begin{array}{c}\varnothing(\text { nifK-lacZ)5935 } \Delta l a c-4001 \text { his D4226 } \\
\text { Gal }^{\mathrm{r}} \text { fnr:: } \Omega\end{array}$ & This study \\
\hline
\end{tabular}




\begin{tabular}{|c|c|c|}
\hline RAS19 & RAS18/pRS137 & This study \\
\hline RAS20 & RAS18/pACYC184 & This study \\
\hline RAS26 & UN4495/pRS159 & This study \\
\hline RAS27 & RAS18/pRS159 & This study \\
\hline RAS28 & UN4495/pJES839 & He et al. 1997 \\
\hline RAS29 & RAS18/pJES839 & This study \\
\hline RAS30 & $\begin{array}{l}\mathrm{UN} 4495 \Delta(\text { nifLA }) 6293:: \mathrm{Km} / \\
\text { pJES839 }\end{array}$ & $\begin{array}{l}\text { Schmitz et al. } 1996 \text { and } \\
\text { this study }\end{array}$ \\
\hline Plasmids & & \\
\hline pNH3 & $\begin{array}{l}\text { K. pneumoniae nifLA controlled by the tac } \\
\text { promoter }\end{array}$ & Henderson et al. 1989 \\
\hline pJES839 & $\begin{array}{c}\text { pNH3 but additional tetracycline resistance } \\
\text { cassette }\end{array}$ & Schmitz et al. 1996 \\
\hline pJES851 & $\begin{array}{l}\text { K. pneumoniae nifA controlled by the tac } \\
\text { promoter }\end{array}$ & Schmitz et al. 1996 \\
\hline pRS79 & $\begin{array}{l}\text { E. coli fnr controlled by the } l a c \text { promoter on } \\
\text { pMCL } 210\end{array}$ & This study \\
\hline pRS107 & $\begin{array}{c}\text { K. pneumoniae nif } L^{\mathrm{C} 184 \mathrm{~S} / \mathrm{C} 187 \mathrm{~s}} \text { nifA controlled } \mathrm{b} \\
\text { the tac promoter }\end{array}$ & This study \\
\hline pRS159 & $\begin{array}{c}\text { K. pneumoniae nifL }{ }^{\mathrm{C} 184 \mathrm{SC} / 187 \mathrm{~s}} \text { nifA controlled } \mathrm{b} \\
\text { the tac promoter }\end{array}$ & This study \\
\hline pRS120 & $\begin{array}{l}\text { E. coli fnr controlled by the tet promoter on } \\
\text { pACYC184 }\end{array}$ & Grabbe et al. 2001a \\
\hline
\end{tabular}




\begin{tabular}{|c|c|c|}
\hline pRS127 & $\begin{array}{l}2.1 \text { kbp fragment in pBluescript } \mathrm{SK}^{+} \\
\text {containing K. pneumoniae fnr }\end{array}$ & Grabbe et al. 2001a \\
\hline pRS137 & $\begin{array}{l}\text { K. pneumoniae fnr controlled by thetet } \\
\text { promoter on pACYC184 }\end{array}$ & Grabbe et al. 2001a \\
\hline pACYC184 & Low copy vector & $\begin{array}{c}\text { New England Biolabs, } \\
\text { UK }\end{array}$ \\
\hline pMCL210 & Low copy vector & Nakano et al. 1995 \\
\hline $\begin{array}{c}\text { pBluescript } \\
\mathrm{SK}^{+}\end{array}$ & Cloning vector & Stratagene, La Jolla, US \\
\hline
\end{tabular}

Construction of plasmids. Plasmid pRS107 contains the K. pneumoniae nifL $L^{\mathrm{C} 1845 / \mathrm{C} 187 \mathrm{~s}}$ nifAoperon under the control of the tac promoter, in which the Cys ${ }^{184}$ and $\mathrm{Cys}^{187}$ of nifL are changed to serine $\left(\operatorname{Ser}^{184}\right.$-Ala-Asp-Ser ${ }^{187}$ ). It was constructed from pNH3 (Henderson et al., 1989) by introducing the double mutation into nifL by site directed mutagenesis. Site directed mutagenesis was performed using the GeneEditor System (Promega) according to the protocol of the manufacturer. The double mutation was confirmed by sequencing. Plasmid pRS159 was constructed by inserting a tetracycline-resistance cassette (Schmitz et al., 1996) into the ScaI site of plasmid pRS107. Plasmid pRS79 contains the E. coli fnr gene inserted into the BamHI and PstI site of pMCL210 (Nakano et al., 1995) under the control of the lac promoter. pRS120 and pRS137 contain E. coli fnr gene and K. pneumoniae fnr gene, respectively, inserted into the SalI and BamHI site of pACYC184 and thereby expressed from the tet promoter (Grabbe et al., 2001a).

Growth. K. pneumoniae and E. coli strains were grown under anaerobic conditions with $\mathrm{N}_{2}$ as gas phase at $30^{\circ} \mathrm{C}$ in minimal medium (Schmitz et al., 1996) supplemented with $4 \mathrm{mM}$ glutamine, $10 \mathrm{mM} \mathrm{Na} \mathrm{CO}_{3}, 0.3 \mathrm{mM}$ sulfide and $0.002 \%$ resazurine to monitor anaerobiosis. The medium was further supplemented with $0.004 \%$ histidine and with $0.4 \%$ sucrose as sole 
carbon source for K. pneumoniae strains. For E. coli strains, the medium was supplemented with $0.1 \mathrm{mM}$ tryptophane and $0.8 \%$ glucose as the carbon source. Precultures were grown overnight in closed bottles with $\mathrm{N}_{2}$ as gas phase, in medium lacking sulfide and resazurine but supplemented with $4 \mathrm{mM}$ ammonium acetate in addition to glutamine; both ammonium and glutamine were completely utilized during growth of precultures. The cultures $(25 \mathrm{ml})$ were grown in closed bottles with $\mathrm{N}_{2}$ as gas phase at $30^{\circ} \mathrm{C}$ under strictly anaerobic conditions without shaking. Samples for monitoring growth at $600 \mathrm{~nm}$ and determining ß-galactosidase activity were taken anaerobically. In E. coli strains carrying a plasmid encoding NifL and NifA (pNH3 (12)), NifL ${ }^{\mathrm{C} 184 \mathrm{~S} / \mathrm{C} 187 \mathrm{~S}}$ and NifA (pRS107) or a plasmid encoding NifA alone (pJES851 (Schmitz et al., 1996)) expression of nifLA, nifLC184SC/187SnifA or nifA was induced from the tac promoter with $10 \mu \mathrm{M}$ IPTG (isopropyl-ß-D-thiogalactopyranoside).

Fnr phenotypes of RAS1, RAS13, RAS18 and the respective complemented strains RAS9, RAS10, RAS16 and RAS19 were tested anaerobically using glycerol and nitrate $(0.5 \%)$ as sole carbon and nitrogen source in minimal medium.

B-Galactosidase assay. NifA-mediated activation of transcription from the nifHDK promoter in K. pneumoniae UN4495 and E. coli strains was monitored by measuring the differential rate of B-galactosidase synthesis during exponential growth (units per milliliter per $\mathrm{OD}_{600}$ ) (Schmitz et al., 1996). Inhibitory effects of NifL on NifA activity were assessed by virtue of a decrease in nifH expression.

Western blot analysis. Cells were grown anaerobically in minimal medium with glutamine as nitrogen source, when the culture reached a turbidity of 0.4 to 0.7 at $660 \mathrm{~nm}, 1 \mathrm{ml}$ samples of the exponentially growing cultures were harvested and concentrated 20-fold into sodium dodecyl sulfate (SDS) gel-loading buffer (Laemmli, 1970). Samples were separated by SDS/polyacrylamide (12\%) gel electrophoresis and transferred to nitrocellulose membranes as described previously (Sambrook et al., 1989). Membranes were exposed to polyclonal rabbit antisera directed against the NifL or NifA proteins of $K$. pneumoniae, protein bands were 
detected with secondary antibodies directed against rabbit immunoglobulin $\mathrm{G}$ and coupled to horseradish peroxidase (BioRad Laboratories). Purified NifA and NifL from K. pneumoniae and prestained protein markers (New England Biolabs, UK) were used as standards.

Data deposition. $K$. pneumoniae fnr sequence has been submitted to GenBank under accession number AF220669.

\section{Results}

We recently showed that in vivo iron is specifically required for nif-induction in K. pneumoniae, and additionally, that iron is not present in NifL (Schmitz, 1997; Schmitz et al., 1996). In order to examine whether oxygen is detected by a more general system rather than by NifL directly we chose to examine the possible influence of Fnr on the nif-induction in a heterologous E. coli system. We performed all experiments under nitrogen limitinggrowth conditions to exclude NifA inhibition by NifL in response of ammonium presence. If Fnr is indeed the primary oxygen sensor, which transduces the oxygen signal to NifL, the iron requirement for the nif-induction under oxygen-limiting conditions may be based on the iron requirement for the assembly of iron sulfur clusters of Fnr.

Studying the effect of Fnr on the nif-induction in a heterologous E. coli system. In order to study the effect of Fnr on nif regulation in response to oxygen we chose a heterologous E. coli system. Strain NCM1529 carrying a chromosomal nifH'-'lacZ fusion was used as parental strain (He et al., 1997). NifL and NifA were induced independent of the Ntr system from plasmids which carried the K. pneumoniae nifLA (pNH3) and nifA (pJES851) genes under the control of the tac promoter. The two regulatory proteins were induced with $10 \mu \mathrm{M}$ IPTG to levels at which NifL function is regulated normally in response to oxygen and combined nitrogen in E. coli in the absence of nif proteins other than NifA (He et al., 1997). To study the effect of an fnr null mutation on the regulation of NifL activity in response to oxygen, an fnr null allele (fnr::Tn10) was introduced by P1 transduction into the parental 
strain NCM1529 carrying the $\varnothing($ nifH'-'lacZ) fusion as described in Materials and Methods, resulting in strain RAS1. After introducing nifLA and nifA on plasmids, the resulting strains were generally grown in mineral medium with glucose as sole carbon source and under nitrogen-limitation to exclude NifA inhibition by NifL in response to combined nitrogen. Determining the doubling times of the different strains under anaerobic and aerobic conditions revealed no significant difference in growth rates for $f n r^{-}$strains compared to the respective parental strains (Table 3). NifA-mediated activation of transcription from the nif $H^{\prime}$ promoter in the different backgrounds was monitored by determining the differential rate of $\beta$ galactosidase synthesis during exponential growth. Inhibitory effects of NifL on NifA activity in strain RAS7 carrying the fnr null allele and carrying nifLA on a plasmid are detectable, they result in a decrease in nifH-expression. Interestingly, under oxygen-limiting conditions strain RAS7 showed a $\beta$-galactosidase synthesis rate from the nif $H^{\prime}$-promoter of only $100 \pm 10 \mathrm{U} / \mathrm{ml}$ $\mathrm{OD}_{600}$ when nifLA was induced with $10 \mu \mathrm{M}$ IPTG. This is in the range of synthesis rate under aerobic conditions in the parental strain NCM1528 (60 $\left.\pm 5 \mathrm{U} / \mathrm{ml} \mathrm{OD}_{600}\right)$ and equivalent to $3 \%$ of the synthesis rate under anaerobic conditions in NCM1528 $\left(3000 \pm 100 \mathrm{U} / \mathrm{ml} \mathrm{OD}_{600}\right)$ (Table 3).

Table 3: Effects of an $f n r$ null allele on activity of the $K$. pneumoniae NifL protein in different $E$. coli backgrounds.

\begin{tabular}{|c|c|c|c|c|}
\hline Strain & Relevant genotype & $\begin{array}{c}\text { Presence } \\
\text { of }\end{array}$ & $\begin{array}{l}\text { Expression of } \\
\text { nifH'-'lacZ' }\end{array}$ & $\begin{array}{c}\text { Doubling } \\
\text { time }\end{array}$ \\
\hline & & oxygen & $(\mathrm{U} / \mathrm{ml} \cdot \text { O.D.600 })^{\mathrm{a}}$ & (h) \\
\hline NCM1528 & Wild type/Ptac-nifLA & - & $3000 \pm 100$ & 5.0 \\
\hline NCM1528 & Wild type/Ptac-nifLA & + & $60 \pm 5$ & 2.0 \\
\hline NCM1527 & Wild type/Ptac-nifA & - & $5300 \pm 200$ & 4.8 \\
\hline
\end{tabular}


NCM1527 Wild type/Ptac-nifA

$5118^{d}$

RAS2 Wild type/Ptac-nifL' nifA

$2950 \pm 120$

RAS2 Wild type/Ptac-nifL- nifA

$2900 \pm 50$

RAS8 $^{\mathrm{b}} \quad$ fnr ${ }^{-} /$Ptac-nifA

$4800 \pm 100$

RAS8 $^{\mathrm{b}} \quad$ fnr ${ }^{\circ}$ Ptac-nifA

$+\quad 5200 \pm 200$

RAS6 $^{\text {b }} \quad$ fnr/Ptac-nifL ${ }^{-}$nifA

$-\quad 2800 \pm 100$

RAS6 $^{\text {b }} \quad$ fnr ${ }^{-}$Ptac-nifL $L^{-}$nifA

$+\quad 3000 \pm 200$

RAS7 $^{\text {b }} \quad$ fnr/Ptac-nifLA

$100 \pm 10$

RAS7 $^{\text {b }} \quad$ fnr/Ptac-nifLA

$30 \pm 3$

RAS9 $^{\text {b }} \quad$ fnr/Ptac-nifLA/Plac fnr

$3000 \pm 100$

RAS10 $^{\mathrm{b}} \quad$ fnr $/$ Ptac-nifLA/Ptet fnr

$2870 \pm 70$

fnr ${ }^{-}$Ptac-

RAS11 ${ }^{\text {b }}$

$$
66 \pm 5
$$

nifLA/pMCL210

fnr/Ptac-

RAS12 ${ }^{b}$

nifLA/pACYC184

$$
70 \pm 6
$$

RAS22 Wild type/Ptac-nifLA

$$
3500 \pm 80
$$

RAS22 Wild type/Ptac-nifLA

$70 \pm 5$

RAS23 Wild type/Ptac-nifA

$5900 \pm 250$

RAS23 Wild type/tac-nifA

$+\quad 5725 \pm 150$

RAS24 Wild type/Ptac-nifL' nifA

$3400 \pm 200$

RAS24 Wild type/Ptac-nifL nifA +

$2800 \pm 150$ 


\begin{tabular}{|c|c|c|c|}
\hline RAS $15^{c}$ & fnr $r^{-}$Ptac-nifA & - & $5300 \pm 200$ \\
\hline RAS15 ${ }^{\mathrm{c}}$ & fnr $/$ Ptac-nifA & + & $5130 \pm 150$ \\
\hline $\operatorname{RAS} 25^{c}$ & fnr/Ptac-nifL- nifA & - & $3200 \pm 200$ \\
\hline RAS25 ${ }^{c}$ & fnr/Ptac-nifL- nifA & + & $3400 \pm 100$ \\
\hline RAS14 ${ }^{\mathrm{c}}$ & fnr/Ptac-nifLA & - & $160 \pm 10$ \\
\hline RAS14 ${ }^{\mathrm{c}}$ & fnr/Ptac-nifLA & + & $40 \pm 5$ \\
\hline RAS $16^{c}$ & fnr-Ptac-nifLA/Ptet-fnr & - & $3200 \pm 100$ \\
\hline $\operatorname{RAS} 17^{\circ}$ & $\begin{array}{l}\text { fnr/Ptac- } \\
\text { nifLA/pACYC184 }\end{array}$ & - & $190 \pm 10$ \\
\hline
\end{tabular}

a, data presented present mean values of three independent experiments

b, Strains contain the fnr null allele from M182 (fnr::Tn10) (Jayaramann et al., 1988)

', Strains contain the fnr null allele from RM101 (Sawers and Suppmann, 1992)

d, Determined by He et al. (1997)

nifL nifA, nifL ${ }^{\mathrm{C} 184 \mathrm{~S} / \mathrm{C} 187 \mathrm{~s}}$ nifA (see Materials \& Methods); Plac, Ptac or Ptet, under the control of the lac, tac or tet promoter, respectively.

In the case of NifA synthesis in the fnr strain in the absence of NifL (RAS8), however, the Bgalactosidase synthesis rate under anaerobic conditions was not significantly altered compared to the parental strain NCM1527 $\left(4800 \pm 100 \mathrm{U} / \mathrm{ml} \mathrm{OD}_{600}\right.$ and $5300 \pm 200 \mathrm{U} / \mathrm{ml}$ $\mathrm{OD}_{600}$, respectively) and was not affected by oxygen (Table 3). This indicates that the observed Fnr effect is mediated by NifL towards NifA in RAS7. However, nif expression under anaerobic conditions by NifA induced from the tac promoter in the absence of NifL synthesis using pJES851 (NCM1527) is significantly higher than using plasmid pNH3 (NCM1528), in which NifA expression depends on NifL synthesis based on translational coupling in the nifLA operon (Govantes et al., 1998). In addition western blot analysis showed that under our experimental conditions NifA amounts synthesized in NCM1527 were 
approximately 30 - $40 \%$ higher compared to NifA amounts synthesized in NCM1528 (data not shown). To rule out that nif expression in the fnr mutant using pJES851 (RAS8) is not due to this increase in NifA expression we additionally constructed pRS107 containing nifL ${ }^{\mathrm{C} 184 \mathrm{~S} / \mathrm{C187 \textrm {S }}}$ nifA translationally coupled under the control of the tac promoter (see Materials and Methods). IPTG induction in NCM1529 containing pRS107 (RAS2) resulted in NifA expression comparable to NCM1528 (data not shown) and expression of NifL ${ }^{\mathrm{C} 184 \mathrm{~S} / \mathrm{C} 187 \mathrm{~S}}$, which completely lost its nitrogen and oxygen regulatory function (Klopprogge and Schmitz, unpublished). Determination of B-galactosidase synthesis rates showed, that nif-induction by NifA expressed from pRS107 in the absence of a functional NifL protein was again not affected by the fnr mutation (compare RAS2 with RAS6) and was in the range of nif induction in NCM1528 under anaerobic conditions (Table 3). These findings indicate that the fnr null allele is not affecting NifA activity directly in the absence of functional NifL. In the presence of both regulatory proteins, however, NifL inhibits NifA activity under oxygenlimiting conditions when Fnr is absent, suggesting that the Fnr effect is mediated through NifL to NifA.

The finding that in the absence of Fnr NifL inhibits NifA activity under oxygen-limiting conditions to the same amount as under aerobic growth conditions indicates that NifL apparently does not receive the signal of anaerobiosis, when Fnr is absent. To confirm this observation, we analyzed the nif-induction under anaerobic conditions in a different $f n r$ mutant strain (RAS13). After introduction of nifLA, nifA and nifL $L^{\mathrm{C} 184 \mathrm{~S} / \mathrm{C} 187 \mathrm{~s}}$ nifA and on plasmids, the respective strains RAS14, RAS15 and RAS25 were grown under oxygenlimitation. By determining the $\beta$-galactosidase synthesis rates from the nifH'-promoter in RAS14, we observed that in this independent fnr mutant strain the nif-induction was $160 \pm 10$ $\mathrm{U} / \mathrm{ml} \mathrm{OD} \mathrm{OD}_{60}$, when nifLA was expressed under anaerobic conditions. This nif-induction is again significantly lower than in the parental strain RAS22 $\left(3500 \pm 80 \mathrm{U} / \mathrm{ml} \mathrm{OD}_{600}\right)$ and is in the range of aerobic nif-induction in the parental strain $\left(70 \pm 5 \mathrm{U} / \mathrm{ml} \mathrm{OD}_{600}\right)($ Table 3). Similar to RAS8 and RAS6 the B-galactosidase synthesis rate in the case of NifA synthesis in the absence of a functional NifL was not affected by the fnr mutation (RAS15 compared to RAS23 and RAS25 compared to RAS24). 
A $\quad$ B

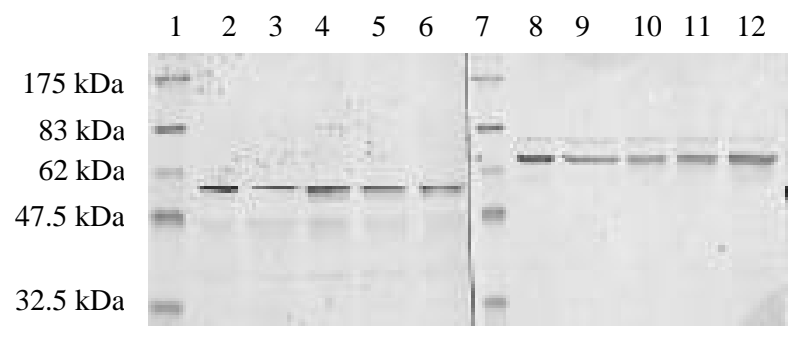

Figure 4: Amounts of NifA and NifL in wild type and $\boldsymbol{f n r} \boldsymbol{r}^{-}$strains of $\boldsymbol{E}$. coli. Cultures were grown at $30^{\circ} \mathrm{C}$ in minimal medium under anaerobic conditions with $4 \mathrm{mM}$ glutamine as limiting nitrogen source. The strains carried K. pneumoniae NifL and NifA under the control of the tac promoter on pNH3. Expression of NifL and NifA was induced with $10 \mu \mathrm{M}$ IPTG in wild type strain (lanes 2 and 8), in fnr null allele strains, RAS7 (lanes 3 and 9) and RAS14 (lanes 5 and 11), and in complemented strains RAS10 (lanes 4 and 10) and RAS16 (lanes 6 and 12). Amounts of NifL (A) and NifA (B) were determined by Western blotting. Prestained protein marker broad range (lanes 1 and 6) was purchased from New England Biolabs (UK).

The $f n r$ null alleles are not affecting the synthesis of NifL and NifA. To demonstrate that the failure of the $f n r$ mutant strains to express nifH under anaerobic conditions could not be accounted for by a decreased amount of NifA protein, we determined the amounts of NifA and NifL protein in the wild type and fnr mutant strains by immunological means. As shown in Figure 4 we observed no obvious differences in the amounts of the regulatory proteins of K. pneumoniae in the different $f n r^{-}$backgrounds compared to the parental strains.

Fnr is required for release of NifL inhibition of NifA activity under anaerobic conditions in the heterologous $\boldsymbol{E}$. coli system. To determine if constitutive expression of $f n r$ is able to restore nif-induction in the $f n r$ mutant strains we expressed E. coli fnr from the tet promoter (pRS120) or the lac promoter (pRS79) in addition to the nifLA operon. Expression of Fnr in trans from either promoter resulted in complementation with a restoration of anaerobic growth on nitrate and glycerol (data not shown). It further resulted in relief of NifL inhibition of NifA activity under oxygen-limiting conditions. This restoration of nif-induction 
was achieved in both strains carrying independent chromosomal $f n r$ null alleles (RAS10 and RAS16, respectively) which is displayed graphically in Figure 5. The nif induction under anaerobic conditions in both mutant strains was restored to the induction level of the parental strains (NCM1528 and RAS22, respectively) by expressing E. coli fur from promoter Ptet on pACYC184 or promoter Plac on pMCL210, whereas the vectors pACYC184 and pMCL210 alone did not restore nif-induction (Table 3). These results and the finding that Fnr affects NifA only in the presence of NifL (see above) strongly indicate that in the heterologous E. coli system, Fnr is required for release of NifL inhibition of NifA activity under anaerobic conditions.
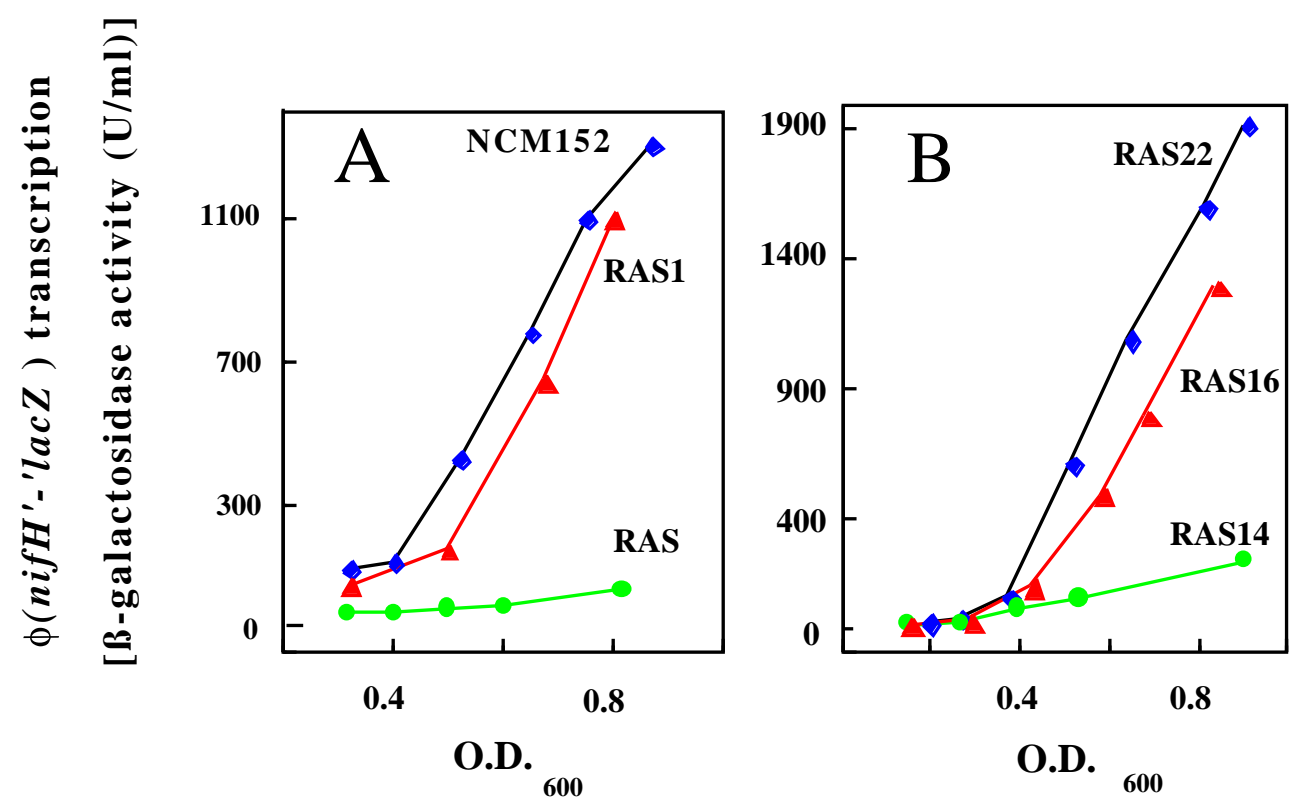

Figure 5: Effects of $f n r$ null alleles on expression of a $\emptyset\left(n i f H^{\prime}\right.$-'lacZ) fusion in heterologous $E$. coli strains carrying K. pneumonia nifLA on a plasmid. The activity of $\beta$-galactosidase was plotted as a function of OD600 for cultures grown at $30^{\circ} \mathrm{C}$ in minimal medium under anaerobic conditions with $4 \mathrm{mM}$ glutamine as limiting nitrogen source. Differential rates of transcription from the nifH promoter, which reflect NifA activity, were determined from the slopes of these plots. All strains carried a single copy of a $\varnothing$ (nifH' -'lacZ) fusion at the trp locus (He et al. 1997) and plasmid pNH3 encoding NifL and NifA under the control of the tac promoter. A fnr null allele transduced from M182 ( $f n r:: T n 10)$ : Wild type NCM1528 (diamonds), the respective fnr null allele in NCM1528 (RAS7) (circles), complemented respective fnr mutant by constitutive expression of E. coli fnr on pACYC184 (RAS10) (triangles). B fnr null allele from RM101: Wild type RAS22 (diamonds), the respective fnr 
null allele in RAS22 (RAS14) (circles), complemented respective fnr mutant by constitutive expression of E. coli fnr on pACYC184 (RAS16) (triangles).

The wild type strain (NCM1528) grown in the presence of $10 \mathrm{mM}$ ammonium showed nif inductions of approximately $3 \pm 1 \mathrm{U} / \mathrm{ml} \mathrm{OD}_{600}$ independent of the oxygen availability (data not shown). This induction level is significantly lower than the nif-induction observed in the fnr mutant strains (RAS7 and RAS14) under oxygen- and nitrogen-limiting growth conditions ( $100 \pm 10 \mathrm{U} / \mathrm{ml} \mathrm{OD}_{600}$ and $160 \pm 10 \mathrm{U} / \mathrm{ml} \mathrm{OD}_{600}$, respectively). These data suggest that Fnr is required for the oxygen signal transduction to NifL rather than for the ammonium signal transduction. They further indicate that in the absence of Fnr NifL apparently does not receive the signal for absence of oxygen and therefore inhibits NifA activity under anaerobic conditions.

\section{$\Omega$}

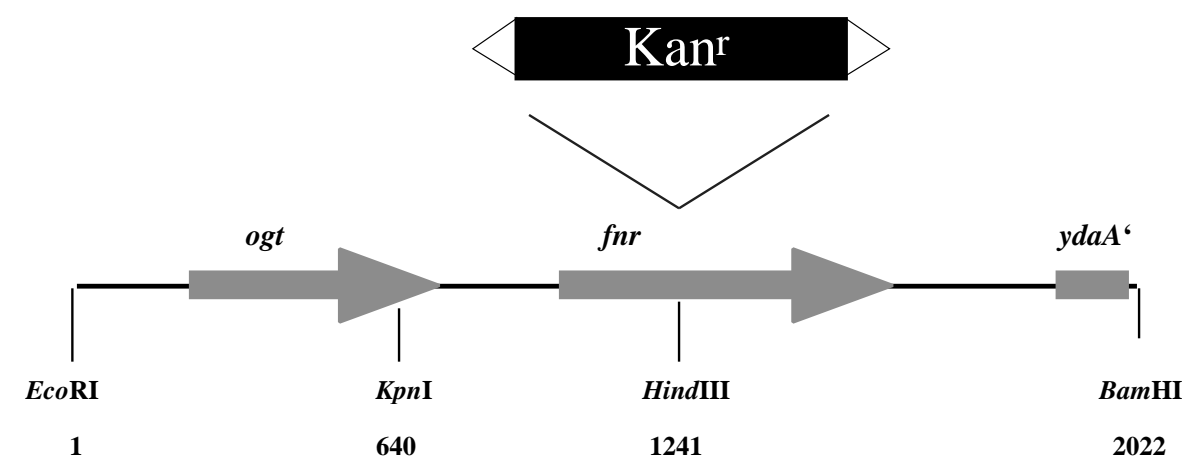

Figure 6: Map of the cloned EcoRI-BamHI fragment (pRS127) showing the side of insertion of the $\Omega$ interposon

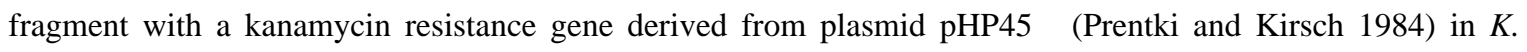
pneumoniae fnr. The $\Omega$ interposon fragment is flanked by short inverted repeats including strong transcription termination signals. The sequence of the EcoRI-BamHI fragment has been submitted to GenBank under accession number AF220669.

Studying the effect of Fnr on the nif-induction in K. pneumoniae. In order to confirm the requirement of Fnr for relief of NifL inhibition under anaerobic conditions in the heterologous 
E. coli system, we constructed a chromosomal fnr null allele in $K$. pneumoniae. We used K. pneumoniae strain UN4495 carrying nifLA and a nifK-lacZ fusion on the chromosome, which allows monitoring of NifA-mediated transcription from the nifHDK-promoter by measuring the differential rate of B-galactosidase synthesis (Schmitz et al., 1996). The fnr deletion was constructed on a plasmid by inserting an $\Omega$ interposon fragment with a kanamycin resistance gene into K. pneumoniae fnr (Figure 6), which was than introduced into the chromosome by marker exchange using the sac system (see Materials and Methods). The disruption of the fnr gene was confirmed by PCR and southern blot analysis (data not shown). Klebsiella strains with the exception of RAS26 and RAS27 were generally grown in minimal medium under nitrogen limitation to exclude NifA inhibition by NifL in response to ammonium. The $f n r:: \Omega$ mutation in $K$. pneumoniae UN4495 did not result in a significant growth-rate reduction, but did reduce the nif-induction under oxygen-limiting conditions to 10 $\%$ of the nif-induction in the parental strain. The observed induction level of the K. pneumoniae fnr mutant strain (RAS18) under anaerobic conditions $\left(400 \pm 20 \mathrm{U} / \mathrm{ml} \mathrm{OD}_{600}\right)$ again is in the same range as the nif-induction in the presence of oxygen in the parental $K$. pneumoniae strain $\left(220 \pm 20 \mathrm{U} / \mathrm{ml} \mathrm{OD}{ }_{600}\right)$ (Table 4). Determination of NifA and NifL proteins in the fnr mutant strain revealed no differences in the amount of the regulatory proteins compared to the parental strain (data not shown), indicating that the failure to express nifH could not be accounted for by a decrease of NifA expression. Normal NifL/NifAdependent regulation was restored by introduction of the K. pneumoniae fnr gene expressed from the tet promoter on pRS137 into the fnr mutant (Figure 7). nif induction in the complemented mutant (RAS19) was determined to be $3800 \pm 50 \mathrm{U} / \mathrm{ml} \mathrm{OD}_{600}$, whereas the low copy vector pACYC184 alone did not result in complementation (RAS20). These findings in the native background again suggest that Fnr is required for nif expression in K. pneumoniae under anaerobic conditions. 
Table 4: Effects of a fnr:: $\Omega$ mutation on NifL activity in K. pneumoniae UN4495.

\begin{tabular}{|c|c|c|c|c|c|}
\hline Strain & Relevant genotype & $\begin{array}{l}\text { Nitrogen } \\
\text { source }\end{array}$ & $\begin{array}{c}\text { Presence } \\
\text { of } \\
\text { oxygen }\end{array}$ & $\begin{array}{c}\text { Expression of } \\
\text { nifH'-'lacZ' }^{\prime} \\
\left(\mathrm{U} / \mathrm{ml} \cdot \mathrm{O.D}_{{ }_{600}}\right)^{\mathrm{a}}\end{array}$ & $\begin{array}{c}\text { Doubling } \\
\text { time } \\
\text { (h) }\end{array}$ \\
\hline UN 4495 & Wild type & glutamine & - & $4400 \pm 100$ & 3.5 \\
\hline UN 4495 & Wild type & glutamine & + & $220 \pm 10$ & 2.0 \\
\hline RAS18 & $f n r^{-}$ & glutamine & - & $400 \pm 20$ & 4.0 \\
\hline RAS18 & $f n r^{-}$ & glutamine & + & $100 \pm 10$ & 2.2 \\
\hline RAS19 & fnr / Ptet-fnr $r^{\mathrm{b}}$ & glutamine & - & $3800 \pm 50$ & 3.8 \\
\hline RAS20 & fnr / pACYC184 & glutamine & - & $660 \pm 30$ & 4.2 \\
\hline RAS26 & $\begin{array}{l}\text { Wild type / Ptac-nifL } \\
\text { nifA }\end{array}$ & ammonium $^{c}$ & - & $2350 \pm 100$ & 3.7 \\
\hline RAS26 & $\begin{array}{l}\text { Wild type / Ptac-nifL } \\
\text { nifA }\end{array}$ & ammonium $^{c}$ & + & $2100 \pm 100$ & 1.7 \\
\hline RAS27 & fnr / Ptac-nifL nifA & ammonium $^{\mathrm{c}}$ & - & $2200 \pm 50$ & 4.1 \\
\hline RAS27 & fnr / Ptac-nifL- nifA & ammonium $^{\mathrm{c}}$ & + & $2150 \pm 150$ & 1.6 \\
\hline RAS28 & Wild type / Ptac-nifLA & glutamine & - & $2400 \pm 30$ & 4.0 \\
\hline RAS28 & Wild type / Ptac-nifLA & glutamine & + & $160 \pm 5$ & 1.6 \\
\hline RAS29 & fnr / Ptac-nifLA & glutamine & - & $430 \pm 30$ & 3.6 \\
\hline RAS29 & fnr / Ptac-nifLA & glutamine & + & $310 \pm 30$ & 1.6 \\
\hline RAS30 & $\begin{array}{l}\text { 4495 } \text { nifLA / Ptac- } \\
\text { nifLA }\end{array}$ & glutamine & - & $2450 \pm 30$ & 4.1 \\
\hline
\end{tabular}

a, data presented represent mean values of three independent experiments

b, K. pneumoniae fnr is expressed under the control of the tet promoter (Ptet)

${ }^{c}$, grown in the presence of $10 \mathrm{mM}$ ammonium to repress chromosomal nifLA induction

nifL nifA, nifL ${ }^{\mathrm{C} 184 \mathrm{~S} / \mathrm{C} 187 \mathrm{~S}}$ nifA (see Materials \& Methods); Ptac, under the control of the tac promoter. 
In order to confirm our finding observed in the heterologous E. coli system, that Fnr is required to relieve NifL inhibition of NifA activity under anaerobic conditions, we studied the effect of the fnr null allele on NifA in Klebsiella. Plasmid pRS159 carrying nifL $L^{\mathrm{C} 184 \mathrm{~S} / \mathrm{C} 187 \mathrm{~s}}$ nifA translationally coupled under the control of the tac promoter was introduced into K. pneumoniae UN4495 and the corresponding fnr mutant strain RAS18. As growth in minimal medium in the presence of $10 \mathrm{mM}$ ammonium results in repression of the chromosomal nifLA operon, under nitrogen sufficieny only nif $L^{\mathrm{C} 184 \mathrm{~S} / \mathrm{C} 187 \mathrm{~s}}$ nifA from pRS159 is induced, resulting in the synthesis of NifA and a non-functional NifL protein (see above). Determination of B-galactosidase synthesis rates under those conditions in the fnr mutant strain (RAS27) and the parental strain (RAS26) showed that the absence of Fnr under anaerobic conditions is not affecting NifA activity in the absence of a functional NifL protein $\left(2200 \pm 50 \mathrm{U} / \mathrm{ml} \mathrm{OD}_{600}\right.$ and $2350 \pm 100 \mathrm{U} / \mathrm{ml} \mathrm{OD}_{600}$, respectively) (Table 4). These results indicate that the Fnr effect on nif regulation observed in the native background is based on the Fnr requirement for relief of NifL inhibition under oxygen-limiting growth conditions. Based on our findings, we hypothesize that in K. pneumoniae, Fnr is the primary oxygen sensor for the nif regulation, which transduces the signal directly or indirectly to NifL.

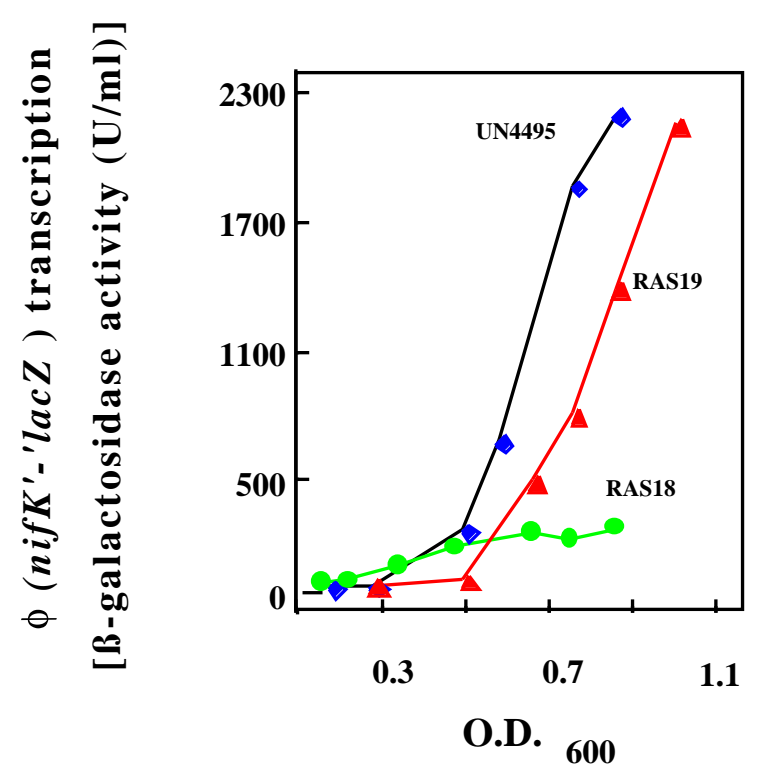

Figure 7: Effects of an fnr null allele on expression of a nifK-LacZ fusion in K. pneumoniae strain UN4495.

The activity of $\beta$-galactosidase was plotted as a function of $\mathrm{OD}_{600}$ for cultures grown at $30^{\circ} \mathrm{C}$ in minimal medium under anaerobic conditions with $4 \mathrm{mM}$ glutamine as limiting nitrogen source. Differential rates of 
transcription from the nifHDK promoter were determined from the slopes of these plots. Wild type UN4495 (diamonds), the fnr mutant strain of UN4495 (RAS18) (circles), complemented respective fnr mutant by constitutive expression of $K$. pneumoniae fnr on pACYC184 (RAS19) (triangles).

\section{Discussion}

Our goal is to determine how K. pneumoniae NifL perceives the oxygen status of the cells in order to regulate NifA activity in response to environmental oxygen. The main question concerning the oxygen signal transduction is whether NifL senses oxygen directly via a redox-induced conformational change, or whether oxygen is detected by a more general system. After receiving the oxygen signal, directly or indirectly, the redox state of the flavoprotein NifL is thought to influence the ability of NifL to modulate the NifA activity in response to environmental oxygen, and to allow NifA activity only in the absence of oxygen (Hill et al., 1996; Macheroux et al., 1998; Schmitz 1996). We recently showed that iron is specifically required for nif-induction, but is not present in NifL (Schmitz, 1997; Schmitz et al., 1996). To determine whether this iron requirement for nif induction could be accounted for by the role of Fnr in transducing the oxygen signal to NifL, we determined the effect of an fnr null allele on nif regulation. Using different genetic backgrounds and independent fnr null alleles, we were able to show that the absence of Fnr effects the nif regulation dramatically. The nif-induction in the absence of Fnr was low, similar to the nif-induction under aerobic conditions, even though cells were growing under oxygen limitation. Normal nif regulation was achieved in the mutant strains by introduction of a low-copy vector expressing $f n r$ constitutively (Figures 6 and 8). These data indicate that Fnr is required to relieve NifL inhibition of NifA activity under anaerobic conditions and this appears to account for the iron requirement of nif induction (Schmitz et al.,1996). Therefore, in addition to the rhizobial homologous Fnr proteins, FnrN and FixK, which are known to be involved in regulation of nitrogen fixation in the symbiotic bacteria (Fischer, 1994 and therein cited papers, Guiterrez et al., 1997), in K. pneumoniae the transcriptional activator Fnr is apparently also involved in regulation of nitrogen fixation. These results are in contrast to the report of Hill (1985), that 
redox regulation of nif expression in a heterologous E. coli strain is independent of the E. coli fur gene product. This discrepancy may be due to experimental differences. We determined NifA-mediated transcriptional activation by measuring differential rates of $\beta$-galactosidase expression from a chromosomal nifK-lac $Z$ fusion in order to monitor nif induction. In contrast, Hill determined acetylene reduction by nitrogenase after growing heterologous E. coli fnr strains carrying the Nif+ plasmid pRD1 under derepressing conditions. Also, as plasmid pRD1 contains in addition to the nif genes non-identified $K$. pneumoniae genes (Dixon et al., 1976) we cannot completely rule out that $K$. pneumoniae fnr is encoded on the plasmid. Apart from these experimental differences concerning the heterologous E. coli systems we confirmed the Fnr requirement for the nif regulation in the native genetic background K. pneumoniae.

We further showed that the general oxygen sensor Fnr is required for relief of NifL inhibition under anaerobic growth conditions and that the presence of ammonium results in significantly lower nif-inductions in the wilde type strain than observed in $f n r$ mutant strains under nitrogen- and oxygen-limitation. Both these findings suggest, that the oxygen signal is not detected by NifL directly but by Fnr, which transduces the signal - directly or indirectly - to NifL. However, at this state of experimental data we cannot completely rule out that the Fnr requirement might be due to some Fnr-dependent metabolic signals not directly related to the lack of oxygen. If Fnr is indeed the primary oxygen sensor for the nif regulation in K. pneumoniae, it still remains to be explained how the oxygen signal is transmitted to NifL. Fnr is either transducing the oxygen signal by directly interacting with NifL in the absence of oxygen or under anaerobic conditions Fnr is activating the transcription of gene(s) whose product(s) mediate the signal to NifL. As Fnr is a transcriptional activator and can be excluded as the physiological electron donor for NifL reduction it is more reasonably that under anaerobic conditions Fnr transduces the signal by transcriptional activation. 


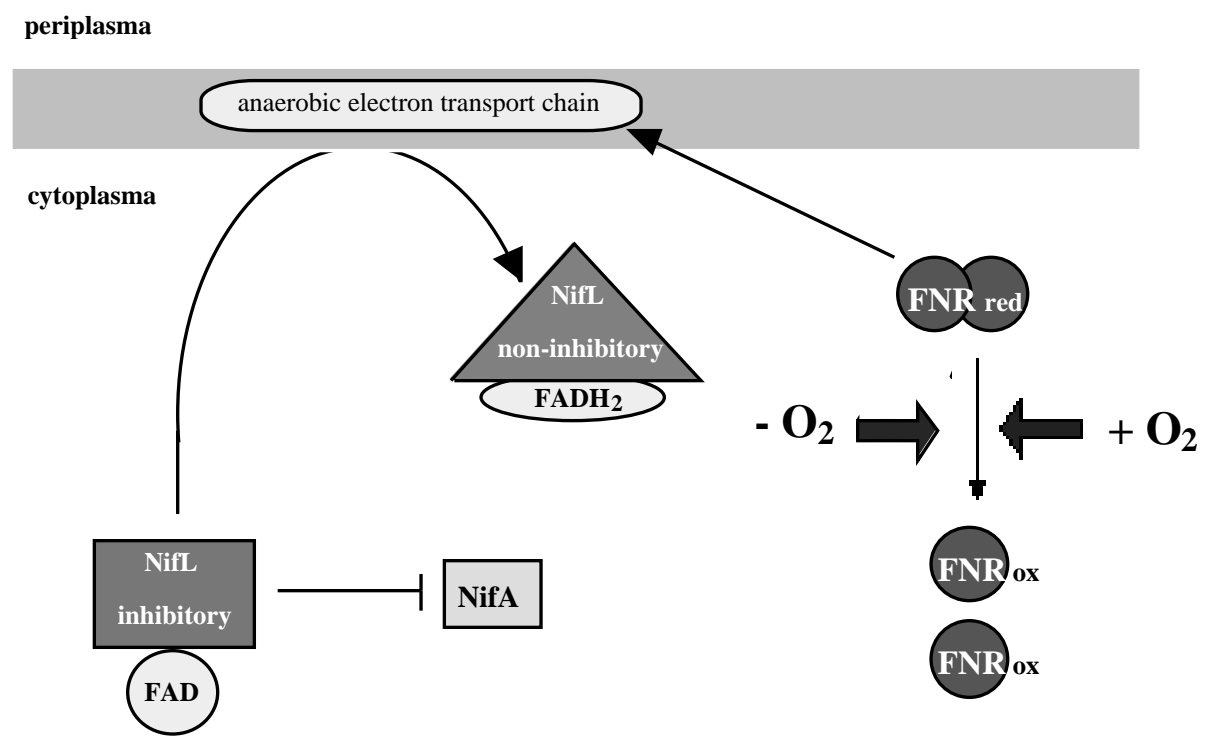

Figure 8: Hypothetical model of oxygen signal transduction in K. pneumoniae.

Hypothetical model for oxygen signal transduction. In K. pneumoniae, as in A. vinelandii, the redox state of the flavoprotein NifL is thought to influence its ability to modulate the NifA activity in response to the oxygen levels. However, the physiological electron donors for NifL have not yet been identified (Klopprogge and Schmitz, 1999, Macheroux et al., 1998). If the redox state of the flavoproteins is indeed responsible for mediating the oxygen signal to NifA, one could postulate that by reducing the cofactor of NifL, the physiological electron donor is transducing the oxygen signal to NifL. Thus, the physiological electron donor for the NifL reduction may be a component of the oxygen signal transduction. As one can exclude Fnr as the physiological electron donor for NifL reduction in the absence of oxygen, one has to postulate another downstream signal transductant following Fnr. We therefore hypothesize that in the absence of oxygen, Fnr activates transcription of gene(s) whose product(s) function to relieve NifL inhibition by reducing the FAD cofactor of NifL. Attractive hypothetical candidates for the physiological electron donor for NifL are components of the anaerobic electron transport system (Fig. 8), particularly the electron transport system to fumarate, whose transcription under anaerobic conditions is directly dependent on Fnr activation (Ackrell, 2000; Manodori et al., 1992; Skotnicki and Rolfe, 1979; Van Hellemond and Tielens, 1994). 
Preliminary data, which indicate that $K$. pneumoniae NifL under anaerobic conditions is membrane-associated, whereas in the presence of oxygen NifL is in the cytosolic fraction, support this model (Klopprogge and Schmitz, unpublished). Studies of the anaerobic electron transport system components as potential physiological electron donors for NifL are in process. 


\title{
Chapter 4:
}

\section{Membrane association of Klebsiella pneumoniae $\mathrm{NifL}$ is affected by molecular oxygen and combined nitrogen}

\author{
KAI KLOPPROGGE, ROMAN GRABBE, MICHEAL HOPPERT, AND RUTH A. \\ SCHMITZ,
}

\begin{abstract}
In the diazotroph Klebsiella pneumoniae, NifL and NifA regulate transcription of the nitrogen fixation genes in response to molecular oxygen and combined nitrogen. We recently showed that Fnr is the primary oxygen sensor, which transduces the oxygen signal towards the negative regulator NifL by activating genes whose products reduce the FAD moiety of NifL under anaerobic conditions. Potentially, these Fnr-dependent gene products could be membrane-bound components of the anaerobic electron transport chain; consequently, in this study we now examine the localization of NifL within the cell under various growth conditions. In $K$. pneumoniae grown under oxygen- and nitrogen-limited conditions, approximately $55 \%$ of the total NifL protein were found in the membrane fraction. However, when the cells were grown aerobically or shifted to nitrogen sufficiency, less than $10 \%$ of total NifL was membrane-associated. In contrast to NifL, NifA was located in the cytoplasm under all growth conditions tested. Further studies using $K$. pneumoniae mutant strains showed that under derepressing conditions but in the absence of either the primary oxygen sensor Fnr or the primary nitrogen sensor GlnK and the ammonium transporter AmtB, NifL was located in the cytoplasm and inhibited NifA activity. These findings suggest that under nitrogen- and oxygen-limitation, a significant higher membrane affinity of NifL may create a spatial gap between NifL and its cytoplasmic target protein NifA thereby impairing inhibition of NifA by NifL. Localization of GlnK further showed that under nitrogen-limited conditions, but independent of oxygen presence, $15-20 \%$ of the total GlnK is membrane associated.
\end{abstract}

\section{Introduction}

The free-living diazotroph Klebsiella pneumoniae is able to fix molecular nitrogen under anaerobic and nitrogen-limited growth conditions. To avoid unnecessary consumption of energy synthesis of nitrogenase is tightly controlled by the regulatory nitrogen fixation operon nifLA (for review see Dixon, 1998; Schmitz et al., 2001). Products of the nifLA operon regulate transcription of the other nif genes in response to environmental signals. NifA is the 
transcriptional activator of all of the nif operons except the nifLA operon, which is under control of the global nitrogen regulatory system, ntr (Drummond and Wootton, 1987; Blanco et al., 1993); NifL antagonizes the transcriptional activity of NifA in response to combined nitrogen and molecular oxygen by direct protein-protein interaction with NifA (Merrick et al., 1982; Hill et al., 1981; Money et al., 1999; Lei et al., 1999; Money et al., 2001; Little et al., 2000, Barrett et al., 2001).

External nitrogen availability is apparently perceived by $K$. pneumoniae through changes in the internal glutamine pool (Schmitz, 2000), which are subsequently mediated towards the nif regulatory system. Recent evidence strongly suggests that the nitrogen status of the cells is transduced towards the NifL/NifA regulatory system in K. pneumoniae and Azotobacter vinelandii by the GlnK protein, a paralogue PII-protein (He et al., 1998; Jack et al., 1999; Arcondéguy et al., 1999 and 2000; Little et al., 2000; Reyes-Ramirez et al., 2001). The effect of GlnK, which apparently interacts with NifL or affects the NifL/NifA-complex via direct protein-protein interaction, appears to be contradictory in $K$. pneumoniae and $A$. vinelandii (He et al., 1998; Jack et al., 1999; Little et al., 2000; Reyes-Ramirez et al., 2001). The oxygen signal is received by NifL, which contains an $N$-terminally bound flavin adenine dinucleotide (FAD) as a prosthetic group. Recent work has shown that the flavoprotein NifL acts as a redox-sensitive regulatory protein, which modulates NifA activity in response to the redox state of its FAD cofactor and allows NifA activity only in the absence of oxygen (Hill et al., 1996; Schmitz, 1997; Macheroux et al., 1998; Little et al., 2000). Thus, under anaerobic conditions in the absence of combined nitrogen, reduction of the flavin moiety of NifL results in a non-inhibitory conformation of the NifL protein. We have recently shown that in $K$. pneumoniae, the global transcriptional regulator Fnr is required to mediate the signal of anaerobiosis to NifL (Grabbe et al., 2001b). Thus, we proposed that in the absence of oxygen the primary oxygen sensor Fnr activates transcription of a gene or genes whose product or products reduce the FAD cofactor of NifL, resulting in a non-inhibitory conformation of the protein, assuming the absence of a sufficient nitrogen source. Candidates for the physiological electron donor for NifL reduction include those components of the anaerobic electron transport system with Fnr-dependent synthesis (Grabbe et al., 2001b). This model implies that under anaerobic conditions, NifL will contact the cytoplasmic membrane during the reduction of its flavin cofactor. If NifL reduction indeed occurs by a membrane-associated electron donor, this provides a potential mechanism for the signal transduction of anaerobiosis that is similar to the signal transduction of oxygen presence proposed for the Escherichia coli FADcontaining aerotaxis protein Aer (Bibikov et al. 1997; Rebbapragada et al., 
1997).

Our goal is to analyze the reduction process of NifL-bound FAD and the subsequent conformational change of the protein. We therefore localized of NifL protein in $K$. pneumoniae and $K$. pneumoniae mutant strains grown under various conditions. We report here that under derepressing conditions, NifL shows a significantly higher association with the cytoplasmic membrane than in the presence of either molecular oxygen or combined nitrogen. As the presence of molecular oxygen or combined nitrogen results in nif gene repression, these findings imply that a spatial separation of cytoplasmic NifA and its antagonist NifL may be responsible for nif gene induction under oxygen- and nitrogen-limited conditions.

\section{Materials and methods}

\section{Bacterial strains and plasmids}

K. pneumoniae strains M5al (wild-type), M5al containing plasmid pNH3, and K. pneumoniae UN4495 (ø(nifK-lacZ)5935 $\Delta$ lac-4001 hisD4226 Galr) (MacNeil et al., 1981) and mutant derivatives (K. pneumoniae UN4495 fnr:: $\Omega$ (RAS18, Grabbe et al., 2001b), K. pneumoniae UN4495 $g \ln K:$ KIXX) were used in this study. The $g \ln K:$ KIXX allele was transferred from K. pneumoniae UNF3433 (Jack et al., 1999) into K. pneumoniae UN4495 by P1-mediated transduction with selection for kanamycin resistance, resulting in RAS36. Plasmid pNH3 carries the K. pneumoniae nifLA operon under the control of the tac promoter (Henderson et al., 1989).

\section{Growth conditions}

$K$. pneumoniae strains were grown anaerobically with molecular nitrogen $\left(\mathrm{N}_{2}\right)$ as gas phase at $30^{\circ} \mathrm{C}$ in minimal medium supplemented with either $2 \mathrm{mM}$ glutamine (nitrogen limitation) or $10 \mathrm{mM}$ ammonium (nitrogen sufficiency) as the sole nitrogen source, $0.004 \%$ histidine, 10 $\mathrm{mM} \mathrm{Na} \mathrm{CO}_{3}, 0.3 \mathrm{mM}$ sulfide and $1 \%$ sucrose as the sole carbon source (Schmitz et al., 1996). To monitor anaerobiosis, the medium was further supplemented with $0.002 \%$ resazurin. Precultures were grown overnight in closed bottles with $\mathrm{N}_{2}$ as gas phase in the same medium but lacking sulfide and resazurin. The 1-1 main cultures were inoculated from precultures and incubated in closed bottles with molecular nitrogen as gas phase under strictly anaerobic conditions without shaking. Samples were taken anaerobically for monitoring the optical density at $600 \mathrm{~nm}$ and determining B-galactosidase activity. Aerobic 1-1 cultures were incubated in 2-1 flasks with vigorous shaking $(130 \mathrm{rpm})$ using the same medium and culture 
supplements as described for the anaerobic growth but lacking $\mathrm{Na}_{2} \mathrm{CO}_{3}$, sulfide and resazurin. For ammonium shift experiments, 1-1 cultures growing under nitrogen limitation in the presence of $2 \mathrm{mM}$ glutamine were shifted to nitrogen sufficiency by the addition of $10 \mathrm{mM}$ $\mathrm{NH}_{4} \mathrm{Cl}$ during mid-exponential growth; the shifted cultures were further incubated for $2 \mathrm{~h}$ before the cells were harvested.

\section{$\beta$-Galactosidase assay}

NifA-mediated activation of transcription from the nifHDK promoter in $K$. pneumoniae UN4495 and mutant derivatives (UN4495 $f n r:: \Omega$ and UN4495 $g \ln K:: \mathrm{KIXX}$ ) was monitored by measuring the differential rate of $\beta$-galactosidase synthesis during exponential growth $(\mathrm{U}$ $\left.\mathrm{ml}^{-1}\left(\mathrm{OD}_{600}\right)^{-1}\right)$ as described by Schmitz et al. (1996). Inhibitory effects of NifL on NifA activity were assessed by a decrease in nifH expression.

\section{Electron microscopy}

For electron microscopy, K. pneumoniae strain M5al carrying a plasmid-borne nifLA-operon under the control of the tac promoter was used (pNH3, Henderson et al., 1989) in addition to the chromosomal nifLA operon. Cultures $(50 \mathrm{ml})$ were incubated anaerobically in minimal medium supplemented with either $2 \mathrm{mM}$ glutamine or $10 \mathrm{mM} \mathrm{NH}_{4} \mathrm{Cl}$ as the sole nitrogen source, as described above. During growth additional NifL and NifA synthesis from the plasmid was induced by the addition of $10 \mu \mathrm{M}$ IPTG. When cultures reached an $\mathrm{OD}_{600}$ of 0.8 , cells were harvested by centrifugation at $10.000 \mathrm{x} \mathrm{g}$ under anaerobic conditions. The resulting cell pellet was resuspended in $50 \mathrm{mM}$ potassium phosphate buffer, $\mathrm{pH} 7.3$, and cells were chemically fixed in $0.2 \%(\mathrm{w} / \mathrm{v})$ formaldehyde and $0.3 \%(\mathrm{w} / \mathrm{v})$ glutardialdehyde solution for $90 \mathrm{~min}$ at $0{ }^{\circ} \mathrm{C}$ in a closed reaction cup under anaerobic conditions. Finally, the cells were dehydrated in a graded methanol series and embedded in Lowicryl K4M resin under air (Roth et al., 1981; Hoppert and Holzenburg, 1998). Resin sections of 80 - $100 \mathrm{~nm}$ in thickness were cut with glass knives. NifL was localized in resin sections using specific polyclonal antisera directed against NifL and goat-anti-rabbit-IgG linked to colloidal gold (10 $\mathrm{nm}$ in diameter, BBI, Cardiff, UK), essentially as described by Roth et al. (1978) with some modifications (Hoppert and Holzenburg, 1998). Electron micrographs were taken, at calibrated magnifications, with a Philips EM 301 (Philips, Eindhoven, The Netherlands).

\section{Membrane preparation}


To localize of NifL synthesized from the single chromosomal copy of the nifL gene, cytoplasmic and membrane fractions of $K$. pneumoniae UN4495 and mutant derivatives were separated by several centrifugation steps. Membranes from anaerobically grown cells were prepared under strictly anaerobic conditions in the presence of $2 \mathrm{mM}$ dithiothreitol under a nitrogen atmosphere; aerobic membranes were prepared under aerobic conditions in the absence of dithiothreitol. To separate the membrane and cytoplasmic fractions, exponentially growing 1-1 cultures were harvested by centrifugation, resuspended in $30 \mathrm{ml} \mathrm{B}$ buffer $(2 \mathrm{mM}$ Epps ( $N$-[2-hydroxyethyl]piperazine- $N$ '-3-propanesulfonic acid), $25 \mathrm{mM}$ potassium glutamate, $5 \%$ glycerol, $\mathrm{pH} 8.0$ ) and disrupted using a French pressure cell. Cell debris were sedimented by centrifugation twice at $20,000 \mathrm{x}$ g for $30 \mathrm{~min}$ each time. The resulting cell-free cell extract was centrifuged twice at $120,000 \mathrm{x} \mathrm{g}$ for $2 \mathrm{~h}$ to sediment the membrane fraction. The membrane fraction was subsequently washed two times with $10 \mathrm{ml}$ B-buffer followed by centrifugation at $120,000 \mathrm{x} \mathrm{g}$ for $2 \mathrm{~h}$. The resulting supernatants of all ultracentrifugation steps were combined (a totat volume $50 \mathrm{ml}$ ), designated the cytoplasmic fraction and stored at $4{ }^{\circ} \mathrm{C}$ for further studies. The resulting hydrophobic pellets were resuspended in $10 \mathrm{ml} \mathrm{B}-$ buffer containing $3 \mathrm{mM}$ Triton X-100. The membrane-bound and membrane-associated proteins were solubilized out of the membrane fraction by incubating the resuspended membrane pellet for $30 \mathrm{~min}$ at $4{ }^{\circ} \mathrm{C}$ under vigorous shaking. After this solubilization step, the phospholipids were subsequently separated from the solubilized protein by centrifugation at $120,000 \mathrm{x} \mathrm{g}$ for $2 \mathrm{~h}$. The supernatant of a total volume of $10 \mathrm{ml}$ containing the solubilized proteins was designated the membrane fraction and stored at $4{ }^{\circ} \mathrm{C}$ for further studies. Protein concentration of the membrane and cytoplasmic fraction was determined via the method of Bradford (1976) with the BioRad protein assay using bovine serum albumin as standard.

The quality of the membrane preparations was evaluated by determination of the malate dehydrogenase activity in both the membrane and the cytoplasmic fraction, according to Bergmayer (1983). The oxidation of NADH was measured at room temperature in 1-ml test assays containing $100 \mathrm{mM}$ HEPES pH 7.4, $0.44 \mathrm{mM} \mathrm{NADH}$, and $100 \mu \mathrm{l}$ of the respective samples. The reactions were started by the addition of $1.8 \mathrm{mM}$ oxaloacetate. The oxidation of the NADH was monitored at $365 \mathrm{~nm}$ using a Jasco V550 UV/Vis-spectrophotometer. In addition, quinoproteins were specifically detected by a redox-cycle stain assay to detect leakage of membrane proteins into the cytoplasmic fraction. Aliquots $(5 \mu \mathrm{l})$ of membrane and cytoplasmic fractions were spotted on a nitrocellulose membrane and stained using $0.24 \mathrm{mM}$ nitroblue tetrazolium in $2 \mathrm{M}$ potassium glycinate $(\mathrm{pH} 10)$ as described by Flückiger et al. (1995). The nitrocellulose membrane was immersed in the nitroblue tetrazolium/glycinate 
solution in the dark for $45 \mathrm{~min}$, resulting in a blue-purple stain of quinoproteins. Subsequently protein was stained red with Ponceau $\mathrm{S}(0.1 \%$ in $5 \%$ acetic acid); the already-stained quinoproteins remained blue-purple.

\section{Western blot analysis and quantification of NifL, NifA and GInK in membrane and cytoplasmic fractions}

Samples of the membrane and cytoplasmic fractions were diluted 1:1 with gel-loading buffer containing or lacking SDS and subsequently separated by SDS-polyacrylamide (12\%) gel electrophoresis (Laemmli, 1970) or native polyacrylamide (12.5\%) gel electrophoresis (Atkinson et al., 1994), respectively. Prestained protein markers (New England Biolabs, UK) were used as molecular mass standards. After separation, proteins were transferred to nitrocellulose membranes as described previously (Sambrook et al., 1989). Membranes were exposed to specific polyclonal rabbit antisera directed against the NifL, NifA or GlnK proteins of $K$. pneumoniae. Polyclonal antibodies directed against NifL, NifA and GlnK from $K$. pneumoniae were specific for the $K$. pneumoniae proteins NifL, NifA and GlnK, respectively. Polyclonal GlnK antibody was used in a very high dilution range, conditions under which cross-reaction with GlnB was approximately negligible as confirmed by separating purified GlnB and GlnK by isoeletric focusing and western blot analysis.

Protein bands were detected with secondary antibodies directed against rabbit immunoglobulin $\mathrm{G}$ and coupled to horseradish peroxidase (BioRad Laboratories). The bands were visualized using the ECLplus system (Amersham Pharmacia) with a fluoroimager (Storm, Molecular Dynamics). The protein bands were quantified for each growth condition in three independent membrane preparations using the ImageQuant v1.2 software (Molecular Dynamics) and known amounts of the respective purified proteins. The calibration with purified K. pneumoniae proteins showed that quantification of NifL and NifA was linear within absolute amounts of 0.5 to $10 \mu \mathrm{g}$ per lane and GlnK within 0.5 to $5 \mu \mathrm{g}$; all quantifications of those proteins in K. pneumoniae cell fractions have been performed within this linear range of the detection system. For calculation of the protein amounts in the fractions the quantifications were normalized to the actual volume for both membrane and cytoplasmic fractions. This was done either by initially applying $20 \mu 1$ of the cytoplasmic and $4 \mu 1$ of the membrane fraction onto the gels or applying equal amounts of the fractions onto the gel and considering the higher total volume of the cytoplasmic fraction in the calculation. Relative amounts of protein in the respective fraction to total amount were calculated by setting the absolute amounts in both the cytoplasmic and membrane fraction of a membrane preparation as $100 \%$. 


\section{Analysis of GInK uridylylation by native gel electrophoresis}

For the analysis of GlnK modification, the different mobilities of the uridylylated and unmodified protein in non-denaturating polyacrylamide gels was investigated (Forchhammer and Hedler, 1997). Portein samples were separated by native gel electrohoresis using $12.5 \%$ polyacrylamide gels (29:1, acrylamide:bisacrylamide) with 5\% stacking gels. The buffer for the running gels was $187.5 \mathrm{mM}$ Tris/ $\mathrm{HCl}, \mathrm{pH} \mathrm{8.9,} \mathrm{the} \mathrm{buffer} \mathrm{for} \mathrm{the} \mathrm{stacking} \mathrm{gels} \mathrm{was} 62.5$ $\mathrm{mM}$ Tris/ $\mathrm{HCl}, \mathrm{pH} 7.5$, and the running buffer was $82.6 \mathrm{mM}$ Tris/ $\mathrm{HCl}, \mathrm{pH} 9.4$, containing 33 $\mathrm{mM}$ glycine. After gelelectrophoresis using a BioRad Miniprotein I electrophoresis apparatus and proteins were subsequently transferred on nitrocellulose membranes for western blot analysis. In general, uridylylated forms of GlnK proteins show higher mobilities in nondenaturing polyacrylamide gels resulting in a protein band with an apparent lower molecular mass than the respective non-modified protein.

\section{Results}

In our current working model for the oxygen signal transduction in $K$. pneumoniae, we hypothesize that under anaerobic conditions, the FAD moiety of NifL is reduced by a component of the anaerobic electron transport chain, which is transcriptionally controlled by Fnr. If the reduction of NifL indeed occurs by a membrane-bound electron donor, then NifL must contact the cell membrane. We therefore localized NifL in K. pneumoniae and mutant strains growing under various conditions.

Localization of NifL in $K$. pneumoniae cells by electron microscopy. We localized NifL in K. pneumoniae strain M5a1 grown anaerobically under nitrogen limitation or nitrogensufficient conditions in the presence of $2 \mathrm{mM}$ glutamine or $10 \mathrm{mM}$ ammonium, respectively. The detection of NifL synthezised from the chromosomal nifL gene could not be analyzed statistically by electron microscopy as the level of expression was too low (data not shown). We therefore induced additional NifL expression from the plasmid pNH3 in K. pneumoniae M5a1 with $10 \mu \mathrm{M}$ IPTG to levels at which NifL function is regulated normally in response to oxygen and combined nitrogen in K. pneumoniae (Schmitz et al., 1996). Cells in mid exponential phase grown anaerobically were harvested in the absence of oxygen, and prepared for electron microscopy under a nitrogen atmosphere in a glove box, as described in Materials and Methods. Immunogold detection by electron microscopy analysis of the overexpressed protein in 50 independent cells showed that approximately $76.4 \%$ of total NifL were found in 
close proximity to the cell membrane, when cells were grown under nitrogen-limiting conditions indicating that NifL is membrane associated (Fig. $9 \mathrm{~A}_{1}$ to $\mathrm{A}_{4}$ ). In contrast, in cells grown under nitrogen-sufficient conditions, the NifL protein was, in general, not attached to the cell membrane but was found mainly within the lumen of the cell (up to $80 \%$ of total NifL, Fig. $9 \mathrm{~B}_{1}$ to $\mathrm{B}_{4}$ ). These findings indicate that NifL is apparently membrane associated when synthesized under oxygen- and nitrogen-limitation, but is localized in the cytoplasm when grown in the presence of sufficient nitrogen source.

\section{NifL synthesized from the chromosomal nifL gene is highly membrane associated under} derepressing conditions. Localization of overproduced NifL by electron microscopy indicated that NifL is membrane associated in $K$. pneumoniae when cells are grown anaerobically under nitrogen limitation. As the amount of NifL synthezised from the chromosomal nifL gene was too insignificant for localization by immunogold labelling, we used immunological means for the detection and quantification of NifL synthezised from the chromosomal nifL gene in cytoplasmic and membrane fractions of $K$. pneumoniae cells grown under various conditions. In case of cell extract preparation and separation of membrane and cytoplasmic fraction of anaerobic grown cells all steps were performed in the presence of 2.0 $\mathrm{mM}$ dithiothreitol and under a nitrogen atmosphere.

K. pneumoniae strain UN4495 carrying a chromosomal nifK-lacZ fusion was used for the NifL localization experiments, in order to be able to monitor NifA activity during growth. The cells were grown under nitrogen limitation to induce chromosomal expression of NifL and NifA in the absence or presence of molecular oxygen. In order to control NifL regulation of NifA activity in the respective cultures, we analyzed NifA activity by determining $\beta$ galactosidase activity. In general, anaerobically growing cultures exhibited a $\beta$-galactosidase synthesis rate of approximately $4000 \mathrm{U} \mathrm{ml}^{-1} \cdot \mathrm{OD}_{600}{ }^{-1}$, whereas the synthesis rate of aerobic cultures was determined to be approximately $200 \mathrm{U} \mathrm{ml}^{-1} \cdot \mathrm{OD}_{600}{ }^{-1}$. This indicated that the nif genes were fully induced under nitrogen- and oxygen-limiting conditions, and repressed in the presence of oxygen.

In order to localize NifL in these cells, membranes were prepared under anaerobic or aerobic conditions, and the membrane and cytoplasmic fractions were separated, as described in Materials and Methods. The quality of the membrane preparations was evaluated using malate dehydrogenase as a marker for the cytoplasmic fraction and quinones as a marker for the membrane fraction (see Materials and methods). For the various membrane preparations, we found that, in general, approximately $99 \%$ of the malate dehydrogenase activity was located in the cytoplasmic fraction, and quinones were detectable only in the membrane fraction 
(Table 5). The solubilized proteins of the various membrane and the cytoplasmic fractions were analyzed by gel electrophoresis, and subsequent detection of NifL protein by immunological means. The NifL protein in the different fractions was quantified for each growth condition in three independent membrane preparations using the fluoroimager and the ImageQuant software (Molecular Dynamics). Relative amounts of NifL in the respective fractions relative to total amount were calculated as described in Materials and Methods.
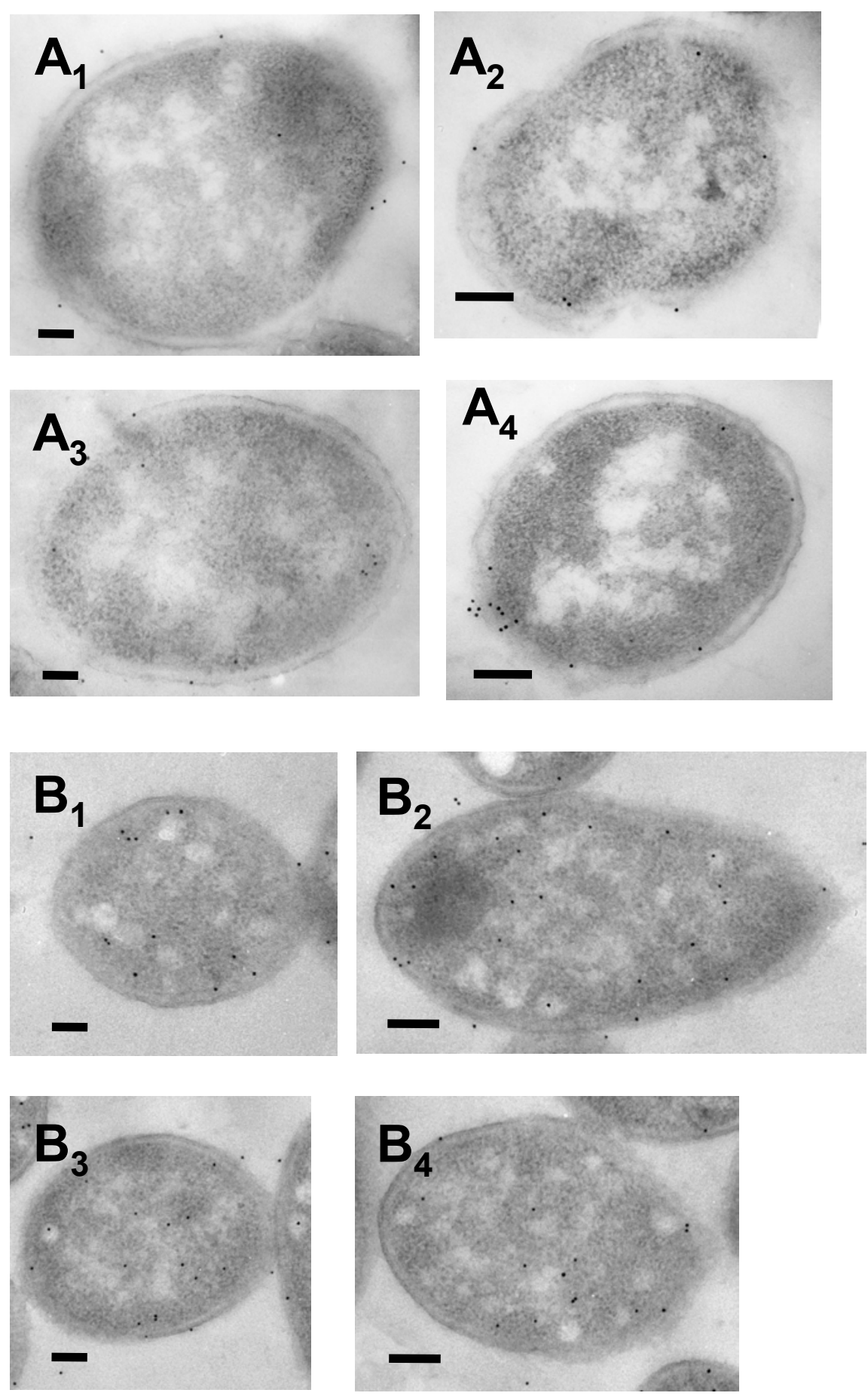
Fig. 9: Effect of ammonium on NifL localization in $K$. pneumoniae. NifL was overexpressed from the tac promoter by $10 \mu \mathrm{M}$ IPTG in $K$. pneumoniae growing anaerobically under nitrogen-limited $\left(\mathrm{A}_{1}\right.$ to $\left.\mathrm{A}_{4}\right)$ and nitrogen excess $\left(\mathrm{B}_{1}\right.$ to $\left.\mathrm{B}_{4}\right)$ conditions. Cells were harvested in mid exponential growth and prepared for electron microscopy as described in Materials and Methods. NifL identified by immunogold labelling appears as dark spots (colloidal gold particles). Horizontal bars equal $0.1 \mu \mathrm{M}$.

Table 5: Quality of membrane preparations. The quality of the membrane preparations of K. pneumoniae UN4495 cells grown under the respective conditions was evaluated by determination of malate dehydrogenase activity according to Bergmayer (1983) and quinoprotein analysis by redox-cycle staining as described by Flückiger et al. (1995) in the respective fractions.

\begin{tabular}{|c|c|c|c|}
\hline Growth condition & Cell fraction & $\begin{array}{l}\text { Malate dehydrogenase } \\
\left.\text { activity (U x fraction }{ }^{-1}\right)\end{array}$ & $\begin{array}{l}\text { Redox cycle stain } \\
\text { (presence of quinoproteins) }\end{array}$ \\
\hline \multirow[t]{2}{*}{ Glutamine, aerobic } & membrane & 0.08 & + \\
\hline & cytoplasm & 22 & - \\
\hline \multirow[t]{2}{*}{ Glutamine, anaerobic } & membrane & 0.1 & + \\
\hline & cytoplasm & 16 & - \\
\hline
\end{tabular}

Initial experiments concentrated on the localization of NifL under nitrogen-limiting conditions, in both the absence and presence of oxygen. Under anaerobic growth conditions, approximately $55 \%$ of total NifL were found in the membrane fraction (Fig. 10, lanes 1 and 2). In contrast, $6 \%$ or less of total NifL synthesized under aerobic growth conditions was found in the membrane fraction (Fig. 10, lanes 5 and 6). The total amount of NifL synthesized under aerobic conditions was in the same range as the total amount synthesized under anaerobic conditions. This indicates that under anaerobic conditions, NifL is membraneassociated, whereas in the presence of oxygen, membrane association of NifL significantly decreases. Next we analyzed anaerobically growing cultures which were shifted from nitrogen-limited growth to nitrogen-excess conditions and grown for an additional $2 \mathrm{~h}$ when examined. Interestingly, although the cells were grown anaerobically, also only approximately $10 \%$ of total NifL was found in the membrane fraction (Fig. 10, lanes 3 and 4). The total amount of NifL, however, did not significantly decrease after the shift to nitrogen sufficiency. Within the $2 \mathrm{~h}$ incubation in the presence of ammonium, no synthesis of NifL can occur because of repression of NifL synthesis by the nitrogen regulatory system (Drummond and Wootton, 1987). Thus, the presence of ammonium apparently resulted in a significant dissociation of NifL from the cytoplasmic membrane. 


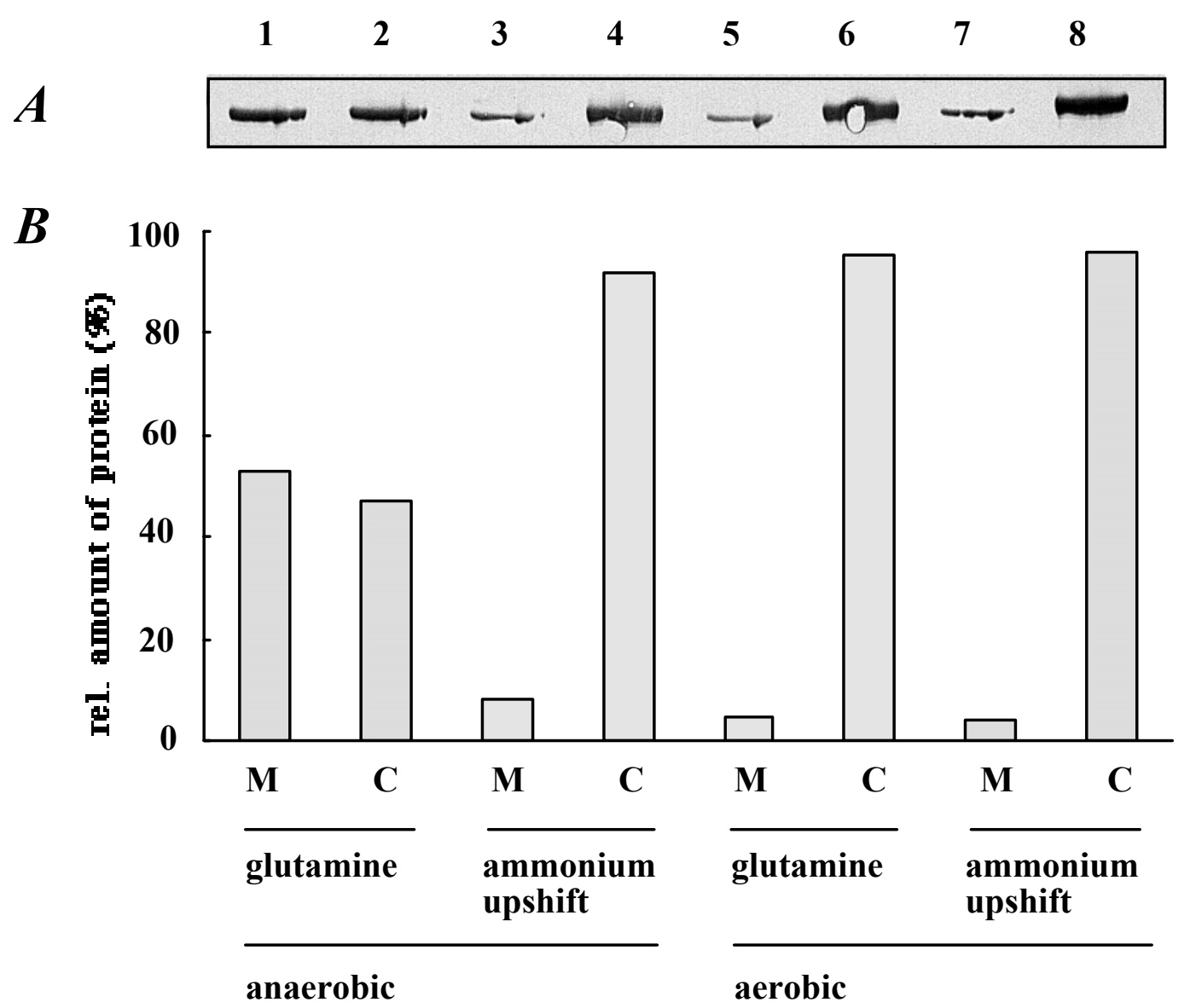

Fig. 10: Localization of NifL synthezised from the chromosomal nifL gene in $K$. pneumoniae UN4495 grown under different conditions. Cells of K. pneumoniae UN4495 were grown aerobically or anaerobically in minimal medium containing $2 \mathrm{mM}$ glutamine as the sole nitrogen source. Exponentially growing cells were split and one half was shifted to ammonium excess $(10 \mathrm{mM})$, as described in Materials and Methods. After an additional $2 \mathrm{~h}$ incubation, the cells were harvested and separated into membrane and cytoplasmic fractions. Aliquots of the observed membrane and cytoplasmic fractions ( $4 \mu 1$ and $20 \mu \mathrm{l}$, respectively) were subjected to SDS-PAGE, and subsequently analyzed by westernblotting. Polyclonal NifL antibodies were used to detect NifL in the fractions. NifL found in the membrane and cytoplasmic fractions was quantified with a fluoroimager (Molecular Dynamics storm, ImageQuant software) as described in Material and Methods. A, original western blot. Lanes 1 and 2, membrane and cytoplasmic fraction of cells grown anaerobically under nitrogen limitation; lanes 3 and 4, membrane and cytoplasmic fraction of cells grown anaerobically under nitrogen limitation but shifted to nitrogen sufficiency and incubated for additional 2 hours; lanes 5 and 6 , membrane and cytoplasmic fraction of cells grown aerobically under nitrogen limitation; lanes 7 and 8 , membrane and cytoplasmic fraction of cells grown aerobically under nitrogen limitation but shifted to nitrogen sufficiency and incubated for additional 2 hours. B, quantity of NifL in the cytoplasmic and membrane fractions, as relative to total NifL; setting the absolute amounts in both fractions of the respective membrane preparation as $100 \%$. 
A

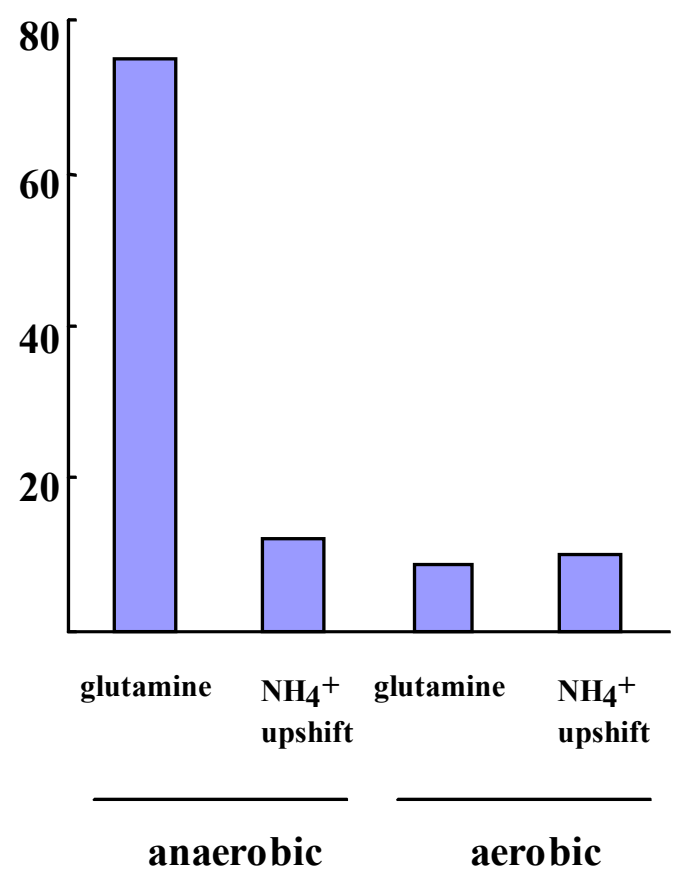

$\boldsymbol{B}$

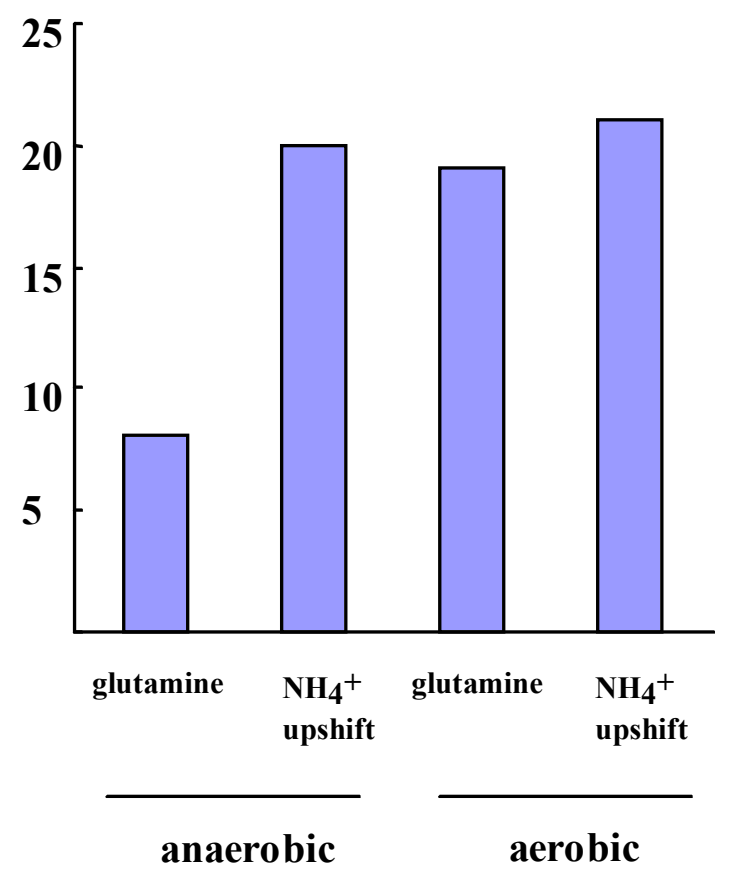

Fig. 11: Comparison of relative amounts of NifL in the membrane fraction (A) and the cytoplasmic fraction (B) of $K$. pneumoniae UN4495 cells grown under different conditions. Total amount of NifL in the respective cell fractions described in Fig. 2 were calculated using a fluoroimager (Molecular Dynamics storm, ImageQuant software) and known amounts of purified NifL-protein. Total amounts of NifL in the membrane (A) and the cytoplasmic fraction (B) under different growth conditions are plotted as relative to total protein in the respective fraction.

This was confirmed by plotting relative amounts of NifL in both the membrane and cytoplasmic fraction as relative to total protein in the respective fraction under the growth conditions tested (Fig. 11). These findings which are consistent with the results obtained by electron microscopy for overproduced NifL (Fig. 9) indicate that NifL is membrane associated only when cells are growing under derepressing nitrogen-fixation conditions. However, both individual signals, molecular oxygen or nitrogen sufficiency appear to result in a significant decrease in the membrane association of NifL to $10 \%$ or less of total NifL. This suggests that the observed spatial separation of membrane-associated NifL and cytoplasmic NifA under anaerobic and nitrogen-limited growth conditions may be responsible for nif gene induction. 


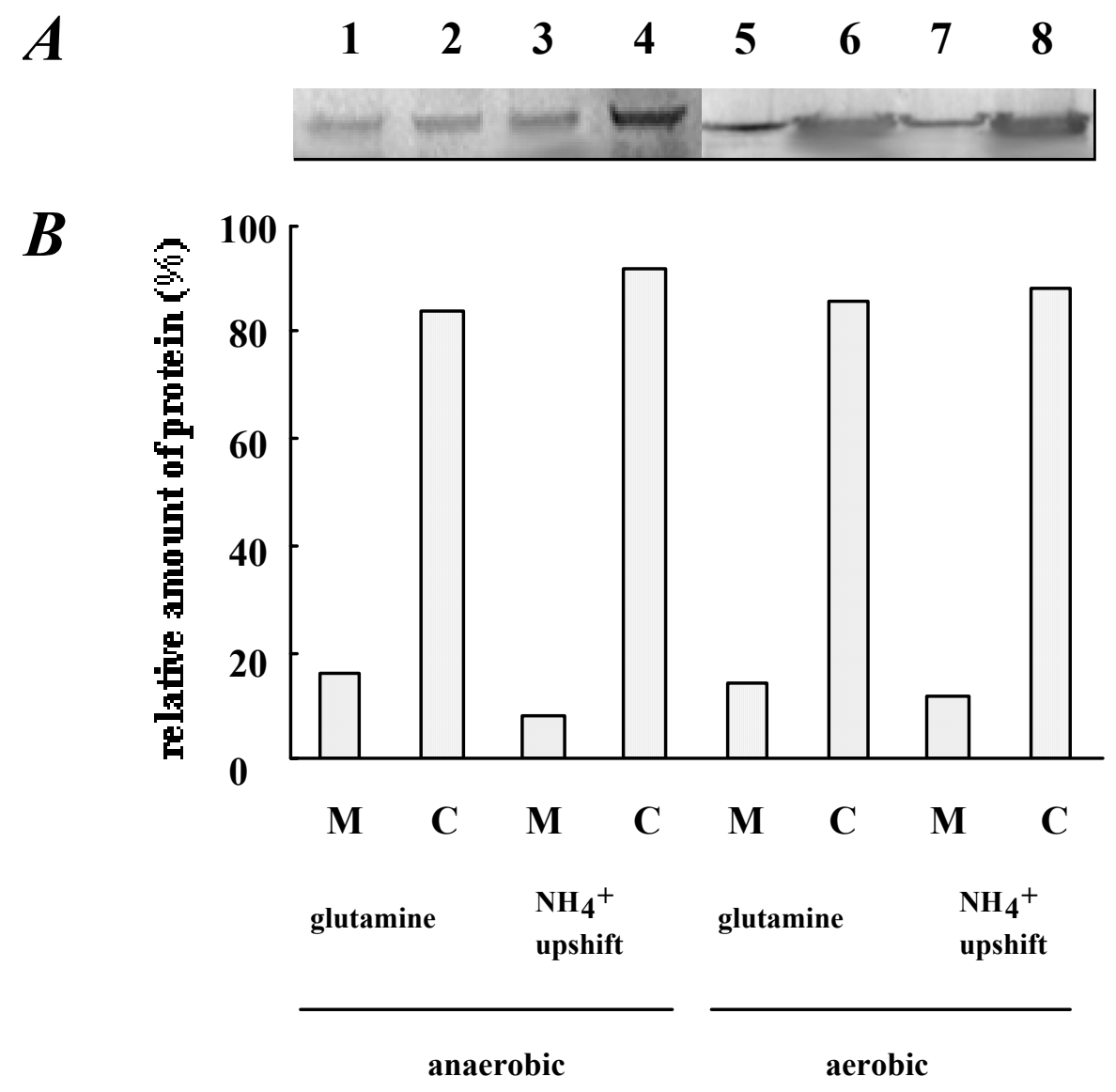

Fig. 12: Localization of NifA synthezised from the chromosomal nifA gene in $K$. pneumoniae UN4495 grown under different conditions. Membrane preparations of $K$. pneumoniae UN4495 cells grown under various conditions were performed as described in the legend of Fig. 2. Aliquots of the observed membrane and cytoplasmic fractions ( $4 \mu 1$ and $20 \mu 1$, respectively) were subjected to SDS-PAGE, and subsequently analyzed by westernblotting. Polyclonal NifA antibodies were used to detect NifA in the fractions. NifA found in the membrane and cytoplasmic fractions was quantified with a fluoroimager (Molecular Dynamics storm, ImageQuant software) as described in Material and Methods. A, original western blot. Lanes 1 and 2, membrane and cytoplasmic fraction of cells grown anaerobically under nitrogen limitation; lanes 3 and 4, membrane and cytoplasmic fraction of cells grown anaerobically under nitrogen limitation but shifted to nitrogen sufficiency and incubated for additional 2 hours; lanes 5 and 6, membrane and cytoplasmic fraction of cells grown aerobically under nitrogen limitation; lanes 7 and 8, membrane and cytoplasmic fraction of cells grown aerobically under nitrogen limitation but shifted to nitrogen sufficiency and incubated for additional 2 hours. B, quantity of NifA in the cytoplasmic and membrane fractions, as relative to total NifA; setting the absolute amounts in both fractions of the respective membrane preparation as $100 \%$.

NifA is located in the cytoplasm under all conditions. In the presence of molecular oxygen or combined nitrogen, NifL inhibits NifA-dependent transcriptional activity by direct proteinprotein interaction. In order to prove the hypothesis of a spatial separation of NifL and its target NifA under oxygen- and nitrogen-limited conditions, we localized NifA synthesized 
from the chromosomal nifLA operon using the same membrane and cytoplasmic fractions in which we had localized NifL. We found that approximately $12 \pm 3 \%$ of total NifA are located in the membrane fraction under all growth conditions tested (Fig. 12). As shown for NifL, no difference in total NifA protein was detected under the various growth conditions. Taking into account that (i) NifA has to be localized in the cytoplasm to activate nif transcription, and (ii) the NifA membrane-associated fraction is under all conditions in the same range as the membrane association of NifL in the presence of oxygen or ammonium, a membrane association in the range of $10 \%$ may be based on non-specific binding of hydrophobic regions of the two proteins to the membrane.

\section{Under derepressing conditions but in the absence of either Fnr or GlnK ant AmtB, NifL}

is located in the cytoplasm. In order to observe additional evidence that both individual signals, molecular oxygen and nitrogen sufficiency, result in a significant decrease in the membrane association of NifL, we localized NifL synthesized from the chromosomal nifL gene under derepressing conditions in the absence of either the oxygen sensory protein Fnr or the nitrogen sensory protein GlnK and AmtB. K. pneumonaie UN4495 carrying an fnr nullallele (K. pneumoniae UN4495 fnr:: $\Omega$, RAS18) and $K$. pneumoniae UN4495 carrying an glnKamtB null-allele ( $K$. pneumoniae UN4495 glnK::KIXX, RAS36) were grown under nitrogen- and oxygen-limited conditions. During growth NifA activity was analyzed by determining $\beta$-galactosidase synthesis rates.

As expected from previous studies (Grabbe et al., 2001b; He et al., 1998; Jack et al., 1999), in the absence of the primary oxygen sensor Fnr (RAS18) or in the absence the primary nitrogen sensor GlnK (RAS36), NifL inhibited NifA activity (Table 6). No obvious differences in the total amounts of NifA or NifL in the mutant backgrounds compared to the parental strain were detected, indicating that the failure of the mutant strains to express nifH under derepressed conditions could not be accounted for by a decreased amount of NifA protein. Localization of NifL in the two mutant strains under nitrogen and oxygen limitation in three independent membrane praparations showed that in both, the $f n r$ mutant and the glnKamtB mutant, approximately $90 \%$ of NifL is localized in the cytoplasmic fraction (Table 6).

Additional shifts to nitrogen excess did not change the NifL location significantly. Thus, in the absence of Fnr or GlnK plus the ammonium transporter AmtB, NifL does not receive the signal for oxygen- or nitrogen limitation, respectively, resulting in a NifL protein which is located in the cytoplasm and inhibits NifA activity. These findings strongly support our model that under derepressing conditions NifL is membrane associated, however either signal, 
molecular oxygen or nitrogen sufficiency, result in a significant decrease of membrane association of NifL.

Table 6: Localization of NifL synthezised from the chromosomal nifL gene in $K$. pneumniae UN4495 and derived mutants under oxygen and nitrogen limitation and after a shift to nitrogen sufficiency. nif induction was monitored by measuring the differential rates of $\beta$-galactosidase synthesis as described by Schmitz et al. (1996). Membrane and cytoplasmic fractions were separated and NifL was immunological quantified in the cytoplasmic and membrane fractions by western-blot analysis using a fluoroimager (Molecular Dynamics storm, ImageQuant software) and purified proteins as desribed in Methods. Cells were grown under nitrogen-limitation in the presence of $4 \mathrm{mM}$ glutamine (nitrogen -limitation) or were shifted to ammonium excess und further incubated for $2 \mathrm{~h}$ (ammonium upshift). The relative amount of NifL in the respective fraction is presented in $\%$ of total NifL and the absolute amount of NifL in $\mu \mathrm{g}$ NifL per mg protein of the respective cell fraction. Data presented represent mean values of three independent experiments.

\begin{tabular}{|c|c|c|c|c|c|c|}
\hline Strain & $\begin{array}{r}\mathrm{UN} \\
(\varnothing(n i f K-l \\
4001 \mathrm{hi}\end{array}$ & $\begin{array}{l}4495 \\
c Z) 5935 \Delta l a c- \\
\left.D 4226 \mathrm{Gal}^{\mathrm{r}}\right)\end{array}$ & \multicolumn{2}{|c|}{$\begin{array}{l}\text { UN4495 fnr:: } \Omega \\
\text { (RAS18) }\end{array}$} & \multicolumn{2}{|c|}{$\begin{array}{c}\mathrm{UN} 4495 g \ln K: \mathrm{KIXX} \\
(\mathrm{RAS} 36)\end{array}$} \\
\hline $\begin{array}{l}\text { Expression of } \\
\text { nifH'-'lacZ } \\
\left(\mathrm{U} \cdot \mathrm{ml}^{-1} \cdot \mathrm{OD}_{600}{ }^{-1}\right)\end{array}$ & \multicolumn{2}{|c|}{$4000 \pm 100$} & \multicolumn{2}{|c|}{$250 \pm 20$} & \multicolumn{2}{|c|}{$\leq 10$} \\
\hline cell fraction & membran & cytoplasm & membrane & cytoplasm & membrane & cytoplasm \\
\hline $\begin{array}{c}\text { Nitrogen-limitation } \\
(\%)\end{array}$ & $55 \pm 5$ & $45 \pm 5$ & $10 \pm 3$ & $90 \pm 3$ & $13 \pm 2$ & $87 \pm 2$ \\
\hline $\begin{array}{l}\text { Nitrogen-limitation } \\
(\mu \mathrm{g} \mathrm{NifL/mg} \\
\text { fraction protein) }\end{array}$ & $75 \pm 4$ & $8 \pm 1$ & $25 \pm 3$ & $22 \pm 3$ & $27 \pm 3$ & $23 \pm 3$ \\
\hline $\begin{array}{c}\text { Ammonium-upshift } \\
(\%)\end{array}$ & $7 \pm 3$ & $93 \pm 3$ & $6 \pm 2$ & $94 \pm 3$ & $10 \pm 3$ & $90 \pm 3$ \\
\hline $\begin{array}{l}\text { Ammonium-upshift } \\
\text { ( } \mu \mathrm{g} \mathrm{NifL/mg} \\
\text { fraction protein) }\end{array}$ & $18 \pm 2$ & $22 \pm 3$ & $24 \pm 2$ & $21 \pm 2$ & $25 \pm 3$ & $22 \pm 2$ \\
\hline
\end{tabular}




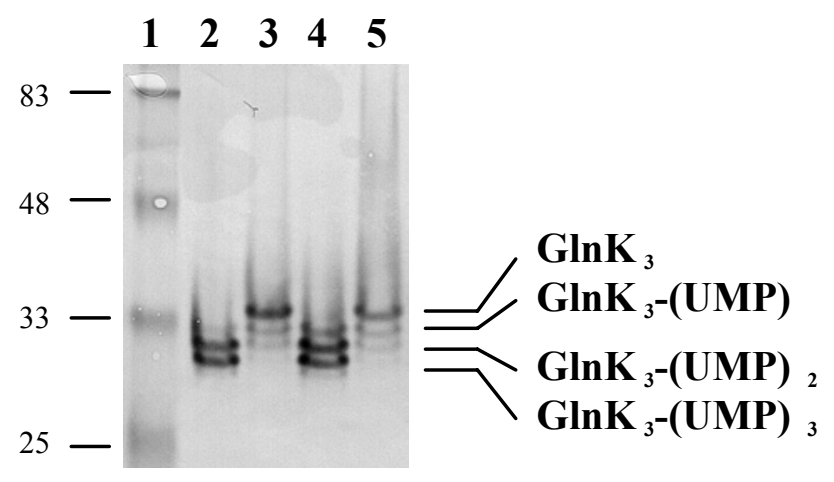

Fig. 13: Uridylylation states of GInK upon an ammonium upshift. $K$. pneumoniae wild type cultures were grown aerobically and anaerobically in the presence of $2 \mathrm{mM}$ glutamine as sole nitrogen source as described in Materials and Methods. During mid-exponential growth, cultures were split and one part was shifted to nitrogenexcess conditions by the addition of $10 \mathrm{mM}$ ammonium. After an additional incubation of 2 hours the cells were harvested, broken by French Press and analyzed by native-PAGE. Subsequent western-blotting using polyclonal GlnK antibodies was used to detect uridylylated and unmodified GlnK synthesized from the chromosomal $g \ln K$ gene. Lane 1, broad range prestained marker (New England Biolabs); lane 2, cell extract of anaerobically grown cells in the presence of $2 \mathrm{mM}$ glutamine; lane 3, cell extract of anaerobically grown cells after an ammonium upshift with $10 \mathrm{mM}$ ammonium; lane 4, cell extract of aerobically grown cells in the presence of $2 \mathrm{mM}$ glutamine, and lane 5, cell extract of aerobically grown cells after an ammonium upshift with $10 \mathrm{mM}$ ammonium.

Under nitrogen limitation GInK is partially membrane associated independent of the oxygen status. NifL is membrane associated under oxygen- and nitrogen-limited conditions and dissociates from the membrane upon a shift to nitrogen sufficiency (Figs. 10 and 11). Thus the question arises, how does the NifL/NifA regulatory system receive the nitrogen signal when an upshift to nitrogen sufficiency occurs. As the GlnK protein apparently senses the nitrogen status of the cell and transduces the nitrogen signal to the NifL/NifA regulatory system, GlnK was localized under nitrogen-limiting conditions and after a shift to excess nitrogen.

In $K$. pneumoniae, the $g \ln K$ gene, a $g \ln B$-like gene, is under the control of the general nitrogen regulatory system, and therefore only expressed under nitrogen starvation as is $E$. coli glnK (van Heeswjik et al. 1996; Jack et al., 1999; Arcondéguy et al., 2001). In response to nitrogen limitation, the trimeric E. coli $\mathrm{GlnK}$ protein is covalently modified by uridylylation at the conserved tyrosine residue (Y51) by the GlnD enzyme. In the presence of ammonium, however, GlnD removes the uridylylation (Atkinson and Ninfa, 1999; Jiang et al., 1998). In K. pneumoniae cells grown either anaerobically or aerobicically under nitrogen- 
limiting conditions, GlnK trimers were up to $80 \%$ uridylylated $\left(\mathrm{GlnK}_{3}-(\mathrm{UMP})_{3}\right)$ as detected by native gel electrophoresis and subsequent western blot analysis (Fig. 13, lanes 2 and 4); the uridylylation apparently changes the overall charge of the trimers resulting in a faster migration of the uridylylated forms compared to the non-modified trimers. Two hours after an ammonium upshift, the same cultures showed fully deuridylylated GlnK trimers (Fig. 13, compare lanes 2 and 4 with lanes 3 and 5). These findings show that the uridylylation state of $K$. pneumoniae GlnK, like that of $E$. coli GlnK, is dependent on the nitrogen status of the cell. In order to analyze and localize GlnK trimers after a shift to nitrogen sufficiency, we performed ammonium upshift experiments on $K$. pneumoniae cells grown under nitrogenlimited conditions in the presence or absence of oxygen. Exponentially growing cultures were split and one part was shifted to nitrogen sufficiency by the addition of $10 \mathrm{mM}$ ammonium and further incubated for $2 \mathrm{~h}$. The $g \ln \operatorname{KamtB}$ operon is subject to nitrogen control at the transcriptional level mediated by NtrC (Jack et al., 1999), thus within the $2 \mathrm{~h}$ incubation in the presence of ammonium, no expression of $g \ln K$ can occur. The membrane and cytoplasmic fractions before and after the ammonium upshift were subjected to native PAGE and subsequent western blot analysis to separate and quantify the GlnK trimers in the different fractions. In the cell-free extracts under nitrogen-limiting conditions approximately $80 \%$ GlnK was found in its completely uridylylated form $\left(\mathrm{GlnK}_{3}-(\mathrm{UMP})_{3}\right) .15$ to $20 \%$ of the total GlnK protein was found in the membrane fraction, in both the anaerobic and the aerobic preparation (Fig. 14B, lanes 1 and 2, lanes 5 and 6). The membrane-bound GlnK and cytoplasmic GlnK, however, showed no difference in the uridylylation pattern, indicating that membrane association is not dependent on a defined uridylylation state of GlnK (Fig. 14A, lanes 1 and 2, lanes 5 and 6). This observed membrane association of GlnK under nitrogen limitation is of special interest, since the GlnK protein shows little if any hydrophobicity, and is a highly soluble protein. 


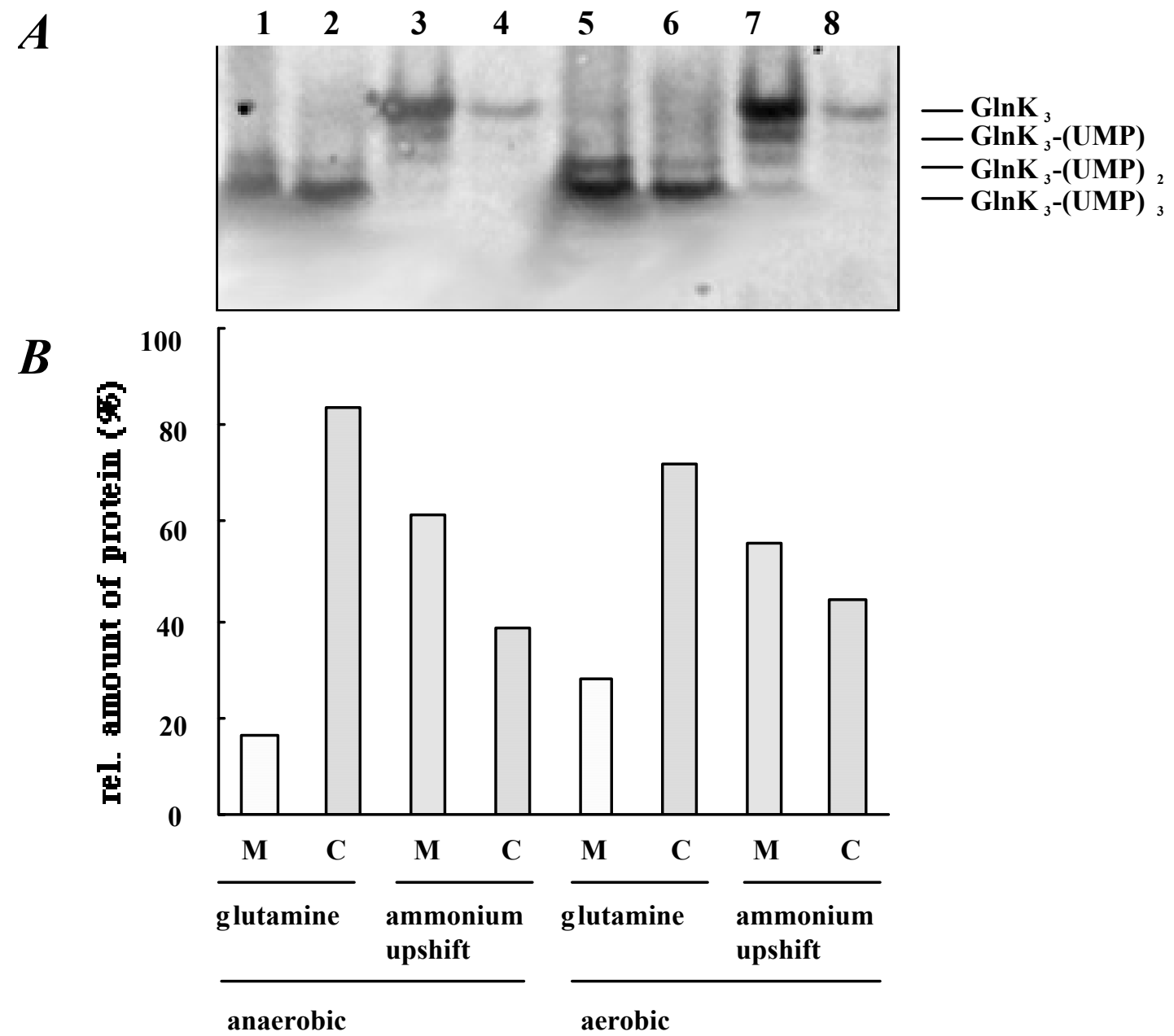

Fig. 14: Localization of GInK in $K$. pneumoniae UN4495 grown under different conditions. $K$. pneumoniae UN4495 cells were grown, harvested and fractionated as described in Fig. 2. Equal volumes of the observed membrane and cytoplasmic fractions ( $20 \mu \mathrm{l}$ ) were subjected to native PAGE and subsequently analyzed by western-blotting. Polyclonal GlnK antibodies were used to detect GlnK. The western blot is shown in A. Lanes 1 and 2, membrane and cytoplasmic fraction of cells grown anaerobically under nitrogen limitation; lanes 3 and 4 , membrane and cytoplasmic fraction of cells grown anaerobically under nitrogen limitation but shifted to nitrogen sufficiency and incubated for an additional 2 hours; lanes 5 and 6, membrane and cytoplasmic fraction of cells grown aerobically under nitrogen limitation; lanes 7 and 8, membrane and cytoplasmic fraction of cells grown aerobically under nitrogen limitation but shifted to nitrogen sufficiency and incubated for additional 2 hours. The amounts of GlnK found in the membrane and cytoplasmic fractions were quantified using a fluoroimager (Molecular Dynamics storm, ImageQuant software), concentrations of GlnK were corrected for fraction volume (total volume of the membrane fraction was $2 \mathrm{ml}$; total volume of the cytoplasmic fraction was $10 \mathrm{ml}$ ), and plotted as relative amounts of total GlnK in the respective fraction, in $\mathbf{B}$.

When nitrogen limited $K$. pneumoniae cells were shifted from nitrogen limitation to nitrogen excess, GlnK in both the membrane and the cytoplasmic fractions was deuridylylated (Fig. 
14A, lanes 3 and 4, lanes 7 and 8). However, it appeared that after a shift to nitrogen-excess independent of oxygen availability the cytoplasmic GlnK fraction decreased significantly. In contrast, the amount of membrane-associated GlnK apparently did not change (Fig. 14A, compare lanes 2 and 4, and lanes 6 and 8), thus shifting the ratio of membrane-associated GlnK to cytoplasmic GlnK from $15 \%$ / $85 \%$ under nitrogen limitation, to approximately 60 $\% / 40 \%$ in the presence of excess nitrogen (Fig. 14B). As no new GlnK synthesis occurred during the ammonium upshift the ratio between membrane-bound and cytoplasmic GlnK should not change unless increased degradation of one fraction occurs. However, one cannot rule out that unspecific proteolysis of the cytoplasmic GlnK fraction occurred during the time of separating the two fractions, as the buffers used for cell breakage and membrane preparation were not supplemented with protease inhibitors. Thus, further analysis of the apparent faster degradation of cytoplasmic GlnK after a shift to nitrogen sufficiency is required.

\section{Discussion}

Regulatory proteins that are membrane bound and transmit an environmental signal via a cytoplasmic transmitter domain are a common principle in bacterial signal transduction. In a variety of such regulatory proteins or transducers of both prokaryotic and eukaryotic origin, conserved sequence motifs, so called PAS domains, have been identified (for review see Taylor and Zhulin, 1999). Most bacterial sensory proteins containing a PAS domain are histidine kinase sensor proteins of two-component regulatory systems, and usually contain one or more transmembrane domains (Zhulin et al., 1997; Taylor and Zhulin, 1999). The regulatory protein NifL contains a C-terminal histidine-kinase-like transmitter domain (Drummond and Wootton, 1987; Parkinson and Kofoid, 1992; Woodley and Drummond, 1994) and its N-terminal domain contains the conserved motifs of the PAS domain (Zhulin et al., 1997). NifL differs, however, in that no membrane-spanning domain can be predicted from amino acid sequence data of the protein (Drummond and Wootton, 1987). Thus, NifL is considered to be a solely cytoplasmic protein that receives and transduces the oxygen and nitrogen signal to the transcriptional activator NifA in the cytoplasm (Dixon, 1998). We have recently shown that Fnr of $K$. pneumoniae is the primary oxygen sensor for nitrogen fixation, which apparently transduces the oxygen signal to NifL by activating transcription of genes, whose products reduce the NifL-bound FAD under anaerobic conditions (Grabbe et al., 2001b). In addition, preliminary studies indicated that $K$. pneumoniae NifL is membrane- 
associated during anaerobic growth. Thus we proposed that the physiological electron donor for the reduction of NifL during anaerobic growth is a component of the anaerobic electron transport chain. In order to characterize a potential membrane association of NifL as a part of the regulatory process further, we localized NifL in cells grown anaerobically and aerobically, both in the absence and presence of combined nitrogen.

\section{Spatial separation as the potential regulatory principle in nif regulation by NifA and}

NifL. We present three lines of evidence that both conditions, nitrogen limitation and the absence of oxygen, are required for significant membrane association of $K$. pneumoniae NifL. Either signal alone is not sufficient for NifL association with the membrane. (i) Electron microscopy analysis of NifL overproduced in K. pneumoniae indicated that under oxygenand nitrogen-limiting conditions, NifL is significantly membrane associated, whereas under nitrogen sufficiency NifL is located in the cell lumen (Fig. 9). (ii) Immunological quantifications of NifL synthesized from the chromosomal nifL gene confirmed that under oxygen and nitrogen limitation, approximately $55 \%$ of the total NifL protein is located in the membrane fraction. A shift to nitrogen sufficiency or the presence of molecular oxygen, however, resulted in a significant decrease in membrane association of NifL, to approximately $10 \%$ (Fig. 10). (iii) In the absence of eiher the primary oxygen sensor Fnr or the primary nitrogen sensor GlnK plus the ammonium transporter AmtB, NifL is located in the cytoplasm (Table 6). Thus, in addition to nitrogen limitation, the reduced conformation of NifL appears to be critically important for the membrane affinity of the protein. With oxidation, the membrane affinity of NifL significantly decreases, and NifL is again located in the cytoplasm. Determination of malate dehydrogenase activity and detection of quinoproteins in the different membrane and cytoplasmic fractions ruled out that the analyzed membrane fractions were contaminated with cytoplasmic proteins (Table 5). Thus, the basal amount of a maximum of $10 \%$ membrane-bound NifL, detected under all conditions except under oxygen- and nitrogen-limitation, appears to be based on non-specific binding of the hydrophobic regions of the NifL protein to the cell membrane. This is consistent with the amounts we observed for the NifA protein in the same membrane and cytoplasmic fractions; approximately $10 \%$ of total NifA were membrane associated under all conditions tested (Fig. 12), although NifA is a transcriptional activator and is therefore expected to be a soluble protein located in the cytoplasm (Austin et al., 1990; Lee et al., 1993). This suggests that the observed fractions of NifA and NifL, which appear to be membrane associated under all conditions, are fractions of both regulatory proteins, which bind, independent from each other, non-specifically to the membrane and are not functionally involved in the regulatory process. 
The observed decrease in cytoplasmic NifL under anaerobic and nitrogen-limited conditions, conditions under which no change of NifA location is detectable, suggests that membrane association of NifL plays a critical role in the regulation of NifA activity. The spatial separation of membrane-bound NifL and cytoplasmic NifA under nitrogen and oxygen limitation may be responsible for the release of the NifL inhibition of NifA resulting in nif gene induction. We therefore propose a NifL conformation that integrates the oxygen and nitrogen signal in such a way that the overall conformation of the protein under anaerobic and nitrogen-limited conditions is able to bind to the cytoplasmic membrane, creating a spatial gap between NifL and its target NifA. A comparable regulatory mechanism is discussed for the transcriptional regulator PutA, which is involved in proline catabolism in Salmonella typhimurium and Escherichia coli (Maloy, 1987). PutA associates with the membrane and catalyzes the two-step oxidation of proline to glutamate when the intracellular proline concentration is high (Muro-Pastor et al., 1997; Wood, 1987); when the intracellular proline concentration decreases, PutA dissociates from the membrane and represses transcription of the proline utilization ( $p u t$ ) operon by binding to an operator (Ostrovsky et al., 1991; Brown and Wood, 1993). In contrast to the observed membrane affinity of NifL in K. pneumoniae under oxygen and nitrogen limitation, no membrane association for $A$. vinelandii NifL has been reported to date (Dixon, 1998).

\section{Hypothetical function for GInK in nif regulation}

Concerning the nif regulation by combined nitrogen, we observed evidence that a shift from nitrogen limitation to nitrogen sufficiency results in a decrease in membrane association of NifL (Figs. 9 and 10). Thus the question arises, how does the presence of sufficient nitrogen change the membrane affinity of NifL. We therefore localized GlnK, a highly soluble protein, which is responsible for the detection of the internal nitrogen status and for the transduction of the nitrogen signal to the nif regulatory system (Xu et al., 1998, He, et al., 1998; Jack et al., 1999; Arcondéguy et al., 1999 and 2001). Unexpected, we observed a significant membrane association of GlnK under nitrogen-limiting conditions (approximately 15 - 20 \%, Fig. 14), which may result from its interaction with the ammonium transporter AmtB and is not dependent on a defined uridylylation state of GlnK. At the actual experimental status however, one cannot decide if the observed membrane-association of GlnK is directly linked to the NifL location or whether GlnK is regulating the NifL location indirectly as a consequence of its role in controlling the interaction of NifL with NifA. Under nitrogen excess in the absence of GlnK, cytoplasmic NifL inhibits NifA by complex formation. If 
GlnK transduces the signal of nitrogen limitation either by interacting with NifL or NifA resulting in the dissociation of the NifL/NifA complex, NifL would be able to interact with the putative membrane bound electron donor and stays membrane-associated. In the presence of oxygen however, the oxidized form of NifL would be preferentially located in the cytoplasm and this conformation may interact with NifA even when GlnK is present. 


\title{
Chapter 5:
}

\section{Oxygen Control of nif Gene Expression in Klebsiella pneumoniae is dependent on NifL reduction at the cytoplasmic membrane by}

\author{
electrons derived from the reduced quinone pool
}

\section{ROMAN GRABBE AND RUTH A. SCHMITZ*}

\begin{abstract}
In Klebsiella pneumoniae, NifA mediated transcriptional activation of the nitrogen fixation (nif) genes is inhibited in the presence of molecular oxygen by the negative regulator NifL. The primary oxygen sensor Fnr transduces the signal of anaerobiosis to the negative regulator resulting in the non-inhibitory, reduced conformation of the flavoprotein NifL. We have recently demonstrated that membrane sequestration of NifL under anaerobic and nitrogenlimited conditions impairs inhibition of cytoplasmic NifA by NifL and thus seems to be involved in the regulatory mechanism for oxygen dependent nif-regulation in K. pneumoniae. We have now investigated the influence of different membrane-bound oxidoreductases of the anaerobic electron transport chain on nif-regulation in K. pneumoniae by biochemical analysis of purified NifL and by monitoring NifA-mediated expression of nifH'-'lacZ reporter fusions in different genetic backgrounds. In vitro analysis showed that NifL-bound FAD-cofactor was reduced by $\mathrm{NADH} / \mathrm{H}^{+}$only in the presence of either a redox mediator or anaerobic inside-out vesicles derived from anaerobically grown $K$. pneumoniae cells, indicating that in vivo NifL is reduced by a membrane-bound component of the anaerobic electron transport chain. This mechanism is further supported by three lines of evidence: First, Klebsiella strains carrying null mutations of $f d n G$ or nuoCD showed significantly reduced nif induction under derepressing conditions, indicating that NifL inhibition of NifA was not relieved in the absence of formate dehydrogenaseN or NADH:ubiquinone oxidoreductase. The same effect was observed in a heterologous $E$. coli system carrying a $n d h$ null allele (coding for NADH dehydrogenase II). Second, studying nif induction in K. pneumoniae under different growth conditions revealed that the presence of nitrate during anaerobic growth on glycerol under nitrogen limitation resulted in a significant decrease of nif induction. However, when growing
\end{abstract}


on sucrose or glucose, nitrate did not effect nif regulation. The final line of evidence is that a reduced quinone derivative, dimethylnaphthoquinone ${ }_{r e d}\left(\mathrm{DMN}_{\mathrm{red}}\right)$ is able to transfer electrons to the FAD-moiety of purified NifL resulting in the reduced conformation of NifL. On the basis of these data, we postulate that under anaerobic and nitrogen-limiting conditions NifL inhibition on NifA activity is relieved by reduction of the FAD-cofactor at the cytoplasmic membrane through the reduced quinone pool of the anaerobic electron transport chain.

\section{INTRODUCTION}

In the free-living diazotroph Klebsiella pneumoniae, a member of the $\gamma$-subgroup of proteobacteria, nitrogen $\left(\mathrm{N}_{2}\right)$ fixation is tightly controlled to avoid unnecessary consumption of energy. The transcriptional activator NifA and the inhibitor NifL, both under the control of the $\mathrm{NtrB} / \mathrm{C}$-system, regulate the transcription of the nitrogen fixation (nif) operons according to the environmental signals molecular oxygen and combined nitrogen (for review see Dixon 1998, Schmitz et al. 2002). Under oxygen and nitrogen limitation the inhibitor NifL stays in the non-inhibitory conformation and nif-gene expression is activated by NifA. In the presence of oxygen or combined nitrogen, NifL antagonizes the activity of NifA resulting in a decrease of nif-gene expression. The translationally coupled synthesis of nifL and nifA in addition to evidence from immunological studies of complex formation, imply that the inhibition of NifA activity by NifL occurs via a direct protein-protein interaction (Govantes et al. 1998; Henderson et al. 1989). Recently, in the diazotroph Azotobacter vinelandii formation of NifL/NifA complexes has been demonstrated by in vitro co-chromatography in the presence of adenine nucleotides and using the yeast-two-hybrid system (Money et al. 1999 and 2001, Lei et al. 1999).

Recent studies revealed that the nitrogen signal in $K$. pneumoniae and A. vinelandii is transduced towards the regulatory proteins NifL and NifA by the GlnK protein, a paralogue PII-protein. However, the mechanism appears to be opposite in K. pneumoniae and $A$. vinelandii. In K. pneumoniae, relief of NifL inhibition under nitrogen limiting conditions depends on the presence of GlnK, the uridylyation state of which appears not to be essential for its nitrogen signaling function (He et al. 1998, Jack et al. 1999, Arcondeguy et al. 1999 and 2000). It is currently not known, whether GlnK interacts with NifL or NifA alone or affects the NifL/NifA-complex in $K$. pneumoniae. In contrast to $K$. pneumoniae, nonuridylylated GlnK protein appears to activate the inhibitory function of $A$. vinelandii NifL under nitrogen excess, whereas under nitrogen limitation the inhibitory activity of NifL is 
apparently relieved by elevated levels of 2-oxoglutarate (Little et al. 2000, Reyes-Ramirez et al. 2001). Very recently interactions between $A$. vinelandii GlnK and NifL was demonstrated using the yeast-two-hybrid system and in vitro studies further indicated that the nonuridylylated form of $A$. vinelandii GlnK directly interacts with NifL preventing nif-gene expression (Little et al. 2002, Rudnick et al. 2002).

For the oxygen-signaling pathway it was shown that $A$. vinelandii NifL and $K$. pneumoniae NifL act as redox-sensitive regulatory proteins. NifL modulates NifA activity in response to the redox-state of its N-terminal bound FAD-cofactor and allows NifA activity only in the absence of molecular oxygen, when the flavin cofactor is reduced (Hill et al. 1996, Schmitz 1997, Dixon 1998, Macheroux et al. 1998, Klopprogge and Schmitz 1999). Thus, under anaerobic conditions in the absence of combined nitrogen, reduction of the flavin moiety of NifL results in a non-inhibitory conformation of the NifL protein. Recently, we have demonstrated that in K. pneumoniae the global regulator Fnr is required to mediate the signal of anaerobiosis to NifL (Grabbe et al. 2001b). Thus, we proposed that in the absence of oxygen the primary oxygen sensor Fnr activates transcription of gene(s) the product(s) of which reduce the NifL-bound FAD-cofactor resulting in a non-inhibitory conformation of NifL, which allows NifA activity. Further localization analyses of NifL under various growth conditions showed that only under derepressing conditions NifL is highly membraneassociated impairing the inhibition of cytoplasmic NifA. This indicates that sequestration of NifL to the membrane under anaerobic and nitrogen-limited conditions is involved in the regulation of NifA activity by NifL (Klopprogge et al. 2002). Based on these findings the question arises, whether NifL reduction occurs at the cytoplasmic membrane by a component of the anaerobic electron transport chain during membrane association of NifL. In order to verify this hypothesis and to identify the electron donor - potentially localized in the cytoplasmic membrane - we analyzed the effects of different membrane-bound oxidoreductases of the anaerobic electron transport chain on nif-regulation in K. pneumoniae and in a heterologous $E$. coli system. In addition in vitro reduction of purified NifL was studied using artificial electron donors or $\mathrm{NADH} / \mathrm{H}^{+}$in the presence of inverted vesicles derived from K. pneumoniae cells. 


\section{MATERIAL AND METHODS}

\section{Bacterial strains and Plasmids}

The bacterial strains and plasmids used in this study are listed in Table 7. Plasmid DNA was transformed into E. coli cells according to the method of Inoue et al. (1990) and into $K$. pneumoniae cells by electroporation. Transduction by phage P1 was performed as described previously (Silhavy et al. 1984).

Table 1. Bacterial strains and plasmids used in this study

\begin{tabular}{|c|c|c|}
\hline Strain or plasmid & Relevant genotype & Source, reference \\
\hline \multicolumn{3}{|l|}{ Strains: } \\
\hline \multicolumn{3}{|l|}{$\begin{array}{l}\text { Klebisella } \\
\text { pneumoniae: }\end{array}$} \\
\hline M5al & Wild type & MacNeil et al. 1981 \\
\hline UN4495 & $\phi($ nifK-lacZ)5935 $\Delta l a c-4001$ his D4226 Gal & MacNeil et al. 1981 \\
\hline RAS 18 & UN4495, but $f n r:: \Omega$ & Grabbe et al. 2001 \\
\hline RAS46 & $\begin{array}{l}\text { UN4495, but spontaneous streptomycine } \\
\text { resistance }\end{array}$ & This study \\
\hline RAS47 & UN4495, but nuoCD::tet & This study \\
\hline RAS48 & UN4495, but $f d n G::$ tet & This study \\
\hline RAS49 & UN4495, but frdA::tet & This study \\
\hline \multicolumn{3}{|l|}{ E. coli: } \\
\hline NCM1529 & 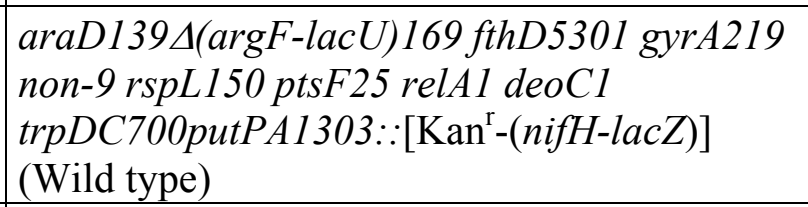 & He et al. 1998 \\
\hline NCM1528 & NCM1529/pNH3 & He et al. 1998 \\
\hline NCM1527 & NCM1529/pJES851 & He et al. 1998 \\
\hline RAS50 & NCM1529, but $n d h::$ tet & This study \\
\hline RAS51 & RAS50 + pNH3 & This study \\
\hline RAS52 & RAS50 + pJES851 & This study \\
\hline RAS53 & NCM1529, but frd::tet & This study \\
\hline RAS54 & RAS53/pNH3 & This study \\
\hline RAS55 & RAS53/pJES851 & This study \\
\hline \multicolumn{3}{|l|}{ Plasmids: } \\
\hline $\mathrm{pBSK}^{+}$ & cloning vector & Stratagene \\
\hline pCR 2.1 & Topo-TA cloning vector & Invitrogen \\
\hline pKAS46 & $\begin{array}{l}\text { allelic exchange vector, oriR6K; } \\
\operatorname{rps}^{*}\left(\operatorname{Strep}^{\mathrm{s}}\right), \mathrm{Amp}^{\mathrm{r}}, \mathrm{Kan}^{\mathrm{r}}\end{array}$ & \begin{tabular}{|l|} 
Skorupsky K. \& \\
R.K. Taylor, 1996
\end{tabular} \\
\hline $\mathrm{pNH3}$ & $\begin{array}{l}\text { K. pneumoniae nifLA under the control of the } \\
\text { tac promoter }\end{array}$ & $\begin{array}{l}\text { Henderson et al. } \\
1989\end{array}$ \\
\hline pJES851 & $\begin{array}{l}\text { K. pneumoniae nifA under the control of tac } \\
\text { promoter }\end{array}$ & Schmitz et al. 1996 \\
\hline pJES794 & $\begin{array}{l}\text { K. pneumoniae malE-nifL under the control of } \\
\text { the tac promoter }\end{array}$ & $\begin{array}{l}\text { Narberhaus et al. } \\
1995\end{array}$ \\
\hline
\end{tabular}




\begin{tabular}{|l|l|l|}
\hline & & \\
\hline pRS167 & $\begin{array}{l}\text { EcoRI/HindIII } f d n G \text { fragment }(\text { K. pneumoniae } \\
\text { M5a1) in pBSK }\end{array}$ & This study \\
\hline pRS177 & pRS167, but $f d n G::$ tet & This study \\
\hline pRS187 & $\begin{array}{l}\text { frdA fragment }(\text { K. pneumoniae M5a1) in } \\
\text { pCR2.1 }\end{array}$ & This study \\
\hline pRS191 & $\begin{array}{l}\text { EcoRI/HindIII nuoCD fragment }(K . \\
\text { pneumoniae M5a1) in pBSK }^{+}\end{array}$ & This study \\
\hline pRS193 & fdnG::tet fragment from pRS177 in pKAS46 & This study \\
\hline pRS194 & pRS191, but nuoCD : tet & This study \\
\hline pRS197 & nuoCD::tet fragment from pRS194 in pKAS46 & This study \\
\hline pRS214 & pRS187, but frdA::tet & This study \\
\hline pRS215 & frdA::tet fragment from pRS214 in pKAS46 & This study \\
\hline
\end{tabular}

(i) E. coli strains:

E. coli NCM1529, containing a chromosomal nifH'-lac $Z^{\prime}$ fusion (He et al. 1997) was chosen to study NifA and NifL regulation in E. coli. The ndhII::tet allele was transferred from ANN001 (T. Friedrich, unpublished) into NCM1529 by P1 mediated transduction with selection for tetracycline resistance, resulting in RAS50. Strains RAS51 and RAS52 contain plasmid pNH3 and plasmid pJES851, respectively.

(ii) K. pneumoniae strains:

K. pneumoniae strain M5al (wild type, $\mathrm{N}_{2}$-fixing) and strain UN4495 [ $\phi$ (nifK-lacZ) 5935 $\Delta l a c-4001$ his D4226 Gal ${ }^{r}$ ] (McNeill et al. 1981) were provided by Gary Roberts. The spontaneous streptomycin resistant UN4495 strain, RAS46, carrying a rpsL mutation was isolated by plating UN4495 on a Luria-Bertani (LB) agar plate containing $100 \mu \mathrm{g}$ streptomycin per ml. K. pneumoniae subsp. pneumoniae (DSM No. 4799, not $\mathrm{N}_{2}$-fixing) and K. oxytoca (DSM No. 4798, not $\mathrm{N}_{2}$-fixing) were obtained from the Deutsche Sammlung von Mikroorganismen und Zellkulturen GmbH (Braunschweig, Germany).

Mutant strains of UN4495 were in general constructed by cloning the respective genes by PCR-techniques, inserting a tetracycline resistance cassette derived from the MiniTn5 (DeLorenzo et al. 1990), cloning the respective interrupted genes into the suicide vector pKAS46 (Skorukpski and Taylor 1996) followed by transformation into the streptomycin resistant K. pneumoniae UN4495 strain (RAS46). Recombinant strains (generated by means of a double cross over) were identified by the ability to grow on LB supplemented with 400 $\mu \mathrm{g}$ streptomycin per $\mathrm{ml}$ and resistance to tetracycline (Skorukpski and Taylor 1996); the respective chromosomal mutations were confirmed by PCR and Southern blot analysis (Sambrock et al. 1989). For generating homologous primer for PCR amplification sequence information for genes of K. pneumoniae MG478578 (subsp. pneumoniae, not $\mathrm{N}_{2}$-fixing) were 
obtained from the database of the Genome Sequencing Center, Washington University, St. Louis (Genome Sequencing Center, personal communication) and using the database ERGO (Integrated Genomics, Inc.) (http://www.integratedgenomics.com).

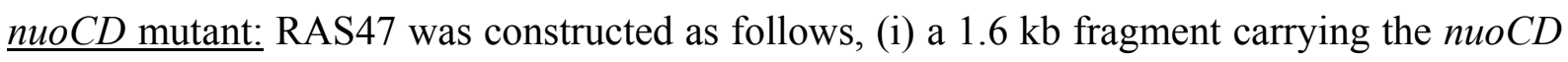
genes of $K$. pneumoniae M5a1 was amplified by PCR using primers with additional synthetic restriction recognition sites (underlined) nuoC/D ERI (5'CAGCGCGAATTCTCGCCGGCA3') and primer nuoC/D HindIII (5'CTGCTGAAGCTTGCGCAGACTCTG') and cloned into pBluescript $\mathrm{SK}^{+}$producing pRS191, (ii) a $2.2 \mathrm{~kb}$ fragment containing the tetracycline resistance cassette (DeLorenzo et al. 1990) was inserted into the EcoRV site of nuoCD gene region in pRS191 yielding pRS194, (iii) the $3.8 \mathrm{~kb} E c o \mathrm{RI} / K p n \mathrm{I}$ fragment of pRS191 carrying the interrupted nuoCD region was transferred into the allelic exchange vector pKAS46 (Skorukpski and Taylor 1996) creating plasmid pRS197; the correct insertion of the tetracycline cassette was checked by sequencing, (iv) pRS197 was transformed into RAS46 and recombinant strains carrying the chromosomally inserted plasmid by means of single homologous recombination were identified by their inability to grow on streptomycin agar plates as a consequence of the plasmid encoded rpsL mutation. Overnight selection of single colonies in liquid LB medium containing $400 \mu \mathrm{g}$ streptomycin per $\mathrm{ml}$ resulted in the loss of the integrated plasmid with an integration frequency of the interrupted nuoCD region in $50 \%$ of the integrands.

$f d n G$ mutant: Primer fdnG 5' EcoRI (5'CCGACTGATGAATTCCGACCGCGA3') and primer fdnG 3' HindIII (5'GCCGAGCAGAAGCTTGATCATCGC3') were used to clone a 1

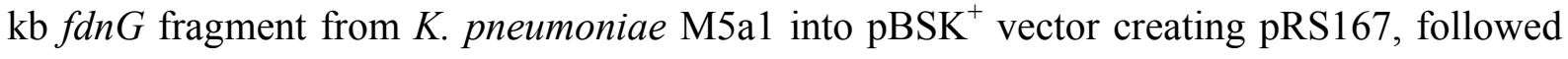
by insertion of the tetracycline resistance cassette into the EcoRV site of $f d n G$ fragment resulting in pRS177. The $3.2 \mathrm{~kb} E c o \mathrm{RI} / K p n \mathrm{I}$ fragment of pRS177 including the $f d n G:: t e t$ region was cloned into pKAS46. The construction of the K. pneumoniae chromosomal mutant was performed using the same strategy as described in detail above, yielding RAS48.

Growth conditions. E. coli and $K$ pneumoniae strains were grown anaerobically with molecular nitrogen $\left(\mathrm{N}_{2}\right)$ as gas phase at $30^{\circ} \mathrm{C}$ in minimal medium supplemented with $4 \mathrm{mM}$ glutamine as the sole nitrogen source (nitrogen limitation), $10 \mathrm{mM} \mathrm{Na}_{2} \mathrm{CO}_{3}, 0.3 \mathrm{mM}$ sulfide and $0.002 \%$ resazurin to monitor anaerobiosis (Schmitz et al. 1996). The medium was further supplemented with. $0.5 \%$ sucrose and $0.004 \%$ histidine for K. pneumoniae strains and 1\% glucose and $0.002 \%$ tryptophane for E. coli strains. Precultures were grown overnight in closed bottles with $\mathrm{N}_{2}$ as gas phase in the same medium but lacking sulfide and resazurin. 25 $\mathrm{ml}$ main cultures were inoculated from precultures and incubated under a nitrogen atmosphere 
and strictly anoxic conditions without shaking. Samples were taken anaerobically for monitoring the optical density at $600 \mathrm{~nm}$ and determining B-galactosidase activity. In E. coli strains carrying a plasmid encoding NifL and NifA (pNH3) or NifA alone (pJES851) expression of nifLA or nifA from the tac promoter was induced by the addition of $10 \mu \mathrm{M}$ IPTG (isopropyl-ß-D-thiogalactopyranoside).

B-Galactosidase assay. NifA-mediated activation of transcription from the nifHDK promoter in K. pneumoniae UN4495 and E. coli strains was monitored by measuring the differential rate of $\beta$-galactosidase synthesis during exponential growth (units per $\mathrm{ml}$ per optical density at $600 \mathrm{~nm}\left(\mathrm{OD}_{600}\right)$ (Schmitz et al. 1996)). Inhibitory effects of NifL on NifA activity were assessed by virtue of a decrease in nifH expression.

Purification of MBP-NifL. The fusion protein between maltose binding protein (MBP) and NifL was synthesized in NCM1529 carrying plasmid pJES794 (Narberhaus et al. 1995) growing aerobically at $30{ }^{\circ} \mathrm{C}$ in maximal induction medium (Mott et al. 1985) supplemented with $0.5 \mathrm{mM}$ riboflavin. Expression of the fusion protein was induced with $100 \mu \mathrm{M}$ IPTG when cultures reached an $\mathrm{OD}_{600}$ of 0.6. After harvesting and disruption in $\mathrm{B}$ buffer $(20 \mathrm{mM}$ Epps (N-[2-hydroxyethyl]piperazine- $N$ '-3-propanesulfonic acid), $125 \mathrm{mM}$ potassium glutamate, $5 \%$ glycerol, $1.5 \mathrm{mM}$ dithiothreitol, $\mathrm{pH}$ 8.0) using a French pressure cell, cells debris were sedimented by centrifugation at 20,000 x $g$ for $30 \mathrm{~min}$ and fusion proteins were purified from the supernatant by amylose affinity chromatography. All purification steps were performed at $4{ }^{\circ} \mathrm{C}$ in the dark preventing degradation of the FAD moiety. The purified protein was dialyzed overnight into B buffer containing $25 \mathrm{mM}$ potassium glutamate and subsequently used for biochemical analysis. The amount of FAD cofactor of the NifL fractions was calculated using a UV/Vis spectrum at $450 \mathrm{~nm}$ and the extinction coefficient $\epsilon_{450}=11.3 \mathrm{mM}^{-1} \mathrm{~cm}^{-1}$ (Whitby 1953). In general an FAD content of 0.4 to $0.6 \mathrm{~mol} \mathrm{FAD} \mathrm{/} \mathrm{mol}$ purified MBP-NifL was obtained.

Spectral analysis of purified MBP-NifL. Purified MBP-NifL was reduced under a $\mathrm{N}_{2}$ atmosphere in the presence of $\mathrm{NADH} / \mathrm{H}^{+}$and methyl viologen. The standard $0.2 \mathrm{ml}$ assay was performed in $\mathrm{B}$ buffer ( $25 \mathrm{mM}$ potassium glutamate, $\mathrm{pH}$ 8.0) under a nitrogen atmosphere using $40 \mu \mathrm{M}$ MBP-NifL. Reduction of fully oxidized MBP-NifL at room temperature was followed using a spectrophotometer with an integrated diode array detector (J\&M Analytische Meß- und Regeltechnik, Aalen, Germany). As reductants $1.25 \mathrm{mM} \mathrm{NADH} / \mathrm{H}^{+}$(final concentration) in the presence of $0.2 \mu \mathrm{M}$ methyl viologen or inverted vesicles $(10 \mathrm{mg} / \mathrm{ml})$ derived from $K$. pneumoniae cells and $0.12 \mathrm{mM}$ (final concentration) non-physiological 
electron donor, reduced dimethylnaphthoquinone $\left(\mathrm{DMN}_{\mathrm{red}}\right)$ was used in the absence of a redox mediator. Stock solution of DMN was prepared in methanol. After dilution into anaerobic B-buffer containing $25 \mathrm{mM}$ potassium glutamate, DMN was reduced by molecular hydrogen in the gas phase in the presence of platin oxide.

Preparation of inside-out vesicles of $\boldsymbol{K}$. pneumoniae. 11 cultures of $K$. pneumoniae cells were grown under nitrogen and oxygen-limited conditions, harvested at an optical density of $\mathrm{OD}_{600}=1.3$ and vesicles were prepared according to Krebs et al. (1999) except the addition of diisopropylfluorophosphate to the vesicle buffer. Inverted vesicles were directly used for the reduction of MBP-NifL or stored at $-70{ }^{\circ} \mathrm{C}$. All manipulations were performed under exclusion of oxygen in an anaerobic chamber at $4{ }^{\circ} \mathrm{C}$.

Determination of NADH:ubiquinone oxidoreductase activity. The enzyme activity of the NADH:ubiquinone oxidoreductase in cell extracts prepared under anaerobic conditions was determined as described by Friedrich et al. (1989) using ferricyanide as electron acceptor. The assay contained vesicle buffer (10 mM Tris/ $\mathrm{HCl} \mathrm{pH} 7.5,50 \mathrm{mM} \mathrm{KCl}, 2 \mathrm{mM}$ DTT), $0.3 \mathrm{mM}$ $\mathrm{NADH} / \mathrm{H}^{+}$and $0.2 \mathrm{mM}$ potassium ferricyanide. The reaction was started by adding cell extract and reduction of ferricyanide was monitored at $410 \mathrm{~nm}$.

Southern blot analysis. Southern blots were performed as described by Sambrock et al. (1989) using a vacuum pump for the DNA transfer. Hybridization with DIG-labeled probes and detection using CSPD as substrate was carried out according to the detection protocol of the manufacturer (Boehringer, Germany).

Western blot analysis. $1 \mathrm{ml}$ samples of exponentially growing cultures were harvested and concentrated 20-fold into sodium dodecyl sulfate (SDS) gel-loading buffer (Laemmli, 1970). Samples were separated by SDS/polyacrylamide (12\%) gel electrophoresis and transferred to nitrocellulose membranes as described (Sambrock et al. 1989). Membranes were exposed to polyclonal rabbit antisera directed against the NifL or NifA proteins of $K$. pneumoniae, protein bands were detected with secondary antibodies directed against rabbit immunoglobulin $\mathrm{G}$ and coupled to horseradish peroxidase (BioRad Laboratories). Purified NifA and NifL from K. pneumoniae and prestained protein markers (New England Biolabs, UK) were used as standards. 


\section{RESULTS}

Under oxygen and nitrogen limitation reduction of the flavin moiety of NifL results in a noninhibitory conformation of the NifL-protein. Localization analysis of $K$. pneumoniae NifL revealed that under those derepressed conditions NifL is membrane-associated, indicating that sequestration of NifL to the membrane is involved in the regulation of NifA activity by NifL. In order to analyze whether the association of NifL to the cytoplasmic membrane is accompanied with the reduction of NifL by a membrane-bound electron donor, we studied reduction of purified MBP-NifL in vitro and analyzed the influence of different oxidoreductases of the anaerobic electron transport chain on NifL reduction.

\section{K. pneumoniae NifL is reduced by $\mathrm{NADH} / \mathrm{H}^{+}$in the presence of a redox-mediator or} anaerobic inside-out vesicles. In order to demonstrate whether $\mathrm{NADH} / \mathrm{H}^{+}$is a potential electron donor in vivo, reduction of purified NifL was studied in vitro. In general, NifL was synthesized in maximal induction medium under aerobic conditions fused to the maltose binding protein (MBP) to keep NifL in a more soluble state. Subsequently MBP-NifL was purified to apparent homogeneity by affinity chromatography. The FAD content of those purified fractions was in the range of 0.4 - 0.6 FAD per MBP-NifL. Fully oxidized MBP-NifL $(40 \mu \mathrm{M})$ was incubated in an anaerobic cuvette under a nitrogen atmosphere in a total volume of $200 \mu \mathrm{l}$ B-buffer containing $25 \mathrm{mM}$ glutamate. The absorption spectra were recorded online using a diode array detector. In the absence of a redox mediator, the addition of $1.25 \mathrm{mM}$ $\mathrm{NADH} / \mathrm{H}^{+}$(final concentration) did not result in reduction of the NifL-bound FAD-cofactor even after long incubation periods up to $25 \mathrm{~min}$ (data not shown). However, in the presence of $0.2 \mu \mathrm{M}$ methyl viologen, significant reduction of the flavin-moiety of $\mathrm{NifL}$ by $\mathrm{NADH} / \mathrm{H}^{+}$was observed. After the addition of $\mathrm{NADH} / \mathrm{H}^{+}$the flavin-specific absorbance at $450 \mathrm{~nm}$ decreased constantly within 50 min indicating that the flavin cofactor of NifL was reduced by electrons derived from $\mathrm{NADH} / \mathrm{H}^{+}$(Fig. 15). This was further supported by the difference spectrum of oxidized MBP-NifL before the addition of $\mathrm{NADH} / \mathrm{H}^{+}$corrected versus the spectrum $50 \mathrm{~min}$ after $\mathrm{NADH} / \mathrm{H}^{+}$addition, which clearly showed the flavin-specific absorption maximum at $450 \mathrm{~nm}$ (inset of Fig. 15) and the $420 \mathrm{~nm}$ absorbance which is generally found in NifL preparations synthesized under nitrogen sufficiency (Klopprogge and Schmitz, 1999). These findings strongly indicate that $\mathrm{NADH} / \mathrm{H}^{+}$is a potential electron donor for NifL reduction in vivo, however it appears that the reducing equivalents derived from $\mathrm{NADH} / \mathrm{H}^{+}$have to be transferred to NifL through an additional oxidoreductase system. 


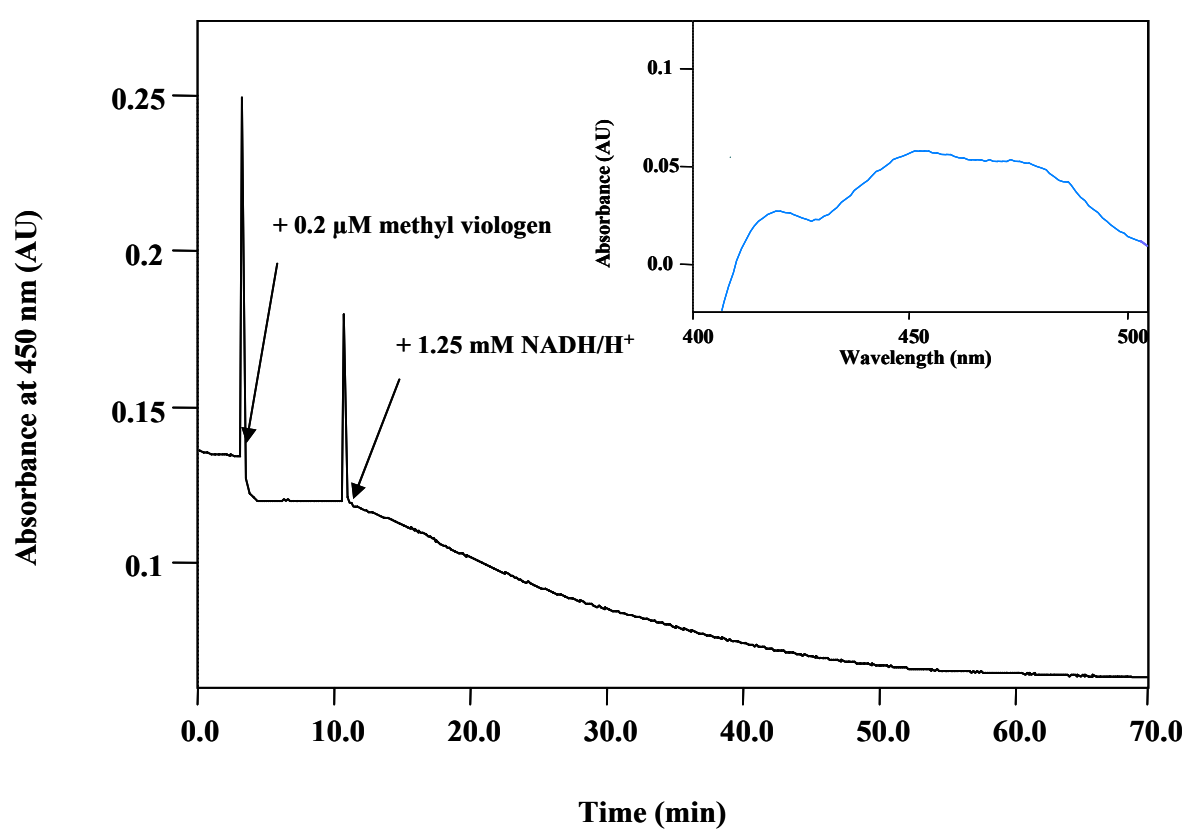

Fig. 15: Reduction of purified MBP-NifL with $\mathrm{NADH} / \mathrm{H}^{+}$in the presence of methyl viologen. $40 \mu \mathrm{M}$ purified fully oxidized MBP-NifL in B-buffer ( $\mathrm{pH}$ 8.0) was incubated in an anaerobic cuvette under a nitrogen atmosphere at $25^{\circ} \mathrm{C}$. After the addition of methyl viologen to a final concentration of $0.2 \mu \mathrm{M}$ the protein was reduced by the addition of $1.25 \mathrm{mM} \mathrm{NADH} / \mathrm{H}^{+}$(indicated by arrows). The spectral changes were recorded using a spectrophotometer with an integrated diode array detector (J \& M Analytische Mess- und Regeltechnik Aalen, Germany) and the reduction of the flavin moiety of the protein was monitored at $450 \mathrm{~nm}$. The inset shows the difference spectrum; the fully oxidized spectrum at $10 \mathrm{~min}$ was corrected versus the reduced spectrum at $60 \mathrm{~min}$.

To obtain further evidence for NifL reduction by $\mathrm{NADH} / \mathrm{H}^{+}$via an oxidoreductase system in vivo we analyzed the effect of inside-out vesicles derived from K. pneumoniae cells on the reduction state of NifL. As NifL is membrane-associated under oxygen- and nitrogenlimitation oxidoreductases of the anaerobic electron transport chain are attractive candidates for transferring electrons derived from $\mathrm{NADH} / \mathrm{H}^{+}$to membrane-bound NifL. To exclude the presence of contaminating redox mediators in the following experiments the cuvettes were extensively washed with chromosulfuric acid and control experiments were performed, in which no significant decrease of the NifL absorbance at $450 \mathrm{~nm}$ was observed after the addition of $\mathrm{NADH} / \mathrm{H}^{+}$. Inside-out vesicles were prepared under strictly anaerobic conditions from $K$. pneumoniae cells grown under nitrogen and oxygen limitation to obtain vesicles containing the anaerobic electron transport chain (see Materials and Methods). Fully oxidized MBP-NifL was incubated under a nitrogen atmosphere and the absorption spectrum was 
recorded online. The addition of $100 \mu \mathrm{g}$ inside-out vesicles resulted in a slow decrease of flavin specific absorbance at $450 \mathrm{~nm}$, suggesting that NifL is partially reduced by electrons derived from reduced membrane-bound oxidoreductases (Fig. 16 A). This reduction process of NifL-bound FAD was further increased by the addition of $\mathrm{NADH} / \mathrm{H}^{+}$. Again, the difference spectrum of NifL before $\mathrm{NADH} / \mathrm{H}^{+}$addition corrected versus the spectrum $32 \mathrm{~min}$ after the addition of $\mathrm{NADH} / \mathrm{H}^{+}$clearly showed a significant decrease of the flavin-specific absorbance at $450 \mathrm{~nm}$ (Fig. $16 \mathrm{~B}$ ). The finding, that NifL was reduced by a membrane-bound oxidoreductase system receiving reducing equivalents derived from $\mathrm{NADH} / \mathrm{H}^{+}$strongly indicates that in vivo the NifL-bound FAD cofactor receives electrons from a reduced membrane-bound oxidoreductase system.

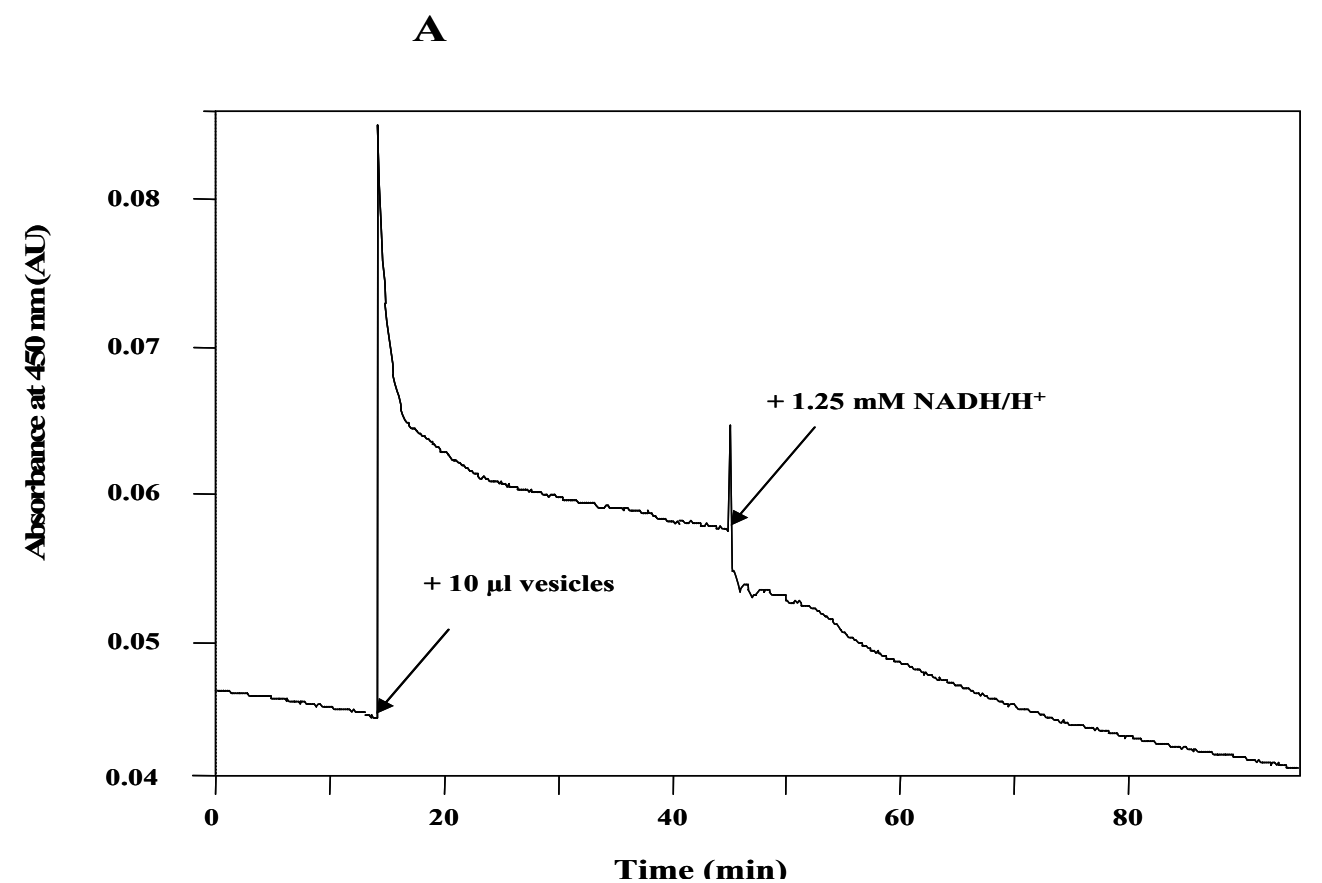

Fig. 16: Reduction of purified MBP-NifL with $\mathrm{NADH} / \mathrm{H}^{+}$in the presence of inverted vesicles from $K$. pneumoniae. $40 \mu \mathrm{M}$ purified fully oxidized MBP-NifL was incubated in an anaerobic cuvette under a nitrogen atmosphere at $25^{\circ} \mathrm{C}$ in a final volume of $400 \mu \mathrm{B}$ buffer. $30 \mathrm{~min}$ after the addition of $10 \mu 1$ inverted vesicles (10 $\mathrm{mg} / \mathrm{ml}$ ) of K. pneumoniae cells grown under nitrogen and oxygen limitation, the reduction was started by the addition of $1.25 \mathrm{mM} \mathrm{NADH} / \mathrm{H}^{+}$(final concentration). Changes in absorbance upon the reduction of the flavin cofactor were recorded and monitored as described in the legend of figure 15. (A) Time course measurement at $450 \mathrm{~nm}$ of the MBP-NifL reduction with $\mathrm{NADH} / \mathrm{H}^{+}$in the presence of inverted vesicles. (B) Absorbance spectra of MBP-NifL after vesicle injection at 40 min (oxidized MBP-NifL) and after $\mathrm{NADH} / \mathrm{H}^{+}$addition at $85 \mathrm{~min}$ (reduced MBP-NifL). The inset shows the corresponding difference spectrum of oxidized MBP-NifL corrected versus the reduced spectrum. 


\section{B}

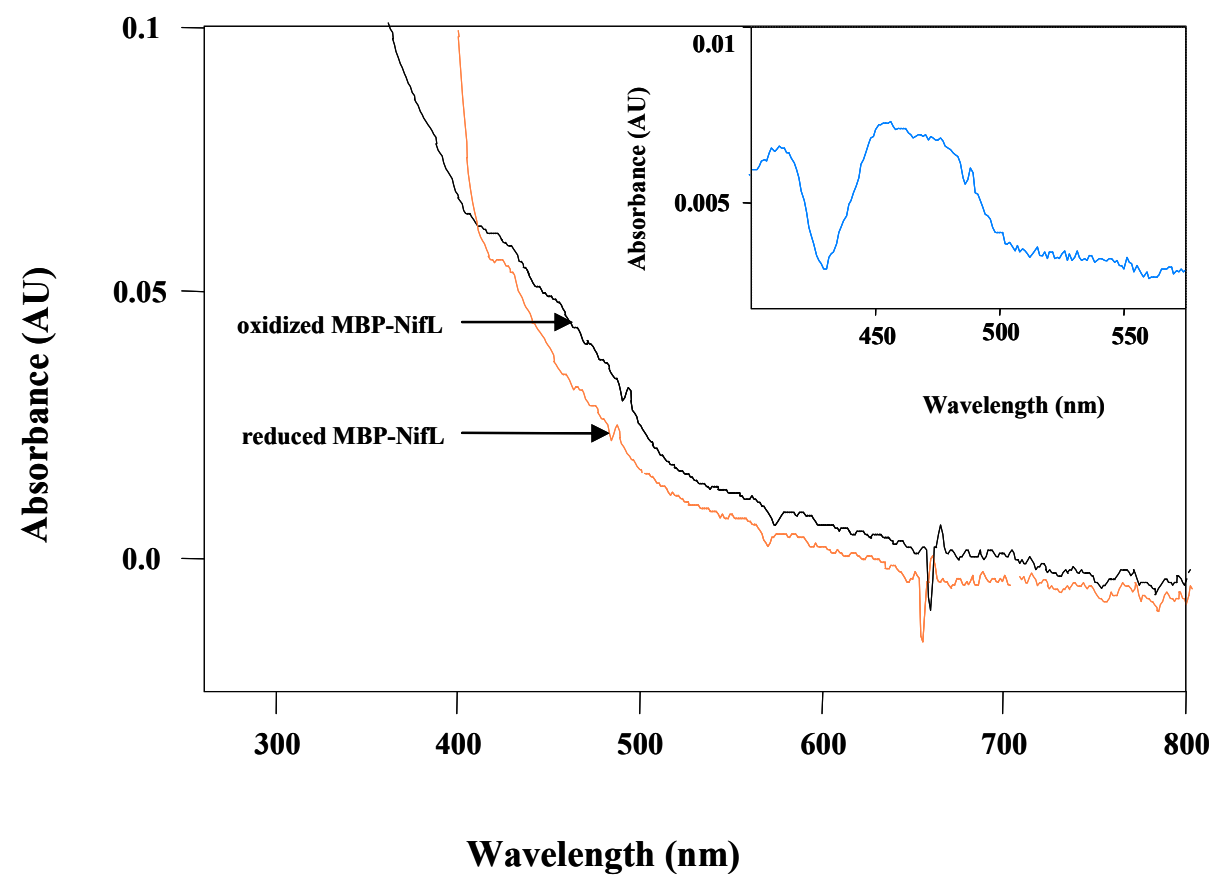

Effects of chromosomal $\boldsymbol{n d h}$ and frd null mutations on $n$ if induction in a heterologous $E$. coli system. The biochemical analyses of purified MBP-NifL and membrane association of NifL under derepressing conditions indicate that during the process of nif regulation the NifLbound cofactor is apparently reduced by a membrane-bound oxidoreductase system. To obtain further evidence we studied the influence of two oxidoreductase systems on nif regulation in a heterologous E. coli system: (i) NADH dehydrogenaseII and (ii) fumarate reductase, which are both under transcriptional control of Fnr.

NCM1529 carrying a chromosomal nif $H^{\prime}-{ }^{\prime} l a c Z$ fusion was used as parental strain. The $K$. pneumoniae regulatory proteins NifL and NifA were synthesized from plasmids, pNH3 (nifLA) or pJES851 (nifA) at induction levels, at which NifL function in E. coli is regulated normally in response to oxygen and combined nitrogen (He et al. 1997, Grabbe et al. 2001). To study the effect of the two oxidoreductases the respective null alleles, ndh::tet (NADH dehydrogenaseII) and frd::tet (fumarate reductase), were introduced by $\mathrm{P} 1$ transduction into the parental strain NCM1529 as described in Materials and Methods. After introducing nifLA and nifA on plasmids, the resulting strains were grown anaerobically in minimal medium with glucose as carbon source and glutamine as sole nitrogen source to study the effects of the 
mutation on NifA regulation by NifL. No significant differences in growth rates or in the NifL and NifA expression levels were obtained for the mutant and the respective parental strains (Table 8). Transcription of nifH'- lacZ fusion dependent on NifA activity was monitored in the two mutant backgrounds by determining synthesis rates of ß-galactosidase during exponential growth (Schmitz et al. 1996). In case of the frd mutation no effects on nif induction was detectable. Synthesis rates of $\beta$-galactosidase determined for the frd mutant strain (RAS54) showed no significant difference compared to the parental strain (NCM1528) (Table 8). However, in the absence of a functional NADH dehydrogenaseII (RAS51), expression of $n i f H^{\prime}$ significantly decreased resulting in a $\beta$-galactosidase synthesis rate of 300 $\pm 20 \mathrm{U} / \mathrm{ml} / \mathrm{OD}_{600}$, which is equivalent to $10 \%$ of the synthesis rate in the parental strain NCM1528 (3000 $\left.\pm 100 \mathrm{U} / \mathrm{ml} / \mathrm{OD}_{600}\right)$. In contrast, the $n d h$ mutant strain carrying NifA alone on a plasmid (RAS52) showed no significant decrease in NifA activity compared to the parental strain (NCM1527), indicating that NifA activity is not directly affected by the $n d h$ mutation. These findings suggest that in the absence of NADH dehydrogenaseII NifL apparently does not receive the signal for anaerobiosis and inhibits NifA activity. Thus, in the heterologous E. coli system NADH dehydrogenaseII may be responsible for reduction of membrane-associated NifL under anaerobic conditions.

Table 8: Effects of chromosomal $n d h$ and $f r d$ null mutations on NifA activity in the heterologous $E$. coli system carrying $K$. pneumoniae nifL $A$ on a plasmid. E. coli strains carrying a single copy of a $\phi\left(n i f^{\prime \prime-}\right.$ lacZ) fusion (He et al. 1998) and K. pneumoniae nifLA (pNH3) or nifA (pJES851) under the control of the tac promoter were grown at $30^{\circ} \mathrm{C}$ in minimal medium under anaerobic conditions with $4 \mathrm{mM}$ glutamine as limiting nitrogen source. Expression of NifL and NifA was induced with $10 \mu \mathrm{M}$ IPTG. Synthesis rates of $ß$-galactosidase were determined reflecting NifA activity in the respective strain background.

\begin{tabular}{|c|c|c|c|}
\hline Strain & Relevant genotype & $\begin{array}{c}\text { Expression of } \text { nifH'-lacZ }^{\prime} \\
\left(\mathrm{U} / \mathrm{min} / \mathrm{OD}_{600}\right)^{\mathrm{a}}\end{array}$ & $\begin{array}{c}\text { Doubling time } \\
(\mathrm{h})\end{array}$ \\
\hline NCM1528 & Wild type/Ptac-nifLA & $3000 \pm 100$ & 5.0 \\
\hline NCM1527 & Wild type/Ptac-nifA & $5000 \pm 200$ & 4.8 \\
\hline RAS51 & ndh/Ptac-nifLA & $300 \pm 20$ & 5.5 \\
\hline RAS52 $^{\mathrm{b}}$ & ndh/Ptac-nifA & $4500 \pm 150$ & 5.2 \\
\hline RAS54 $^{\mathrm{c}}$ & frd/Ptac-nifLA & $3500 \pm 100$ & 5.0 \\
\hline RAS55 & frd/Ptac-nifA & $5400 \pm 150$ & 4.9 \\
\hline
\end{tabular}

${ }^{\text {a }}$ Data presented as mean values ( \pm standard aberration) of at least three independent experiments.

${ }^{\mathrm{b}}$ Strain contains the $n d h:: t e t$ allele from ANN001 (T. Friedrich, unpublished results).

${ }^{c}$ Strain contains the frdABCD::tet allele from JI222 (Imlay, 1995) 


\section{NADH:ubiquinone oxidoreductase and formate dehydrogenaseN are effecting nif} regulation in $K$. pneumoniae. As the findings using the heterologous E. coli system indicated that NADH dehydrogenaseII might be the physiological electron donor for NifL reduction, we intended to construct a chromosomal null allele of the respective homologous gene in $K$. pneumoniae. In order to clone the $n d h$ gene by PCR techniques we generated primers based on the $5^{\prime}$ and $3^{\prime}$ end sequences of the $n d h$ gene of $K$. pneumoniae MGH78578 (subsp. pneumoniae); sequence information was obtained from the database from the Genome Sequencing Center, Washington University St. Louis (Genome Sequencing Center, personal communication). Using chromosomal DNA from $K$. pneumoniae subsp. pneumoniae (DSM No. 4799) as control, the $n d h$ gene was amplified. However, no corresponding PCR product was obtained using chromosomal DNA from K. pneumoniae M5a1, although different pairs of homologous and degenerated primers were tested under various PCR-assay conditions. To confirm this finding we performed Southern blot analysis of SmaI digested chromosomal DNA derived from K. pneumoniae M5a1, K. oxytoca (DSM No. 4798) and K. pneumoniae subsp. pneumoniae using a DIG-labeled $n d h$ probe derived from $K$. pneumoniae subsp. pneumoniae. Hybridization resulted in a single hybridization signal (4 kbp) for $K$. pneumoniae subsp. pneumoniae DNA (Fig. 17), which is in accordance with the expected fragment size based on the knowledge of the genomic sequence. However, no signal was obtained for DNA derived from K. pneumoniae M5a1 and $K$. oxytoca even under conditions of low stringency. These findings strongly indicate that the nitrogen fixing strain $K$. pneumoniae M5a1 and $K$. oxytoca do not exhibit an NADH-dehydrogenaseII. Thus, we decided to examine the influence of two other membrane-bound oxidoreductases of the anaerobic respiration on nif regulation in K. pneumoniae. 


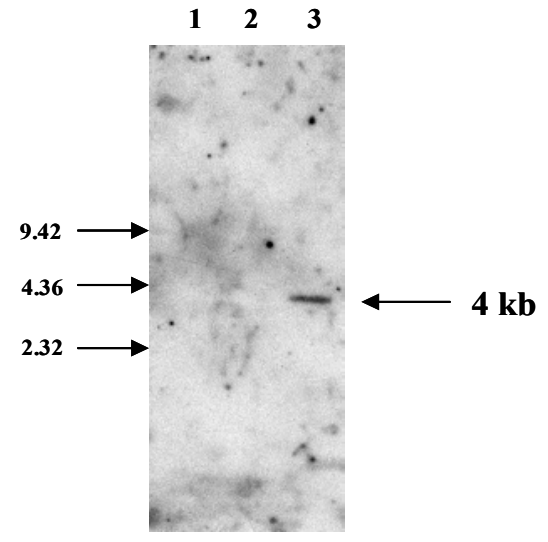

Fig. 17: Southern blot analysis using a ndhII probe derived from $K$. pneumoniae subs. pneumoniae. Genomic DNA from K. pneumoniae strains was completely digested with SmaI and equal amounts analyzed by Southern hybridization performed according to Sambrock et al. (1989) using a DIG-labeled ndhII probe (see Materials and methods). Lane 1, K. pneumoniae M5a1; lane 2, K. oxytoca (DSM 4798); lane 3, K. pneumoniae subs. pneumoniae (DSM 4799). Numbers on the left are molecular sizes in kilobases, the estimated size of the hybridizing fragment is indicated on the right.

K. pneumoniae strain UN4495 carrying nifLA and a nifK'- lacZ fusion on the chromosome was used as parental strain which allows to monitor NifA mediated transcription from the nifHDK promoter by measuring the differential rates of $\beta$-galactosidase synthesis during exponential growth (Schmitz et al. 1996). Two mutant strains of K. pneumoniae UN4495 were constructed carrying either a chromosomal nuoCD null allele (encoding for subunits $\mathrm{C}$ and D of the coupling NADH: ubiquinone oxidoreductase) or a chromosomal $f d n G$ null allele (encoding for the $\gamma$ subunit of formate dehydrogenaseN). The mutant strains were constructed by cloning the respective genes, inserting a tetracycline resistance cassette derived from the MiniTn5 (DeLorenzo et al. 1990) and introducing the interrupted genes into the $K$. pneumoniae UN4495 chromosome using the allelic exchange system described by Skorupsky and Taylor (1996) (see Materials and Methods). The disruption of $n u o C D$ and $f d n G$ in the respective mutant strains was confirmed by PCR and Southern blot analysis (data not shown). In addition, the nuoCD mutant strain (RAS47) was further characterized biochemically by determining the specific activity of NADH-oxidation in anaerobic cell extracts. The specific activity of NADH-oxidation obtained for the nuoCD mutant strain $(0.4 \mathrm{U} / \mathrm{mg}$ cell extract 
protein) was significantly lower than the oxidation rate determined for the parental strain (10 $\mathrm{U} / \mathrm{mg}$ cell extract protein) and might be based on unspecific NADH-oxidation (Fig. 18). In contrast, the residual NADH-oxidation rate of an E. coli nuo mutant strain was determined to be equivalent to $20 \%$ of the $\mathrm{NADH}$-oxidation rate obtained for the parental strain and is supposed to be dependent on NADH-dehydrogenaseII activity (Falk-Krzesinski and Wolfe, 1998). Thus, the significantly lower residual NADH-oxidation rate of the K. pneumoniae nuo mutant strain (4\%) in comparison to the respective $E$. coli mutant further supports our finding that K. pneumoniae M5a1 does not exhibit a second NADH-dehydrogenase.

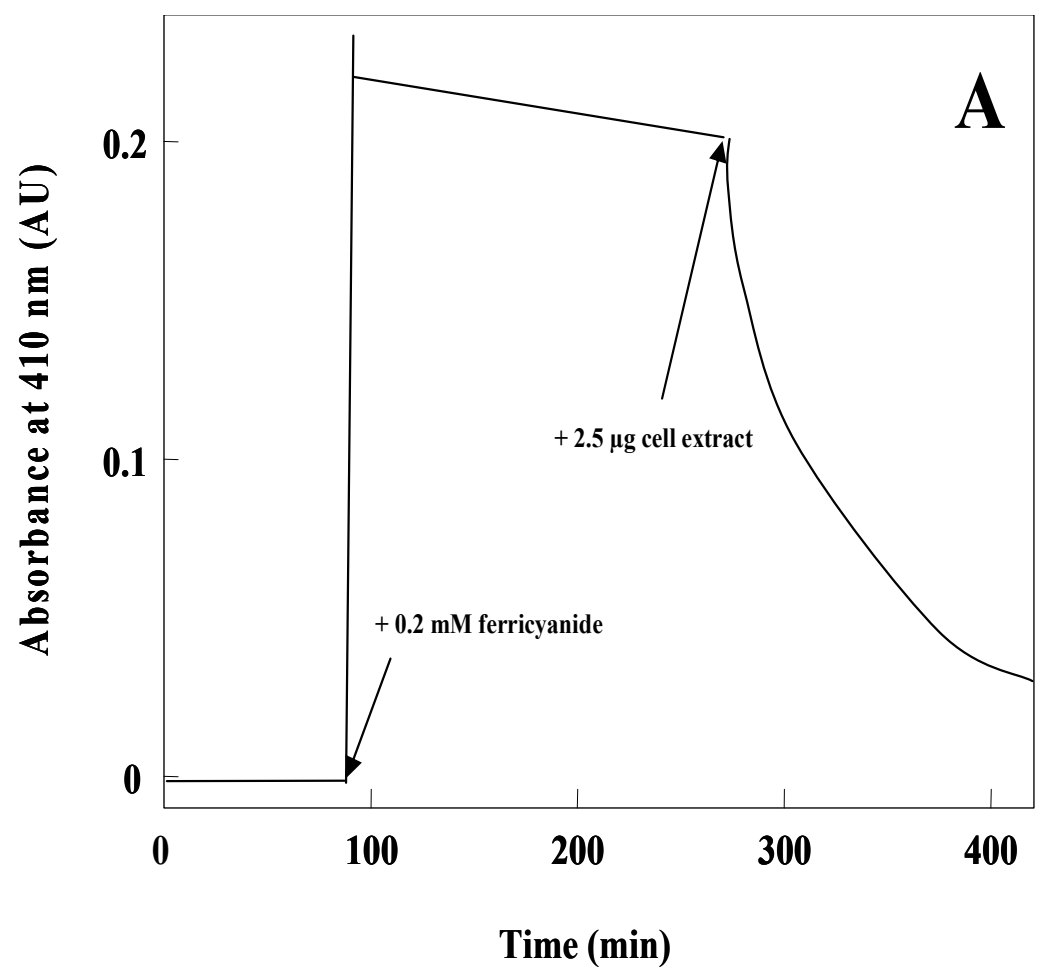




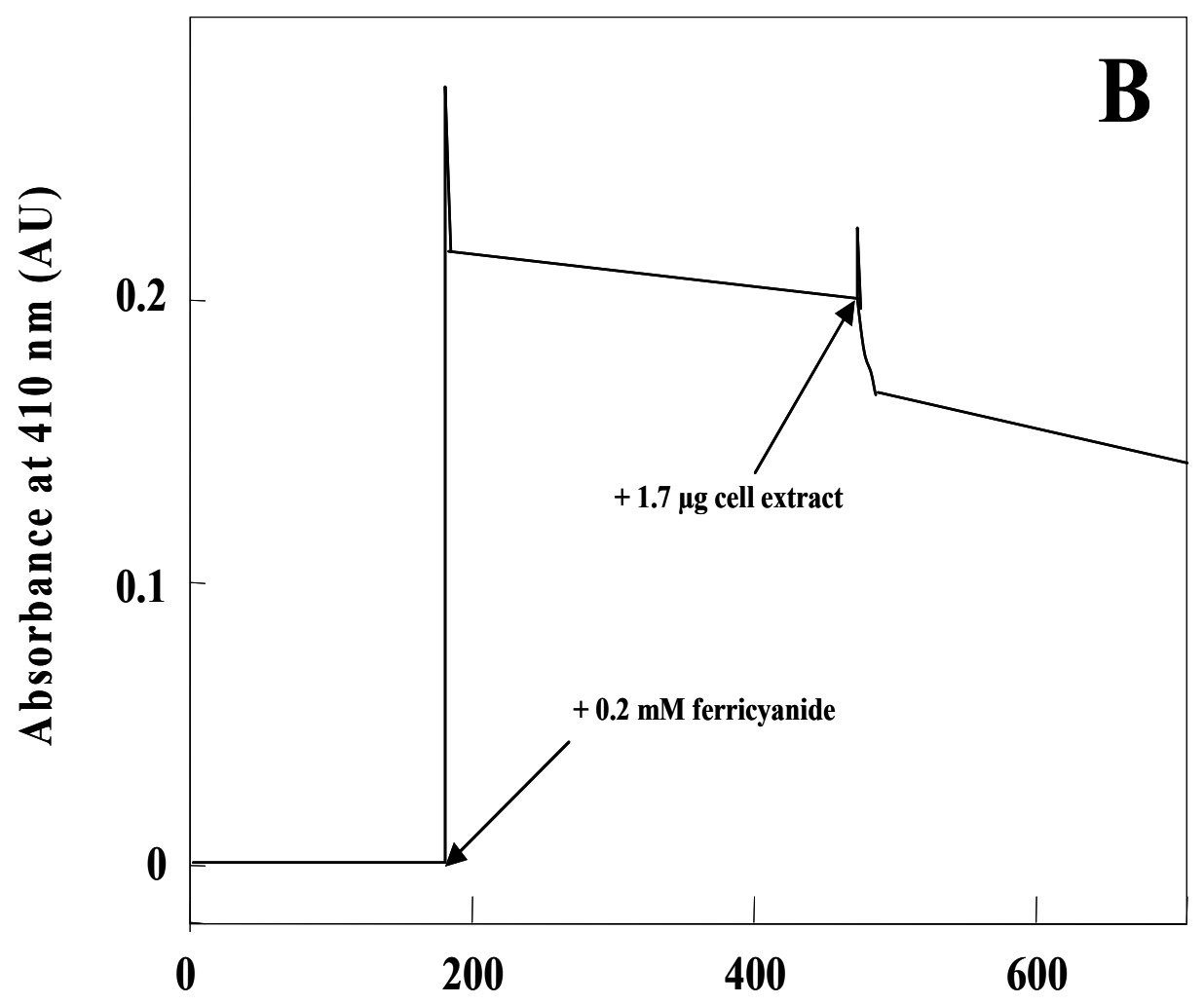

\section{Time(sec)}

Fig. 18: Determination of NADH:ubiquinone oxidoreductase activity in $\boldsymbol{K}$. pneumoniae. Activity of NADH oxidation by NADH:ubiquinone oxidoreductase was determined in cell extracts of anaerobically grown cultures (see Materials and methods). The decrease of absorption reflecting reduction of ferricyanide by NADH/ $\mathrm{H}^{+} \mathrm{was}$ monitored at $410 \mathrm{~nm}$. (A) wild type (UN4495) and (B) nuoCD mutant strain (RAS47). The addition of ferricyanide and crude cell extract is indicated by arrows.

$K$. pneumoniae wild type and the respective mutant strains were grown in minimal medium under oxygen limitation with glutamine as sole nitrogen source to exclude NifA inhibition by NifL in response to ammonium. Both mutant strains showed increased doubling times $(\mathrm{td}=5$ h) compared to the parental strain UN4495 $(\mathrm{td}=3.5 \mathrm{~h})$. This decrease in growth rates under anoxic conditions indicates that in the absence of either NADH:ubiquinone oxidoreductase or formate dehydrogenaseN the energy yield per mol glucose decreased based on reduced anaerobic respiration and increased fermentative recycling of $\mathrm{NAD}^{+}$from $\mathrm{NADH} / \mathrm{H}^{+}$. Unexpected both, the $n u o C D$ and the $f d n G$ mutant strain, showed significantly reduced levels of $\beta$-galactosidase synthesis rates under derepressing conditions (Fig. 19). The $\beta$-galactosidase synthesis rates determined for the $f d n G$ mutant strain RAS48 $\left(400 \pm 30 \mathrm{U} / \mathrm{ml} / \mathrm{OD}_{600}\right)$ were 
similar to synthesis rates found in the fnr mutant RAS18 (Fig. 19 B, Grabbe et al. 2001b). The observed nif induction level determined for the $K$. pneumoniae nuo mutant strain decreased even more dramatically to levels of approximately $60 \mathrm{U} / \mathrm{ml}^{-} \mathrm{OD}_{600}$ (Figure $19 \mathrm{~B}$ ), which is in the range of nif induction in the presence of oxygen and indicates that all NifL protein is in the oxidized inhibitory conformation (Grabbe et al. 2001b). Determination of NifL and NifA proteins in the mutant strain revealed no differences in the amount of the regulatory proteins compared to those of the parental strain (data not shown). These findings clearly show that in $K$. pneumoniae apparently more than one membrane-bound oxidoreductase system can provide electrons for NifL reduction under anaerobic conditions. They further indicate that NADH:ubiquinone oxidoreductase appears to play a major role in providing reducing equivalents for NifL in vivo.

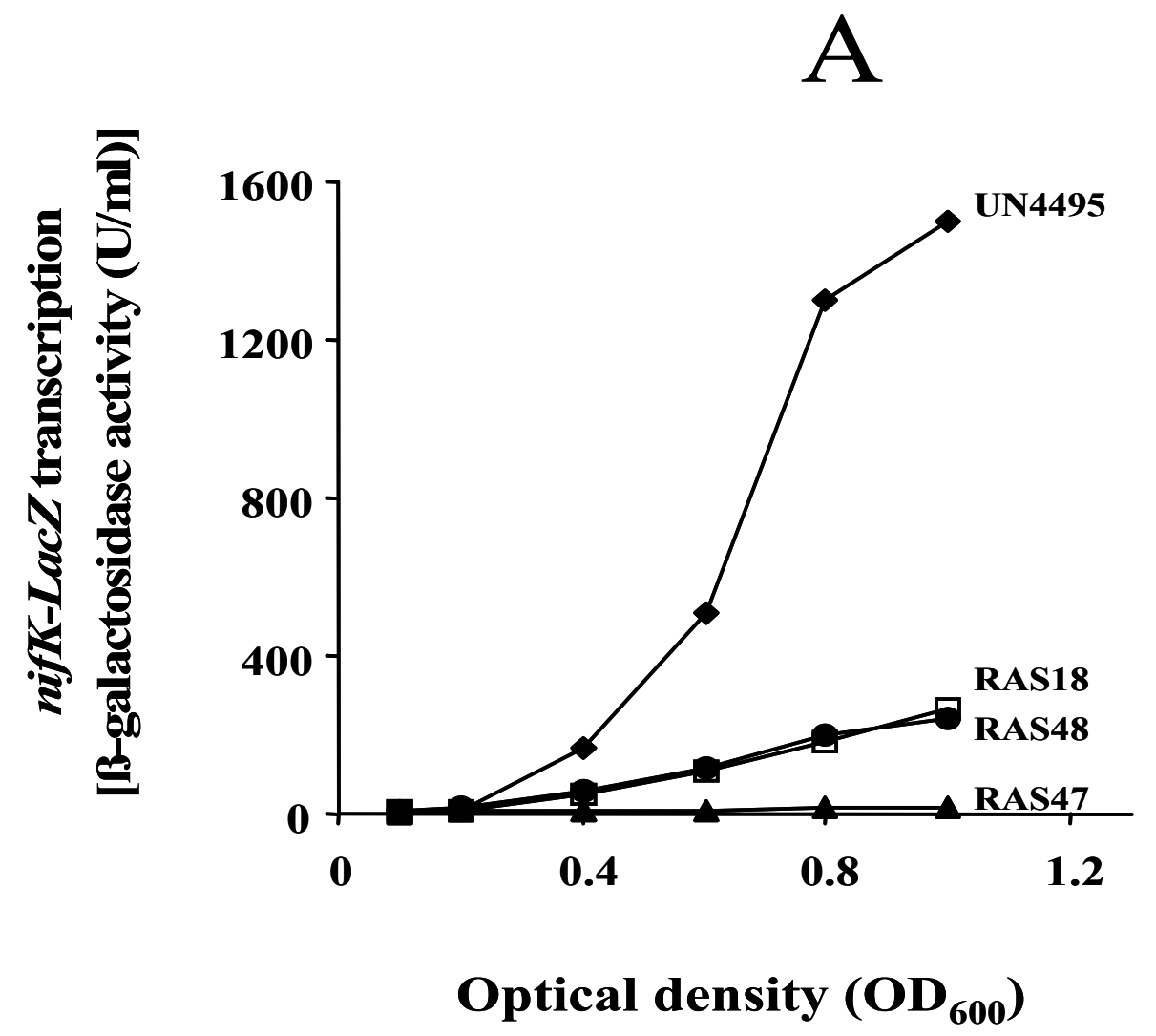




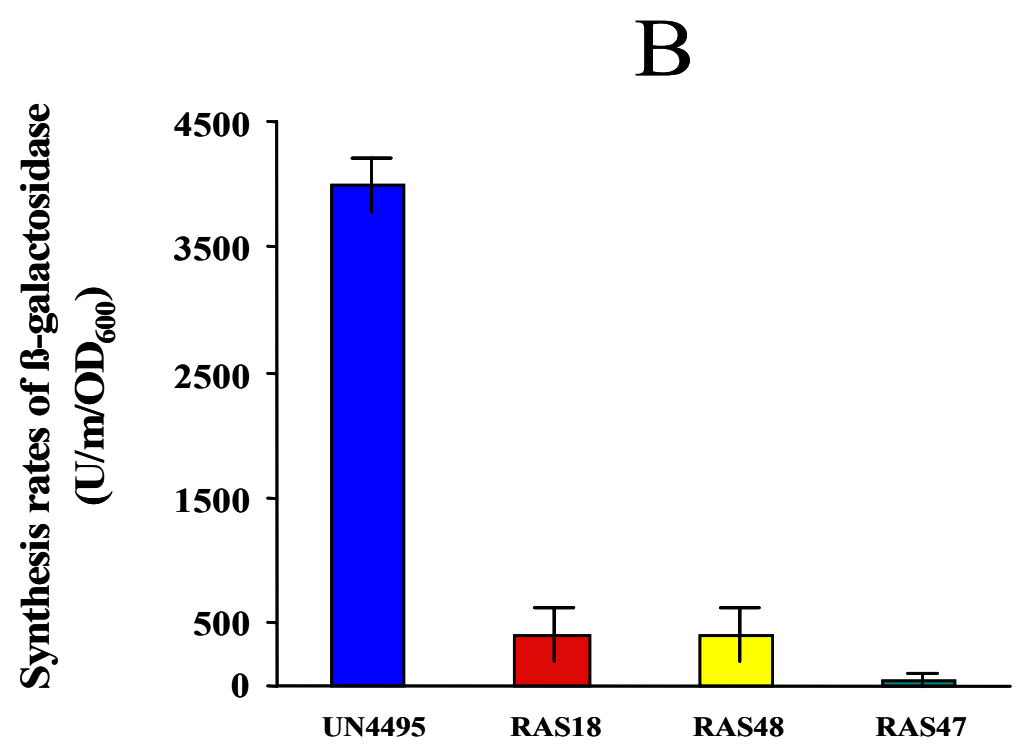

Strains

Fig. 19: Effects of chromosomal deletions in gene clusters encoding NADH:ubiquinone oxidoreductase (nuo) and formate dehydrogenaseN ( $f d n)$ on NifA activity in $K$. pneumoniae UN4495. NifA-mediated activation of transcription from the nifHDK-promoter in K. pneumoniae UN4495 and mutant derivatives was monitored by measuring the $\beta$-galactosidase activity during anaerobic growth at $30^{\circ} \mathrm{C}$ in minimal medium with glutamine $(4 \mathrm{mM})$ as limiting nitrogen source. Activities of $\beta$-galactosidase were plotted as a function of $\mathrm{OD}_{600}$ for K. pneumoniae UN4495 (wild type), the fnr mutant strain of UN4495 (RAS18), the $f d n G$ mutant strain of UN4495 (RAS48) and the nuoCD mutant strain of UN4495 (RAS47) carrying a chromosomal nifK'-'lacZ fusion (A). Synthesis rates of $\beta$-galactosidase from the nifHDK promoter were determined from the slope of these plots and are presented as bars reflecting nif-induction in the respective K. pneumoniae strains (B).

Effects of additional electron acceptors on nif regulation in $K$. pneumoniae. The finding that more than one oxidoreductase system can provide electrons for NifL reduction in vivo indicates that NifL apparently receives electrons at the cytoplasmic membrane provided by the quinone pool. To obtain additional evidence we studied nif induction in $K$. pneumoniae strain UN4495 in the presence of additional electron acceptors. The cultures were grown under nitrogen- and oxygen-limitation with glutamine as sole nitrogen source and sucrose, glucose or glycerol as carbon and energy source. In general, nif induction was significantly reduced when growing with glycerol as carbon and energy source and was equivalent to $25 \%$ of the induction level obtained with sucrose (Table 9). As we assayed nif induction by determining the differential rate of $\beta$-galactosidase synthesis, the calculated induction levels 
are normalized for differences in growth rates. Thus, the observed reduction of nif induction when growing on glycerol appears to be based on the lower energy charge of glycerol grown cells compared to cells grown with glucose. Supplementing the medium with the additional electron acceptors nitrate or fumarate did not effect nif induction when cells were growing on sucrose or glucose (Table 9). This indicates that the presence of nitrate, which is also an alternative nitrogen source, does not repress nif induction. However, when growing on glycerol, the presence of nitrate resulted in a significant decrease of nif induction $(200 \pm 20$ $\left.\mathrm{U} / \mathrm{ml} / \mathrm{OD}_{600}\right)$ compared to cells grown on glycerol in the absence of nitrate $(1000 \pm 20$ $\mathrm{U} / \mathrm{ml} / \mathrm{OD}_{600}$ ). No effect was observed when fumarate was added (Table 9). The growth rate did not change significantly upon the addition of fumarate or nitrate as it is also reported for E. coli (Tran and Unden, 1998). Taking together, these findings indicate that at conditions of low cellular energy charge, e.g. anaerobic growth on glycerol, electrons from the quinone pool are preferentially transferred onto nitrate via respiratory nitrate reductase to obtain higher energy yields. Thus, fewer electrons from the quinone pool are available to reduce NifL, resulting in the inhibition of NifA activity. Fumarate apparently is not competing for electrons as no effect on nif induction is observed in the presence of fumarate.

Table 9: Effects of additional electron acceptors on the nif induction in $K$. pneumoniae using different carbon and energy sources. $K$. pneumoniae UN4495 cultures were grown anaerobically at $30{ }^{\circ} \mathrm{C}$ in minimal medium with $4 \mathrm{mM}$ glutamine as limiting nitrogen source. The medium contained sucrose $(0.5 \%)$, glucose $(0.8 \%)$ or glycerol ( $1 \%)$ as carbon and energy source; fumarate or nitrate were added to a final concentration of $20 \mathrm{mM}$. Differential rates of transcription from the nifHDK-promoter were determined, reflecting the nifinduction under the respective growth conditions.

\begin{tabular}{|c|c|c|c|}
\hline $\begin{array}{c}\text { Carbon and } \\
\text { energy source }\end{array}$ & $\begin{array}{c}\text { Additional electron } \\
\text { acceptor }\end{array}$ & $\begin{array}{c}\text { B-galactosidase activity } \\
\left(\mathrm{U} / \mathrm{ml}^{\mathrm{O}} / \mathrm{OD}_{600}\right)^{\mathrm{a}}\end{array}$ & $\begin{array}{c}\text { Doubling time } \\
(\mathrm{h})\end{array}$ \\
\hline sucrose & - & $4000 \pm 100$ & 3.5 \\
\hline sucrose & fumarate & $4100 \pm 150$ & 3.5 \\
\hline sucrose & nitrate & $3900 \pm 150$ & 3.5 \\
\hline glucose & - & $3000 \pm 90$ & 3.5 \\
\hline glucose & fumarate & $2850 \pm 85$ & 3.5 \\
\hline glucose & nitrate & $3100 \pm 90$ & 3.5 \\
\hline glycerol & - & $1000 \pm 40$ & 5.5 \\
\hline glycerol & fumarate & $1100 \pm 60$ & 5.7 \\
\hline glycerol & nitrate & $200 \pm 20$ & 5.5 \\
\hline
\end{tabular}

${ }^{a}$ Data are presented as mean values ( \pm standard aberration) of 3 independent experiments. 
Reduced dimethylnaphthoquinone is able to reduce the flavin cofactor of MBP-NifL. In order to verify our finding that under derepressing conditions NifL receives electrons from the quinone pool of the anaerobic electron transport chain we examined whether reduced quinone derivatives can transfer electrons onto NifL. Dimethylnaphthoquinone (DMN) was reduced with molecular hydrogen in the presence of platin oxide and the reduction was confirmed by monitoring the changes in absorbance at 270 and $290 \mathrm{~nm}$. Fully oxidized MBP-NifL was incubated in an anaerobic cuvette under a nitrogen atmosphere in the absence of a redox mediator in a total volume of $200 \mu \mathrm{l}$ B-buffer and the absorption spectrum was recorded online using a diode array detector. After the addition of $120 \mu \mathrm{M} \mathrm{DMN}_{\text {red }}$ the flavin-specific absorbance at $450 \mathrm{~nm}$ decreased significantly indicating that electrons are transferred from $\mathrm{DMN}_{\text {red }}$ to the FAD-cofactor of NifL (Fig. 20). The reduction of NifL-bound FAD by $\mathrm{DMN}_{\text {red }}$ was confirmed by analyzing the difference spectrum of oxidized MBP-NifL (before $\mathrm{DMN}_{\text {red }}$ addition) corrected versus the spectrum $110 \mathrm{~min}$ after the addition of $\mathrm{DMN}_{\text {red, }}$, which showed the flavin-specific absorption maxima at 450 and $350 \mathrm{~nm}$ (inset Fig. 20). The finding that $\mathrm{DMN}_{\text {red }}\left(\mathrm{E}^{\circ}=-240 \mathrm{mV}\right)$ transfers electrons onto NifL-bound FAD strongly supports the model that in vivo NifL is reduced at the cytoplasmic membrane and receives electrons from the anaerobic quinone pool.

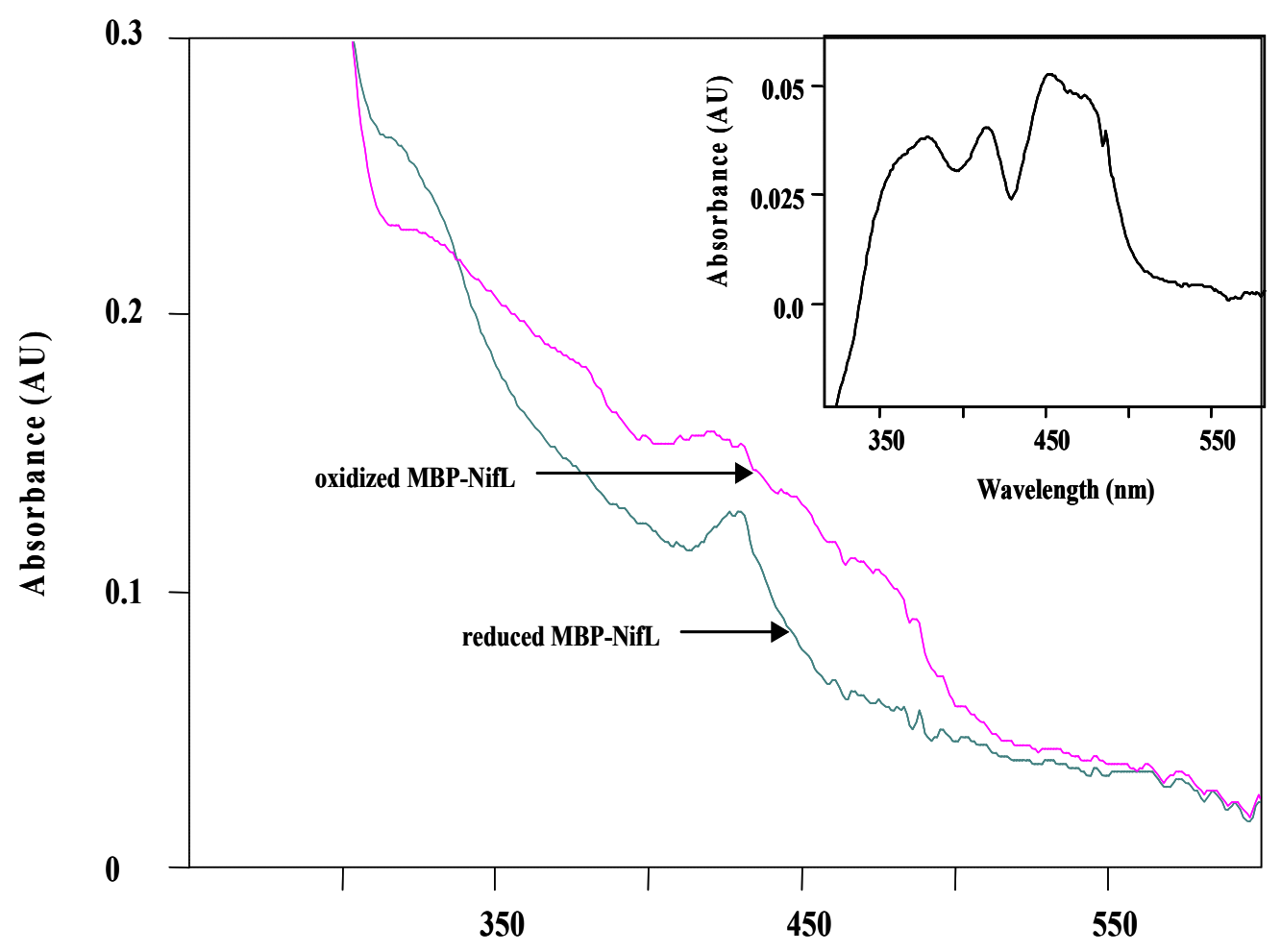

Wavelength (nm) 
Fig. 20: Reduction of MBP-NifL using reduced dimethylnaphthoquinone as artificial electron donor. 40 $\mu \mathrm{M}$ fully oxidized MBP-NifL was incubated in B buffer under a nitrogen atmosphere at room temperature. Reduced dimethylnaphthoquinone $\left(\mathrm{DMN}_{\text {red }}\right)$ was added to a final concentration of $0.2 \mathrm{mM}$ and the changes in absorbance were recorded using a spectrophotometer with an integrated diode array detector (J \& M Analytische Mess- und Regeltechnik Aalen, Germany). Absorbance spectra of MBP-NifL before (oxidized MBP-NifL) and 40 min after the addition of $0.2 \mathrm{mM} \mathrm{DMN}_{\text {red }}$ (reduced MBP-NifL) are shown. The corresponding difference spectrum of oxidized MBP-NifL corrected versus the reduced spectrum after addition of $\mathrm{DMN}_{\text {red }}$ is visualized in the inset.

\section{DISCUSSION}

The NifL-bound FAD receives electrons from the reduced quinone pool at the cytoplasmic membrane. We recently showed that in K. pneumoniae membrane-sequestration of NifL under nitrogen- and oxygen-limited conditions seems to be the mechanism for nif regulation in response to molecular oxygen and combined nitrogen (Klopprogge et al. 2002). In order to verify our model that membrane-association of NifL is accompanied by the reduction of the NifL-bound FAD cofactor, we studied the process of NifL reduction. Based on our findings in this study we propose that the FAD cofactor of NifL is reduced by a membrane-bound component of the anaerobic electron transport chain resulting in a reduced non-inhibitory conformation of NifL, which is membrane-associated. A first line of evidence was provided by biochemical analyses of the purified MBP-NifL protein. Spectral analysis clearly showed that in the absence of a redox mediator no change in absorbance of the flavoprotein was detectable upon the addition of $\mathrm{NADH} / \mathrm{H}^{+}$. However, the presence of methyl viologen or inside-out vesicles derived from $K$. pneumoniae cells grown under anaerobic conditions allowed NifL reduction by $\mathrm{NADH} / \mathrm{H}^{+}$(Figs. 15 and 16). This strongly indicates that in vivo NifL-bound FAD receives electrons from a membrane-bound oxidoreductase system. Two other lines of evidence derived from in vivo studies of nif regulation support this view. First, using $K$. pneumoniae or E. coli strains carrying null mutations of different membrane-bound oxidoreductases we showed that the absence of formate dehydrogenaseN or NADH:ubiquinone oxidoreductase in $K$. pneumoniae and the absence of NADH dehydrogenaseII in the heterologous $E$. coli system affects nif regulation dramatically. In the respective mutant strains nif induction was low, similar to induction levels under aerobic conditions, even though cells were grown under oxygen- and nitrogen-limitation (Table 8 and Fig. 19). These findings indicate that in the absence of the respective membrane-bound oxidoreductases the FAD-cofactor of NifL was not reduced at the cytoplasmic membrane 
resulting in cytoplasmic NifL, which inhibits NifA activity. Second, additional studies of nif induction in $K$. pneumoniae growing anaerobically on glycerol under nitrogen limitation revealed that the presence of nitrate as additional electron acceptor resulted in a significant decrease in nif induction (Table 9). It appears that under those energy-limited growth conditions, electrons of the reduced quinone pool are preferentially transferred onto nitrate allowing anaerobic respiration and energy conservation by the respiratory nitrate reductase (Tran et al. 1997, Unden and Bongaerts 1997). Thus, a high percentage of NifL protein does not receive electrons at the membrane and stays in its oxidized conformation in the cytoplasm inhibiting NifA activity resulting in decreased nif induction. Taking together, these data strongly indicate that more than one membrane-bound oxidoreductase system can provide electrons for NifL reduction under anaerobic conditions and we propose that under anaerobic conditions NifL receives electrons from the reduced quinone pool generated by different membrane-bound oxidoreductase systems. Reduction of the NifL-bound cofactor finally results in higher membrane-association of NifL, allowing cytoplasmic NifA to activate nif induction. The demonstration that the reduced soluble quinone derivative dimethylnaphthoquinone ( $\mathrm{E}^{\circ}=-240 \mathrm{mV}$, Krafft et al. 1995) is able to reduce the FAD cofactor of purified NifL in the absence of a redox mediator fully supports this model (Fig. 6). The reduction of NifL under anaerobic conditions at the cytoplasmic membrane by electrons derived from the reduced quinone pool rather than by a single specific membrane-bound enzyme is a particularly attractive model for several reasons. It explains (i) that the absence of different membrane-bound oxidoreductases in K. pneumoniae, which transfer electrons onto the quinone pool, results in the oxidized inhibitory conformation of NifL; and (ii) that NADH dehydrogenaseII in the heterologous $E$. coli system significantly affects nif regulation, although a homologous oxidoreductase appears not to be present in K. pneumoniae. Finally, reduction of NifL by the reduced quinone pool potentially allows the simultaneous signal integration of the cell's energy status for nif regulation.

In contrast to $K$. pneumoniae NifL no membrane-association for $A$. vinelandii NifL has been reported to date (Austin et al. 1994, Hill et al. 1996, Dixon, 1998). Thus, a different mechanism for the transduction of the oxygen signal to NifL is expected. In in vitro experiments $A$. vinelandii-NifL is reduced by $\mathrm{NADH} / \mathrm{H}^{+}$when catalyzed by the $E$. coli cytoplasmic flavoheme protein (HMP), which is proposed to be a global oxygen sensor or an oxidoreductase preventing cells from endogenous oxygen stress (Pool 1994, Macheroux et al. 1998, Stevanin et al. 2000;). However, the functional and physiological relevance of NifL reduction by HMP has not been demonstrated in vivo. It is currently hypothesized, that the 
reduction of $A$. vinelandii-NifL occurs non-specifically and dependant on the availability of reducing equivalents in the cell. Because of the relatively high redox potential of NifL, there are a number of electron donors and cytoplasmic NAD(P)H-dependent enzymes that could potentially be involved in reduction of NifL (Dixon 1998, Machereux et al. 1998). Interestingly, it was recently found that nitrogen fixation in the endophytic diazotroph Azoarcus sp. BH72 - belonging to the $\beta$-Proteobacteria - is also regulated by the coordinated activities of the homologous nifL and nifA gene products in response to environmental signals (Reinhold-Hurek et al. 1993, Egener and Reinhold-Hurek unpublished). However, it is currently not known whether NifL is membrane-associated under derepressing conditions or how reduction of the NifL-bound flavin cofactor occurs in the oxygen signal transduction.

\section{Nitrogen fixing $K$. pneumoniae M5a1 does not contain an NADH dehydrogenaseII}

homologous protein. E. coli contains two NADH:oxidoreductase systems. One enzyme, NADH:ubiquinone oxidoreductase (NDH-I) encoded by the nuo operon, couples $\mathrm{NADH} / \mathrm{H}^{+}$ oxidation to proton translocation and thus conserves the redox energy in a proton gradient (Weidner et al. 1993; Calhoun et al. 1993, Friedrich 2001). In contrast, the second enzyme, $\mathrm{NADH}$ dehydrogenaseII (NDH-II) encoded by $n d h$ does not couple the redox reaction to proton translocation (Matsushita et al. 1987, Calhoun et al. 1993). NDH-II is induced under aerobic conditions, whereas under anaerobic conditions NDH-II is apparently repressed by Fnr (Spiro 1989, Green and Guest 1994, Meng et al. 1997). When growing under anaerobic conditions in the presence of an electron acceptor for anaerobic respiration except conditions with high energy consumption, the coupling enzyme NDH-I is primarily expressed for higher ATP yields (Bongaerts et al. 1995, Tran et al. 1997, Wackwitz et al. 1999). However, expression patterns of the two enzymes can vary depending on specific requirements, e.g. conditions under which ATP yields are more important than growth rate (Unden et al. 2002). Contrary to E. coli we obtained strong evidence by Southern blot- and PCR-analysis that the nitrogen-fixing $K$. pneumoniae M5a1 strain does not exhibit a homologous NADHdehydrogenaseII in addition to the coupling NADH:ubiquinone oxidoreductase encoded by the nuo operon (Fig. 17). The finding that $\mathrm{NADH} / \mathrm{H}^{+}$oxidation activity in a $K$. pneumoniae M5a1 nuо mutant strain was neglectable compared to the oxidation rates of an E. coli nuo mutant strain (Falk-Krezesinski and Wolfe, 1998) further confirmed that K. pneumoniae M5a1 exhibits only a single NADH:oxidoreductase (Fig. 18). In contrast, the non nitrogenfixing strain K. pneumoniae subsp. pneumoniae exhibits both NADH:oxidoreductase systems (Fig. 17 and Sequencing Center, University of Washington, St. Louis, personal 
communication). These findings indicate that the presence of a single coupling NADH:oxidoreductase in $K$. pneumoniae M5a1 may be due to the high energy requirement for nitrogen fixation. We propose that the electrons transferred by the coupling enzyme $\mathrm{NADH}$ :ubiquinone oxidoreductase to the quinone pool are mainly transferred to fumarate reductase system for anaerobic fumarate respiration yielding in higher ATP yields.

Hypothetical model for oxygen control of nif regulation in $K$. pneumoniae. We have previously shown, that the global regulator Fnr is required for nif gene induction and proposed that Fnr transduces the signal of anaerobiosis towards NifL by activating genes the products of which reduce the FAD-cofactor of NifL (Grabbe et al. 2001b). We further demonstrated that under oxygen- and nitrogen-limited conditions NifL is membraneassociated and dissociates into the cytoplasm upon a shift to aerobic conditions (Klopprogge et al. 2002). In this study we obtained strong evidence that NifL is reduced at the cytoplasmic membrane by electrons derived from the quinone pool resulting in higher membrane affinity. In the presence of oxygen or nitrate as electron acceptors, NifL stays oxidized and is localized in the cytoplasm resulting in NifA inhibition. Thus, it is attractive to speculate that in $K$. pneumoniae M5a1 the membrane-associated oxidoreductases which transfer electrons to the quinone pool under anaerobic conditions are transcriptionally regulated by Fnr and thus are the downstream signal transductants following Fnr in the oxygen signal cascade.

As in E. coli the genes encoding formate dehydrogenaseN are transcribed in a Fnr dependent manner (Li and Steward 1992, Leonhartsberger et al. 2002) one can expect that expression of formate dehydrogenase $\mathrm{N}$ in $K$. pneumoniae is also transcriptionally controlled by Fnr. This is supported by sequence analysis of the $f d n G$ promoter upstream region, which indicates the presence of potential Fnr-boxes (data not shown). Transcription of the nuo operon in E. coli is regulated by molecular oxygen mainly through the transcriptional regulator ArcA which represses nио transcription under aerobic conditions (Bongaerts et al. 1995). Only small negative effects of Fnr onto nuo transcription have been reported under anaerobic growth, which might be due to effects of Fnr on arcA expression (Compan and Touati 1994, Bongaerts et al. 1995). However, as the nitrogen-fixing K. pneumoniae strain contains only a single NADH oxidizing enzyme, one can expect that the regulation of the nuo operon in $K$. pneumoniae differ significantly from the regulation of the nuo operon in E. coli. Based on preliminary sequence analysis of the promoter upstream regions of the nuoA gene, we speculate that in K. pneumoniae transcription of the nuo operon is up-regulated by Fnr under anaerobic conditions. Thus, in our current working model for oxygen signal transduction in $K$. 
pneumoniae we propose that under anaerobic conditions the primary oxygen sensor Fnr activates transcription of membrane-bound oxidoreductases leading to a reduced quinone pool, which provides electrons for NifL reduction. NifL reduction finally results in a higher membrane affinity of NifL and thus in a sequestration of NifL to the membrane, allowing cytoplasmic NifA to activate nif genes. However, as in E. coli under oxygen limitation the quinol oxidase (CydAB) is repressed by Fnr (Cotter et al. 1997, Govantes et al. 2000) we cannot completely rule out that the Fnr effect on nif induction in K. pneumoniae is based on derepression of a quinol oxidase in an $f n r$ mutant strain, which would also result in a decreased reduction of the quinone pool.

Concerning the nitrogen signal transduction it is not yet known how the primary nitrogen sensor GlnK transduces the nitrogen signal to the nif regulatory system in K. pneumoniae (He et al. 1998, Jack et al. 1999, Arcondeguy et al. 1999, Schmitz et al. 2002, Klopprogge et al. 2002). It is currently discussed that uridylylated GlnK transduces the signal of nitrogen limitation either by interacting with NifL or NifA, resulting in the dissociation of the inhibitory NifL/NifA complex. Thus, under nitrogen-limitation in the absence of oxygen, NifL would be able to receive electrons from the quinone pool and stay membrane-associated. We have previously shown that under anaerobic conditions a shift to nitrogen sufficiency results in dissociation of NifL from the cytoplasmic membrane, whereas the percentage of membrane-bound GlnK significantly increased (Klopprogge et al. 2002). Very recently, Merrick and coworkers provided evidence that upon a shift to nitrogen sufficiency deuridylylated GlnK in E. coli binds to the membrane in an AmtB-dependent manner (Coutts et al. 2002). If this is also the case in K. pneumoniae, it is attractive to speculate, that based on a shift to nitrogen sufficiency sequestration of non-uridylylated GlnK by AmtB would rapidly lower the cytoplasmic GlnK pool and thereby lowering the NifL fraction released from the inhibitory NifL/NifA complex by GlnK, which can be reduced at the membrane. 


\section{Conclusions}

In Klebsiella pneumoniae and Azotobacter vinelandii the nitrogen regulatory proteins NifL and NifA tightly control synthesis of nitrogen fixation genes in response to the environmental signals molecular oxygen and combined nitrogen. In this regulation the negative regulator NifL inhibits NifA transcriptional activity. Immunological studies, co-chromatography and complex formation analyses using the yeast two-hybrid system demonstrated that NifA interacts directly with NifL by protein-protein interaction (Henderson et al., 1989; Money et al. 1999 and 2001; Lei et al., 1999). This indicates that the signals of nitrogen sufficiency and/or molecular oxygen finally result in complex formation between NifL and NifA, which inhibits NifA activity and thus prevents unnecessary consumption of energy for nitrogen fixation. The inhibitor NifL is a flavoprotein, which regulates NifA activity depending on the reduction status of its N-terminally bound FAD-cofactor, allowing NifA transcriptional activity only under anaerobic conditions. Thus, the redox-sensitive FAD-cofactor appears to be involved in oxygen signal-transduction (Hill et al., 1996; Schmitz, 1997; Macheroux et al., 1998; Klopprogge and Schmitz, 1999). Both proteins, K. pneumoniae NifL and A. vinelandii NifL, were biochemically analyzed (Macheroux et al., 1998; Klopprogge and Schmitz, 1999), however, the physiological electron donor for NifL reduction is still discussed to date. In $A$. vinelandii, NifL reduction under anaerobic conditions apparently occurs unspecifically, depending on reducing equivalents available in the cell (Macheroux et al., 1998; Dixon, 1998). In K. pneumoniae, the relief of NifL inhibition under anaerobic conditions requires the presence of iron (Schmitz et al. 1996). As the NifL-protein does not contain iron or acid-labile sulphur (Schmitz, 1997), this finding indicates the involvement of an iron-containing protein in the oxygen-signaling mechanism, which is directly or indirectly required for NifL reduction. In Fig. 21 a model for oxygen signal-transduction in K. pneumoniae is presented. 


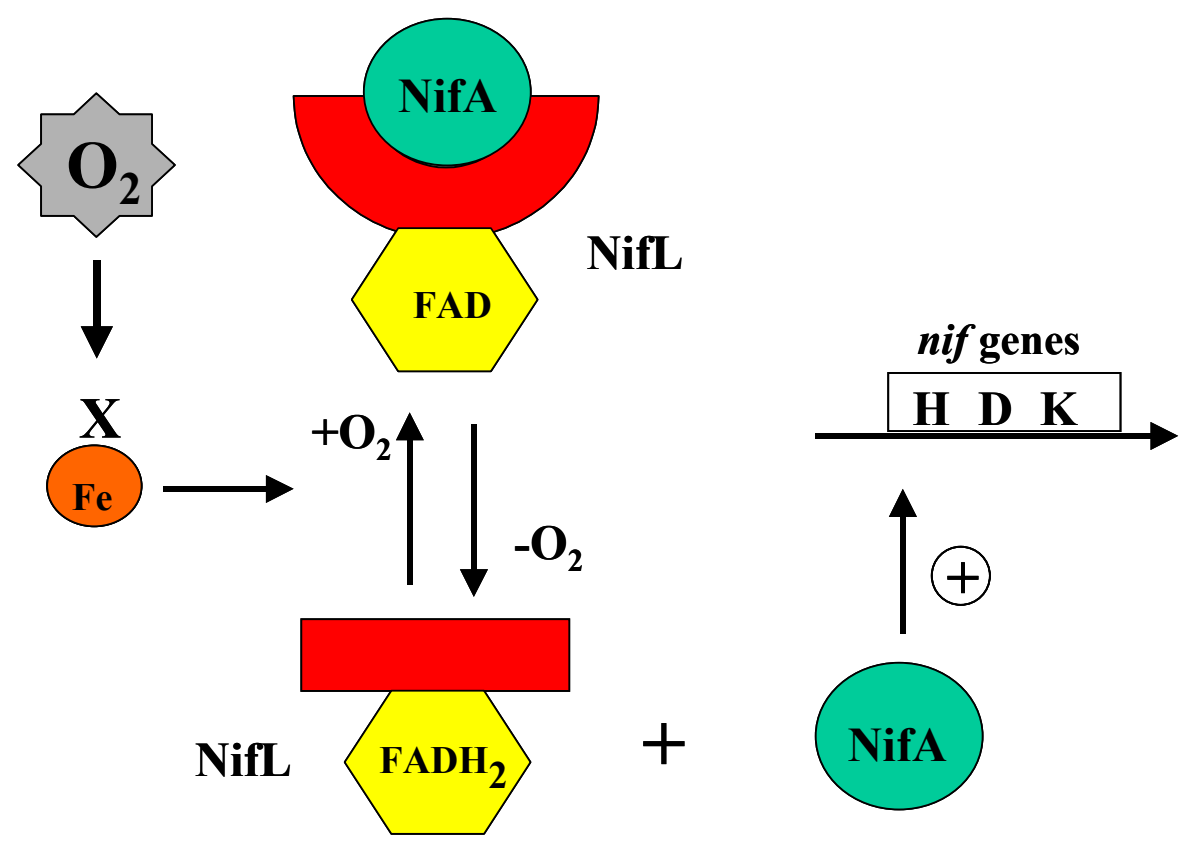

Fig. 21: Model for oxygen signal-transduction in K. pneumoniae.

The presence of an iron-containing protein in the oxygen signal transduction towards the flavoprotein NifL rises the question, whether a more general oxygen-sensing system might be involved in signal-transduction, which senses the redox status of the cell through an oxygensensitive iron-sulphur cluster. In Escherichia coli the Fnr-protein plays an overarching role as oxygen sensor in the cell and regulates transcription of respective genes. DNA-binding to regions upstream of the controlled genes (Fnr boxes) requires dimerization of Fnr monomers, which directly depends on the protein conformation induced by the [4Fe-4S]-cluster in each monomer (Ralph et al., 2001). After exposure to oxygen the [4Fe-4S]-clusters are destroyed and subsequent decay of the dimer into monomers results in an inactive form of Fnr containing an oxidized [2Fe-2S]-cluster (Green et al., 1996, Khoroshilova et al., 1997; Unden and Schirawski, 1997; Kiley and Beinert, 1998; Moore and Kiley, 2001). The gene product of K. pneumoniae fnr shows $98 \%$ homology to the Fnr-protein of E. coli. The K. pneumoniae protein contains the four conserved cystein residues in the N-terminus, which build up the iron-sulphur cluster (Grabbe et al., 2001a). We obtained significantly decreased amounts of 
iron in $K$. pneumoniae Fnr under aerobic conditions indicating that $K$. pneumoniae $\mathrm{Fnr}$ is sensing the redox status of the cell via a redox-sensitive [4Fe-4S]-cluster (Chapter 2, Grabbe et al., 2001a). The goal of this thesis was to analyze the potential role of Fnr in oxygen signaltransduction to the nif regulatory system.

We obtained strong evidence that in K. pneumoniae Fnr influences the oxygen-dependent nitrogen regulation by analyzing (i) NifA dependent nif induction in different fnr mutant strains (Chapter 3, Grabbe et al., 2001b), (ii) membrane-association of NifL in K. pneumoniae wild-type and $f n r$ mutant strains (Chapter 4, Klopprogge et al., 2002), and finally (iii) by studying the influence of different Fnr-dependent membrane-bound oxidoreductase systems on NifL modulated NifA activity (Chapter 5, Grabbe and Schmitz, 2002). In the following, each line of evidence is discussed in more detail.

(i) In the absence of Fnr, NifA mediated transcription of nif genes decreased significantly under nitrogen- and oxygen-limitation (Grabbe et al., 2001b). This indicates that the FAD moiety of NifL is not reduced, resulting in an inhibitory conformation of NifL. As we can rule out that the transcriptional activator Fnr provides electrons to reduce NifL, we postulate that Fnr transcriptionally controls genes, the products of which function to reduce the NifL-bound FAD-cofactor, resulting in a non-inhibitory conformation of NifL. Attractive candidates for the physiological electron donor are members of the anaerobic electron transport chain.

(ii) Localization of NifL in K. pneumoniae revealed that under anaerobic and nitrogen-limited conditions NifL is highly membrane-associated. Based on a shift to oxygen or nitrogen sufficiency in the absence of Fnr NifL is located mainly in the cytoplasm (Klopprogge et al., 2002). This indicates that NifL is apparently reduced during membrane-association by a membrane-bound oxidoreductase.

(iii) Studying the influence of different oxidoreductase systems on nif induction in $K$. pneumoniae we observed a remarkable decrease in NifA activity under oxygen and nitrogen limitation in the absence of a functional NADH:ubiquinone oxidoreductase or formate 
dehydrogenaseN. Analyzing nif induction in a heterologous E. coli system showed that in a $n d h$ mutant background NifL inhibition was not relieved under nitrogen and oxygen limitation (Grabbe and Schmitz, 2002). Based on these findings we conclude that under anaerobic conditions in $K$. pneumoniae the oxidoreductase systems NADH:ubiquinone oxidoreductase and formate dehydrogenase $\mathrm{N}$ generate a reduced quinone pool in the cytoplasmic membrane. In the absence of molecular oxygen, NifL contacts the membrane and receives electrons from the reduced quinone pool resulting in a non-inhibitory conformation of NifL. Subsequent analysis to verify the role of the reduced quinone pool towards the reduction of the NifLbound FAD-cofactor revealed, that reduced dimethylnaphthoquinone, a soluble quinone derivative, is able to function as electron donor for NifL in vitro (Grabbe and Schmitz, 2002). This further supports the model of NifL reduction by the reduced quinone pool, which is generated by those oxidoreductase systems. We further hypothesize that transcription of formate dehydrogenaseN and NADH:ubiquinone oxidoreductase is Fnr-dependent (Grabbe and Schmitz, 2002).

Current working model for oxygen-dependent control of NifA in $K$. pneumoniae. The results presented in this thesis are summarized in Fig. 22. Under anaerobic nitrogen-limiting conditions the NifL-protein of $K$. pneumoniae contacts the cytoplasmic membrane. This membrane-association is accompanied by reducing the FAD-cofactor with electrons from the reduced quinone pool, which apparently results in a membrane-associated NifL protein. Upon a shift to aerobiosis the FAD-cofactor is oxidized, NifL switches into an inhibitory conformation and dissociates from the membrane. The increased amount of inhibitory NifL in the cytoplasm interacts with NifA, resulting in a decrease of nif induction. Thus, we propose that sequestration of reduced NifL to the membrane under oxygen- and nitrogen-limitation creates a spatial gap between NifL and its target NifA, which is the regulatory mechanism for oxygen dependent control of NifA activity in K. pneumoniae. 


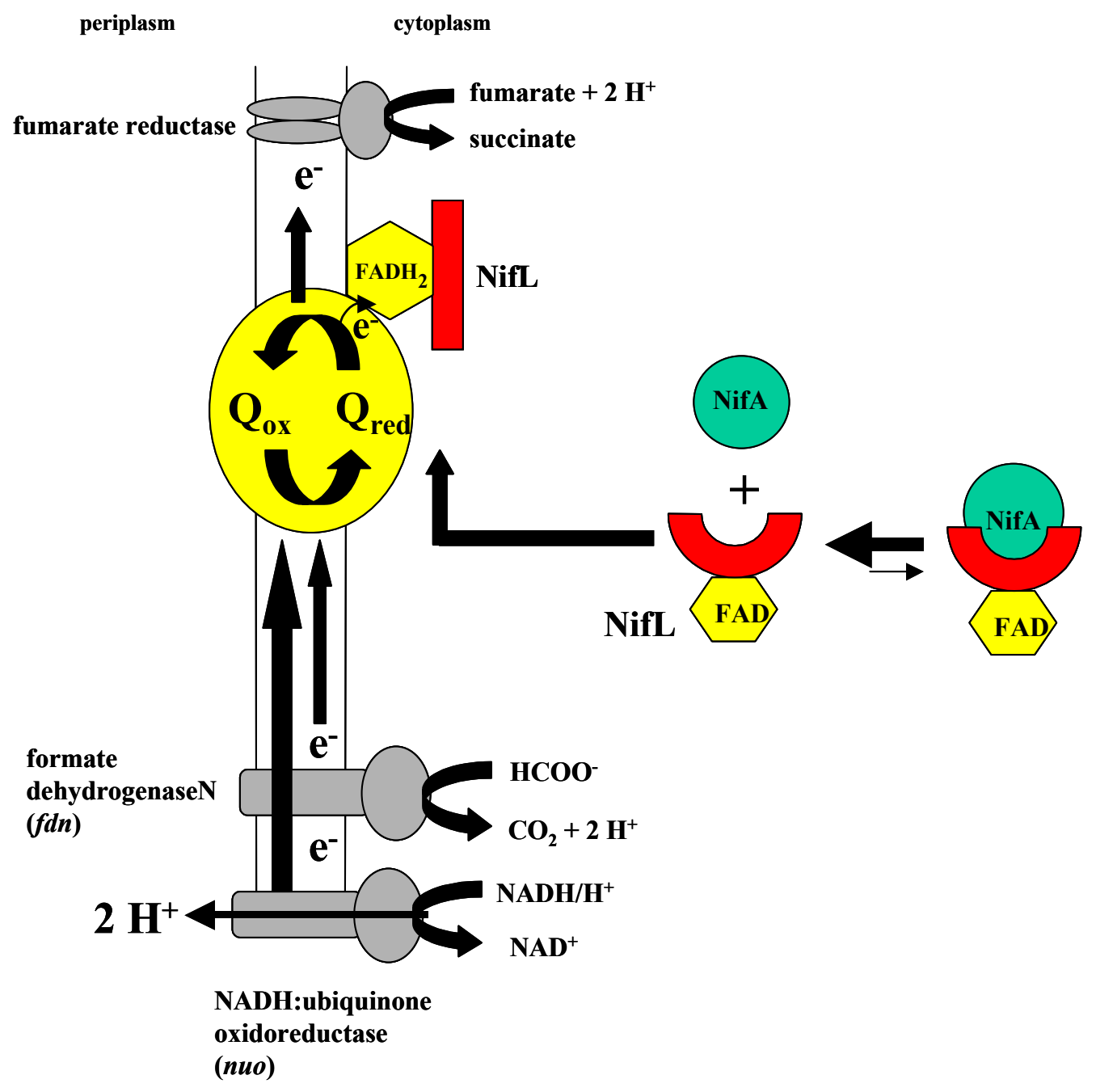

Fig. 22: Current working model for oxygen-dependent control of NifA activity in K. pneumoniae.

The nitrogen signal is mediated by the PII-like protein GlnK towards the nitrogen regulatory system in both organisms, K. pneumoniae and A. vinelandii. However, it is discussed that species-specific mechanisms are involved in the signaling cascade towards the nitrogen fixation regulon. In $A$. vinelandii non-uridylylated GlnK enhances NifL inhibitory functions under nitrogen sufficiency (Little et al., 2000), whereas in K. pneumoniae GlnK is required for relief of NifL inhibition (He et al., 1998; Jack et al., 1999). Recently, it was demonstrated that in E. coli and A. vinelandii after a shift to nitrogen sufficiency non-uridylylated GlnK is membrane-associated by binding to the ammonium transporter AmtB (Coutts et al., 2002). This is contradictory to the model Dixon and coworkers proposed, that under nitrogen 
sufficiency in $A$. vinelandii unmodified GlnK interacts with NifL in the cytoplasm and activates NifL inhibitory functions (Little et al., 2002). In K. pneumoniae, GlnK antagonizes NifL inhibitory function towards NifA activity, but uridylylation of GlnK is apparently not required for relief of NifL inhibition (He et al. 1998; Jack et al., 1999; Arcondeguy et al., 1999). If this is also the case in K. pneumoniae it is attractive to speculate that under nitrogenand oxygen-limitation GlnK in its uridylylated form remains located in the cytoplasm preventing complex formation between NifL and NifA. Upon a shift to nitrogen sufficiency non-uridylylated GlnK binds to AmtB and stays membrane-associated. As a consequence, NifL interacts with NifA inhibiting NifA activity.

\section{Further studies}

Due to the fact that the reduced quinone pool, generated by oxidoreductase systems of the anaerobic respiratory chain, is responsible for NifL reduction, the localization of NifL in the NADH:ubiquinone oxidoreductase and the formate dehydrogenaseN mutant has to be examined. Second, the proposed Fnr-dependency of those oxidoreductase systems has to be analyzed. Finally, it has to be determined, whether the dramatically reduced NifA activity in the $K$. pneumoniae $f d n$ and nuo mutants is also a consequence of reduced energy charge in the cell or other metabolic signals. 


\section{References}

Ackrell, B.A. 2000. Progress in understanding structure-function relationships in respiratory chain complex II. FEBS Lett. 466: 1-5.

Arcondeguy, T., Jack, R., and Merrick, M. 2001. The PII signal transduction proteins: pivotal players in microbial nitrogen control. Microbiol. Mol. Biol. Rev. 65: 80-105.

Arcondeguy, T., Lawson, D., and Merrick, M. 2000. Two residues in the T-loop of Klebsiella pneumoniae GlnK determine NifL-dependent nitrogen control of nif gene expression. J. Biol. Chem. 275(49): 38452-6.

Arcondeguy, T., van Heeswijk, W.C., and Merrick, M. 1999. Studies on the roles of GlnK and GlnB in regulating Klebsiella pneumoniae NifL-dependent nitrogen control. FEMS Microbiol. Lett. 2: 263-70.

Arnott, M., Sidoti, C., Hill, S., and Merrick, M. 1989. Deletion analysis of the nitrogen fixation regulatory gene nifL of Klebsiella pneumoniae. Arch. Microbiol. 151: 180-82.

Arsene, F., Kaminski, P.A., and Elmerich, C. 1999. Control of Azospirillum brasilense NifA activity by $\mathrm{P}(\mathrm{II})$ : effect of replacing Tyr residues of the NifA N-terminal domain on NifA activity. FEMS Microbio. Lett. 2: 339-43.

Ausubel, F., Brent, R., Kingston, R.E., Moore, D.D., Seidmann, J.G., Smith, J.A., and Struhl, K. 1987. Current protocols in molecular biology. John Wiley and Sons, New York.

Atkinson, M.R., and Ninfa, A.J. 1999. Characterization of the GlnK protein of Escherichia coli. Mol. Microbiol. 32: 301-13.

Atkinson, M.R, and Ninfa, A.J. 1998. Role of the GlnK signal transduction protein in the regulation of nitrogen assimilation in Escherichia coli. Mol. Microbiol. 29(2): 431-47.

Atkinson, M.R., Kamberov, E.S., Weiss, R.L. and Ninfa, A.J. 1994. Reversible uridylylation of the Escherichia coli PII signal transduction protein regulates its ability to stimulate the dephosphorylation of the transcription factor nitrogen regulator I (NRI or NtrC). J. Biol. Chem. 269(45): 28288-93.

Austin, S., Buck, M., Cannon, W., Eydmann, T., and Dixon, R. 1994. Purification and in vitro activities of the native nitrogen fixation control proteins NifA and NifL. J. Bacteriol. 176: 3460-55.

Austin, S., Henderson, N. and Dixon R. 1990. Characterisation of the Klebsiella pneumoniae nitrogen-fixation regulatory proteins NIFA and NIFL in vitro. Eur. J. Biochem. 187(2): 353-60. 
Barrett, J., Ray, P., Sobczyk, A., Little, R., and Dixon, R. 2001. Concerted inhibition of the transcriptional activation functions of the enhancer-binding protein NIFA by the antiactivator NIFL. Mol. Microbiol. 39(2): 480-93.

Bauer, C.E., Elsen, S., and Bird, T.H. 1999. Mechanisms for redox control of gene expression. Annu. Rev. Microbiol. 53: 495-523.

Beinert, H., and Kiley, P.J. 1998. Oxygen sensing by the global regulator, FNR: the role of the iron-sulfur cluster. FEMS Microbiol. Rev. 22(5): 341-52.

Bergmayer, H.U.1983. Methods of enzymatic analysis Volume II. 3. Auflage, Verlag Chemie Weinheim.

Bibikov, S.I., Biran, R., Rudd, K.E., and Parkinson, J.S. 1997. A signal transducer for aerotaxis in Escherichia coli. J. Bacteriol. 179(12): 4075-9.

Blanco, G., Drummond, M., Woodley, P., and Kennedy, C. 1993. Sequence and molecular analysis of the nifL gene of Azotobacter vinelandii. Mol. Microbiol. 9: 869-80.

Bongaerts, J., Zoske, S., Weidner, U., and Unden, G. 1995. Transcriptional regulation of the proton translocating NADH dehydrogenase genes (nuоA-N) of Escherichia coli electron acceptors, electron donors and gene regulators. Mol. Microbiol. 16(3): 521-34.

Bott, M., Meyer, M., and Dimroth, P. 1995. Regulation of anaerobic citrate metabolism in Klebsiella pneumoniae. Mol. Microbiol. 18: 533-46.

Bradford, M.M. 1976. A rapid and sensitive method for the quantitation of microgram quantities of protein utilizing the principle of protein-dye binding. Anal. Biochem. 72: 248-54.

Brown, E.D. and Wood, J.M. 1993. Nucleotide Conformational change and membrane association of the PutA protein are coincident with reduction of its FAD cofactor by proline. J. Biol. Chem. 268(12): 8972-9.

Buck, M., Cannon, W., and Woodcock, J. 1987. Transcriptional activation of the Klebsiella pneumoniae nitrogenase promoter may involve DNA loop formation. Mol. Microbiol. 1: $243-49$.

Burgess, B.K., and Lowe, D.J. 1996. Mechanism of molybdenum nitrogenase. Chem. Rev. 96: 2983-3011.

Calhoun, M.W., Oden, K.L., Gennis, R.B., de Mattos, M.J., and Neijssel, O.M. 1993. Energetic efficiency of Escherichia coli: effects of mutations in components of the aerobic respiratory chain. J, Bacteriol. 175(10): 3020-5.

Cline, J.D. 1969. Spectromphotometric determination of hydrogen sulfide in natural waters. Limnol. Oceanogr. 14: 454-58. 
Compan, I., and Touati, D. 1994. Anaerobic activation of $\operatorname{arcA}$ transcription in Escherichia coli: roles of Fnr and ArcA. Mol, Microbiol, 11(5): 955-64.

Cotter, P.A., Melville, S.B., Albrecht, J.A., and Gunsalus, R.P. 1997. Aerobic regulation of cytochrome d oxidase (cydAB) operon expression in Escherichia coli: roles of Fnr and ArcA inrepression and activation. Mol, Microbiol, 25(3): 605-15.

Coutts, G., Thomas, G., Blakeley, D., and Merrick, M. 2002. Membrane sequestration of the signal transduction protein GlnK by the ammónium transproter AmtB. EMBO Journal 21(4):536-45.

Cruz Ramos, H., Boursier, L., Moszer, I., Kunst, F., Danchin, A., and Glaser, P. 1995. Anaerobic transcription activation in Bacillus subtilis: identification of distinct FNRdependent and -independent regulatory mechanisms. EMBO J. 14: 5984-94.

Daesch, G., and Mortenson, L.E. 1972. Effect of ammonia on the synthesis and function of the $\mathrm{N}_{2}$-fixing enzyme systeme in Clostridium pasteurianum. J. Bacteriol. 110: 103-9.

Dean, D.R., and Jacobson, M.R. 1992. Biochemical genetics of nitrogenase In: Biological nitrogen fixation. G. Stacey, R.H. Burris and H.J. Evans, eds. Chapman \& Hall, New York . p. 763-834.

DeLorenzo, V., Herrero, M., Jakubzik, U., and Timmis, K.N. 1990. Mini-Tn5 Transposon Derivatives for Insertion Mutagenesis, Promoter Probing, and Chromosomal Insertion of Cloned DNA in Gram-Negative Eubacteria. J. Bacteriol. 172(11): 6568-72.

Deppenmeier, U., Johann, A., Hartsch, T., Merkl, R., Schmitz, R.A., Lienard, T., Henne, A., Martinez-Arias, R., Wiezer, A., Jacobi, C., Brüggemann, H., Christmann, A., Bäumer, S., Bömeke, M., Steckel, S., Bhattacharyya, A., Lykidis, A., Overbeek, R., Klenk, H.-P., Gunsalus, R.P., Fritz, H.-J., and Gottschalk, G. (2002). The genome of Methanosarcina mazei: evidence for lateral gene transfer between Bacteria and Archaea. J. Mol. Microbiol. Biotechnol. (ahead of print).

Devereux, J., Haeberli, P., and Smithies, O. 1984. A comprehensive set of sequence analysis programs for the VAX. Nucleic Acids Res. 12: 387-95.

Dixon, R. 1998. The oxygen-responsive NifL-NifA complex: a novel two-component regulatory system controlling nitrogenase synthesis in gamma-proteobacteria. Arch. Microbiol. 169: 371-80.

Dixon, R.A., Cannon, F., and Kondorosi, A. 1976. Construction of a P plasmid carrying nitrogen fixation genes from Klebsiella pneumoniae. Nature 260: 268-71. 
Drummond, M.H., Contreras, A., and Mitchenall, L.A. 1990. The function of isolated domains and chimaeric proteins constructed from the transcriptional activators NifA and NtrC of Klebsiella pneumoniae. Mol. Microbiol. 4: 29-37.

Drummond, M.H., and Wootton, J.C. 1987. Sequence of nifL from Klebsiella pneumoniae: mode of action and relationship to two families of regulatory proteins. Mol. Microbiol. $1: 37-44$.

Falk-Krzesinski, H.J., and Wolfe, A. 1998. Genetic Analysis of the nuo Locus, Which Encodes the Proton-Translocating NADH Dehydrogenase in Escherichia coli. J. Bacteriol. 180(5): 1174-84.

Filser, M., Merrick, M., and Cannon, F. 1983, Cloning and characterization of nifLA regulatory mutations from Klebsiella pneumoniae. Mol. Gen. Genet. 191: 485-91.

Fischer, H.M. 1994. Genetic regulation of nitrogen fixation in rhizobia. Microbiol. Reviews 58: $352-86$.

Fischer, H.M. 1996. Environmental regulation of rhizobial symbiotic nitrogen fixation genes. Trends Microbiol. 4: 317-20.

Fish, W.W. 1988. Rapid colorimetric micromethod for the quantitation of complexed iron in biological samples. Methods Enzymol. 158: 357-64.

Flückiger, R., Paz, M.A. and Gallop, P.M. 1995. Redox-cycling detection of dialyzable pyrroloquinoline quinone and quinoproteins. Methods in Enzymology 258: 140-49.

Forchhammer, K., and Hedler, A. 1997. Phosphoprotein PII from cyanobacteria--analysis of functional conservation with the PII signal-transduction protein from Escherichia coli. Eur. J. Biochem. 244(3): 869-75.

Friedrich, T. 2001. Complex I: a chimaera of a redox and conformation-driven proton pump? J. Bioenerg. Biomembr. 33(3): 169-77.

Friedrich, T., Hofhaus, G., Ise, W., Nehls, U., Schmitz, B., and Weiss, H. 1989. A small isoform of NADH:ubiquinone oxidoreductase (complex I) without mitochondrially encoded subunits is made in chloramphenicol-treated Neurospora crassa. Eur J Biochem. 180(1): 173-80.

Galagan, J.E., Nusbaum, C., Roy, A., Endrizzi, M.G., Macdonald, P., FitzHugh, W., Calvo, S., Engels, R., Smirnov, S., Atnoor, D., Brown, A., Allen, N., Naylor, J., StangeThomann, N., DeArellano, K., Johnson, R., Linton, L., McEwan, P., McKernan, K., Talamas, J., Tirrell, A., Ye, W., Zimmer, A., Barber, R.D., Cann, I., Graham, D.E., Grahame, D.A., Guss, A.M., Hedderich, R., Ingram-Smith, C., Kuettner, H.C., Krzycki, J.A., Leigh, J.A., Li, W., Liu, J., Mukhopadhyay, B., Reeve, J.N., Smith, K., Springer, 
T.A., Umayam, L.A., White, O., White, R.H., Conway de Macario, E., Ferry, J.G., Jarrell, K.F., Jing, H., Macario, A.J., Paulsen, I., Pritchett, M., Sowers, K.R., Swanson, R.V., Zinder, S.H., Lander, E., Metcalf, W.W., and Birren, B. 2002. The genome of M. acetivorans reveals extensive metabolic and physiological diversity. Genome Res. 12(4): 532-42.

Govantes, F., Orjalo, A.V., and Gunsalus, R.P. 2000. Interplay between three global regulatory proteins mediates oxygen regulation of the Escherichia coli cytochrome doxidase (cydAB) operon. Mol. Microbiol. 38(5): 1061-73.

Govantes, F., Andújar, E., and Santero, E. 1998. Mechanism of translational coupling in the nifLA operon of Klebsiella pneumoniae. EMBO J. 17: 2368-77.

Gostick, D.O., Green, J., Irvine, A.S., Gasson, M.J., and Guest, J.R. 1998. A novel regulatory switch mediated by the FNR-like protein of Lactobacillus casei. Microbiology 144: 70517.

Grabbe, R., and Schmitz, R.A. 2002. Oxygen Control of nif Gene Expression in Klebsiella pneumoniae is dependent on NifL reduction at the cytoplasmic membrane by electrons derived from the reduced quinone pool. submitted.

Grabbe, R., Kuhn, A., and Schmitz, R.A. 2001a. Cloning, sequencing and characterization of Fnr from Klebsiella pneumoniae. Antonie Van Leeuwenhoek 79: 319-26.

Grabbe, R., Klopprogge, K., and Schmitz, R.A. 2001b. Fnr is Required for NifL-dependent oxygen control of nif gene expression in Klebsiella pneumoniae. J. Bacteriol. 183: 1385-93.

Green, J., Bennett, B., Jordan, P., Ralph, E.T., Thomson, A.J., and J.R. Guest. 1996. Reconstitution of the [4Fe-4S] cluster in FNR and demonstration of the aerobic-anaerobic transcription switch in vitro. Biochem. J. 316: 887-92.

Green, J., and Guest, J.R. 1994. Regulation of transcription at the $n d h$ promoter of Escherichia coli by Fnr and novel factors. Mol. Microbiol. 12(3): 433-44.

Gutierrez, D., Hernando, Y., Palacios, J.M., Imperial, J., and Ruiz-Argueso, T. 1997. FnrN controls symbiotic nitrogen fixation and hydrogenase activities in Rhizobium leguminosarum biovar viciae UPM791. J. Bacteriol. 179: 5264-70.

Gunsalus, R.P., and Park, S.J. 1994. Aerobic-anaerobic gene regulation in Escherichia coli: control by the ArcAB and Fnr regulons. Res. Microbiol. 145: 437-50.

Halbleib, C.M., and Ludden, P.W. 2000. Regulation of biological nitrogen fixation. J. Nutr. 130: $1081-84$. 
He, L., Soupene, E., Ninfa, A., and Kustu, S. 1998. Physiological role for the GlnK protein of enteric bacteria: relief of NifL inhibition under nitrogen-limiting conditions. J. Bacteriol. 180: 6661-67.

He, L., Soupene, E., and Kustu, S. 1997. NtrC is required for control of Klebsiella pneumoniae NifL activity. J. Bacteriol. 179: 7446-55.

Henderson, N., Austin, S., and Dixon, R. A. 1989. Role of metal ions in negative regulation of nitrogen fixation by the nifL gene produkt from Klebsiella pneumoniae. Mol. Gen. Genet. 216: 484-91.

Hill, S., Austin, S., Eydmann, T., Jones, T., and Dixon, R. 1996. Azotobacter vinelandii NifL is a flavoprotein that modulates transcriptional activation of nitrogen-fixation genes via a redox-sensetive switch. Proc. Natl. Acad. Sci. USA 93: 2143-48.

Hill, S. 1985. Redox regulation of enteric nif expression is independent of the fnr gene product. FEMS Microbiol. Lett. 29: 5-9.

Hill, S., Kennedy, C., Kavanagh, E., Goldberg, R., and Hanau, R. 1981. Nitrogen fixation gene (nifL) involved in oxygen regulation of nitrogenase synthesis in Klebsiella pneumoniae. Nature 290: 424-26.

Hoover, T.R., Santero, E., Porter, S., and Kustu, S. 1990. The integration host factor stimulates interaction of RNA polymerase with NIFA, the transcriptional activator for nitrogen fixation operons. Cell 63: 11-22.

Hoppert, M., Holzenburg, A. 1998. Electron Microscopy in Microbiology. Bios.. Scientific. Publ., Oxford, UK.

Howard, J.B., and Rees, D.C. 1996. Structural basis of biological nitrogen fixation. Chem. Rev. 96: 2965-82.

Imlay, J.A. 1995. A metabolic enzyme that rapidly produces superoxide, fumarate reductase of Escherichia coli. J. Biol. Chem. 270(34): 19767-77.

Inoue, H., H. Nojima, and Okayama, H. 1990. High efficiency transformation of Escherichia coli with plasmids. Gene 9: 23-28.

Jack, R., DeZamaroczy, M., and Merrick, M. 1999. The signal transduction protein GlnK is required for NifL-dependent nitrogen control of nif gene expression in Klebsiella pneumoniae. J. Bacteriol. 4: 1156-62.

Jayaraman, P.S., Gaston, K.L., Cole, J.A., and Busby, S.J.W. 1988. The nirB promoter of Escherichia coli: location of nucleotide sequences essential for regulation by oxygen, the FNR protein and nitrite. Mol. Microbiol. 2: 527-30. 
Jiang, P., Peliska, J.A., and Ninfa, A.J. 1998. Enzymological characterization of the signaltransducing uridylyltransferase/uridylyl-removing enzyme (EC2.7.7.59) of Escherichia coli and its interaction with the PII protein. Biochemistry. 37: 12782-94.

Khoroshilova, N., Popescu, C., Munck, E., Beinert, H., and Kiley, P.J. 1997. Iron-sulfur cluster disassembly in the FNR protein of Escherichia coli by $\mathrm{O}_{2}$ : [4Fe-4S] to [2Fe-2S] conversion with loss of biological activity. Proc. Natl. Acad. Sci. USA. 94: 6087-92.

Kiley, P.J., and Beinert, H. 1998. Oxygen sensing by the global regulator, FNR: the role of the iron-sulfur cluster. FEMS Microbiol. Rev. 5: 341-52.

Klinger, A., Schirawski, J., Glaser, P., and Unden, G. 1998. The fnr gene of Bacillus licheniformis and the cysteine ligands of the C-terminal FeS cluster. J. Bacteriol. 180: 3483-5.

Klopprogge, K., Grabbe, R., Hoppert, M., and Schmitz, R.A. 2002. Membrane association of Klebsiella pneumoniae NifL is affected by molecular oxygen and combined nitrogen. Arch. Microbiol. 177(3): 223-34.

Klopprogge, K., and Schmitz, R.A. 1999. NifL of Klebsiella pneumoniae: redox characterization in relation to the nitrogen source. Biochim. Biophys. Acta 1431: 46270.

Krafft, T., Gross, R., and Kröger, A. 1995. The function of Wolinella succinogenes psr genes in electron transport with polysulphide as the terminal electron acceptor. Eur. J. Biochem. 230(2): 601-6.

Krebs, W., Steuber, J., Gemperli, A.C., and Dimroth, P. 1999. Na+ translocation by the NADH:ubiquinone oxidoreductase (complex I) from Klebsiella pneumoniae. Mol. Microbiol. 33(3): 590-8.

Laemmli, U.K. 1970. Cleavage of the structural proteins during the assembly of the head of the Bacteriophage T4. Nature 227: 680-85.

Lazazzera, B.A., Beinert, H., Khoroshilova, N., Kennedy, M.C., and Kiley, P.J. 1996. DNA binding and dimerization of the Fe-S-containing FNR protein from Escherichia coli are regulated by oxygen. Biol. Chem. 271: 2762-68.

Lee, H.S., Berger, D.K., and Kustu, S. 1993a. Activity of purified NIFA, a transcriptional activator of nitrogen fixation genes. Proc. Natl. Acad. Sci. U S A 90: 2266-70.

Lee, H. S., Narberhaus, F., and Kustu, S. 1993b. In vitro activity of NifL, a signal transduction protein for biological nitrogen fixation. J. Bacteriol. 175: 7683-88. 
Lei, S., Pulakat, L., and Gvani, N. 1999. Genetiv analysis of nif regulatory genes by utilizing the yeast two-hybrid system detected formation of a NifL-NifA complex that is implicated in regulated expression of nif genes. J. Bacteriol. 181: 6535-39.

Leonhartsberger, S., Korsa, I., and Boeck, A. 2002. The molecular biology of formate metabolism in Enterobacteria. J. Mol. Microbiol. Biotechnol. 4(3): 269-76.

Li, J., and Steward, V. 1992. Localization of upstream sequence elements required for nitrate and anaerobic induction of $f d n$ (Formate Dehydrogenase-N) operon expression in Escherichia coli K-12. J. Bacteriol. 174(15): 4935-42.

Little, R., Colombo, V., Leech, A., and Dixon, R. 2002. Direct interaction of the NifL regulatory protein with the GlnK signal transducer enables the Azotobacter vinelandii NifL-NifA regulatory system to respond to conditions replete for nitrogen. J. Biol. Chem. 277(18): 15472-81.

Little, R., Reyes-Ramirez, F., Zhang, Y., van Heeswijk, W.C., and Dixon, R. 2000. Signal transduction to the Azotobacter vinelandii NIFL-NIFA regulatory system is influenced directly by interaction with 2-oxoglutarate and the PII regulatory protein. EMBO J. 19: 6041-50.

Lobo, A.L., and Zinder, S.H. 1992. Nitrogen fixation by methanogenic bacteria. In: Biological nitrogen fixation. G. Stacey, R.H. Burris and H.J. Evans, eds. Chapman \& Hall, New York. p. 736-62.

Macheroux, P., Hill, S., Austin, S., Eydmann, T., Jones, T., Kim, S.O., Poole, R., and Dixon, R. 1998. Electron donation to the flavoprotein NifL, a redox-rensing transcriptional regulator. Biochem. J. 332: 413-19.

MacNeil, D., Zhu, J., and. Brill, W.J. 1981. Regulation of nitrogen fixation in Klebsiella pneumoniae: isolation and characterization of strains with nif-lac fusions. J. Bacteriol. 145: 348-57.

Maloy, S. 1987. The proline utilization operon, in: Neidhardt, F.C., Ingraham, J.L, Low, K.B., Magasanik, B., Schaechter M. and Umbarger H.E. (Eds.), Salmonella typhimurium: Cellular and molecular biology, American Society for Microbiology, Washington, DC: 1513-19.

Manodori, A., G. Cecchini, I. Schröder, R. P. Gunsalus, M. T. Werth, and M. K. Johnson. 1992. [3Fe-4S] to [4Fe-4S] cluster conversion in Escherichia coli fumarate reductase by site-directed mutagenesis. Biochemistry 31: 2703-12.

Matsushita, K., Ohnishi, T., and Kaback, H.R. 1987. NADH-ubiquinone oxidoreductases of the Escherichia coli aerobic respiratory chain. Biochemistry. 26(24): 7732-7. 
Mayer, D., Schlensog, V., and Böck, A. 1995. Identification of the transcriptional activator controlling the butanediol fermentation pathway in Klebsiella terrigena. J. Bacteriol. 177: 5261-69.

Meletzus, D., Rudnick, P., Doetsch, N., Green, A., and Kennedy, C. 1998. Characterization of the $g \ln K$-amtB operon of Azotobacter vinelandii. J. Bacteriol. 12: 3260-64.

Melville, S.B., and Gunsalus, R.P. 1996. Isolation of an oxygen-sensitive FNR protein of Escherichia coli: interaction at activator and repressor sites of FNR-controlled genes. Proc. Natl. Acad. Sci. USA. 93: 1226-31.

Melville, S.B., and R.P. Gunsalus. 1990. Mutations in $f n r$ that alter anaerobic regulation of electron transport-associated genes in Escherichia coli. J. Biol.Chem. 265: 18733-36.

Meng, W., Green, J., and Guest, J.R. 1997. FNR-dependent repression of $n d h$ gene expression requires two upstream FNR-binding sites. Microbiology. 143 ( Pt 5): 1521-32.

Merrick, M., Hill, S. Hennecke, H., Hahn, M. Dixon, R., and Kennedy, C. 1982. Repressor properties of the nifL gene product in Klebsiella pneumoniae. Mol. Gen. Genet. 185: $75-81$.

Money, T., Barrett, J., Dixon, R., and Austin, S. 2001. Protein-protein interactions in the complex between the enhancer binding protein NIFA and the sensor NIFL from Azotobacter vinelandii. J. Bacteriol. 183(4): 1359-68.

Money, T., Jones, T., Dixon, R., and Austin, S. 1999. Isolation and properties of the complex between the enhancer binding protein NifA and the sensor NifL. J. Bacteriol. 181: 446168.

Monteiro, R.A., Souza, E.M., Yates, M.G., Pedrosa, F.O., and Chubatsu, L.S. 1999a. In-trans regulation of the N-truncated-NIFA protein of Herbaspirillum seropedicae by the N-terminal domain. FEMS Microbio. Lett. 2: 157-61.

Monteiro, R.A., Souza, E.M., Funayama, S., Yates, M.G., Pedrosa, F.O., and Chubatsu, L.S. 1999b. Expression and functional analysis of an N-truncated NifA protein of Herbaspirillum seropedica. FEBS Lett. 447: 283-86.

Moore, L.J, and Kiley, P.J. 2001. Characterization of the dimerization domain in the FNR transcription factor. J. Biol. Chem. 276(49): 45744-50.

Morett, E., and Segovia, L. 1993. The sigma 54 bacterial enhancer-binding protein family: mechanism of action and phylogenetic relationship of their functional domains. J. Bacteriol. 175: 6067-74. 
Morett, E., and Buck, M. 1989. In vivo studies on the interaction of RNA polymerase- 54 with the Klebsiella pneumoniae and Rhizobium meliloti nifH promoters. J. Mol. Biol. 210: 65-77.

Morett, E., and Buck, M. 1988. NifA-dependent in vivo protection demonstrates that the upstream activator sequence of nif promoters is a protein binding site. Proc. Natl. Acad. Sci. USA 85: 9401-05.

Mott, J.E., Grant, R.A., Ho, Y.S., and Platt, T. (1985) Maximizing gene expression from plasmid vectors containing the lambda PL promoter: Strategies for overproducing transcription termination factor- . Proc. Natl. Acad. Sci. USA 82, 88-92.

Muro-Pastor, A., Ostrovsky, P., and Maloy, J. 1997. Regulation of gene expression by repressor localization: biochemical evidence that membrane and DNA binding by the PutA protein are mutually exclusive. J. Bacteriol. 179(8): 2788-91.

Nakano, Y., Y. Yoshida, Y. Yamashita, and T. Koga. 1995. Construction of a series of pACYC-derived plasmid vectors. Gene 162: 157-58.

Narberhaus, F., Lee, H.-S., Schmitz, R.A., He, L., and Kustu, S. 1995. The C-terminal domain of NifL is sufficient to inhibit NifA activity. J. Bacteriol. 177: 5078-87.

Ostrovsky de Spicer, P., O'Brien, K. and Maloy, S. 1991. Regulation of proline utilization in Salmonella typhimurium: a membrane-associated dehydrogenase binds DNA invitro. J. Bacteriol. 173(1): 211-9.

Parkinson, J.S., and Kofoid, E.C. 1992. Communication modules in bacterial signaling proteins. Annu. Rev. Genet. 26: 71-112.

Poole, R. K. 1994. Oxygen reactions with bacterial oxidases and globins: binding, reduction and regulation. Antonie van Leuwenhoek 65: 289-310.

Prentki, P., and Kirsch, H. M. 1984. In vitro insertional mutagenesis with a selectable DNA fragment. Gene 29: 303-13.

Ralph, E.T., Scott, C., Jordan, P.A., Thomson, A.J., Guest, J.R., and Green, J. 2001. Anaerobic acquisition of [4FE 4S] clusters by the inactive $\mathrm{FNR}(\mathrm{C} 20 \mathrm{~S})$ variant and restoration of activity by second-site amino acid substitutions. Mol. Microbiol. 39(5): 1199-211.

Rebbapragada, A., Johnson, M.S., Harding, G.P., Zuccarelli, A.J., Fletcher, H.M., Zhulin, I.B., and Taylor, B.L. 1997. The Aer protein and the serine chemoreceptor Tsr independently sense intracellular energy levels and transduceoxygen, redox, and energy signals for Escherichia coli behavior. Proc. Natl. Acad. Sci. U S A. 94(20): 10541-6. 
Rees, D.C., and Howard, J.B. 1999. Structural bioenergetics and energy transduction mechanisms. J. Mol. Biol. 293: 343-50.

Reinhold-Hurek, B., Hurek, T., Gillis, M., Hoste, B., Vancanneyt, M., Kersters, K., and Deley, J. 1993. Azoarcus gen. nov., nitrogen-fixing proteobacteria associated withroots of kallar grass (Leptochloa fusca (L.) Kunth), and description of two species, Azoarcus indigens sp. nov. and Azoarcus communis sp. nov. Int. J. Syst. Bacteriol. 43: 574-84.

Reyes-Ramirez, F., Little, R., and Dixon, R. 2001. Role of Escherichia coli nitrogen regulatory genes in the nitrogen response of the Azotobacter vinelandii NifL-NifA complex. J. Bacteriol. 183(10): 3076-82.

Reyes-Ramirez, F., Little, R., Hill, S., van Heeswijk, W., and Dixon, R. 2000. Regulation of Azotobacter vinelandii NifA activity by NifL: role of PII-like proteins in nitrogen sensing. In:Nitrogen fixation; from molecules to crop productivity. F.O. Pedrosa, M. Hungria, M.G. Yates and W.E. Newton, eds.Kluwer Academic, Dordrecht. p. 97-98.

Roth, J., Bendayan, M., Carlemalm, E., Villinger, W., and Garavito, M. 1981. Enhancement of structural preservation and immunocytochemical staining in low temperature embedded pancreatic tissue. J. Histochem. Cytochem. 29: 6633-69.

Roth, J., Bendayan, M., and Orci, L. 1978. Ultrastructural localization of intracellular antigens by the use of Protein A-gold complex. J. Histochem. Cytochem. 26: 1074-81.

Rudnick, P., Kunz, C., Gunatilaka, M.K., Hines, E.R., and Kennedy, C. 2002. Role of GlnK in NifL-Mediated Regulation of NifA Activity in Azotobacter vinelandii. J. Bacteriol. 184(3): 812-20.

Sambrock, J., Fritsch, E. F., and Maniatis, T. 1989. Molecular Cloning: A Laboratory Manual, $2^{\text {nd }}$ ed. Cold Spring Harbor, NY: Cold Spring Harbor Laboratory Press.

Saunders, N.F., Houben, E.N., Koefoed, S., de Weert, S., Reijnders, W.N., Westerhoff, H.V., De Boer, A.P., and Van Spanning, R.J. 1999. Transcription regulation of the nir gene cluster encoding nitrite reductase of Paracoccus denitrificans involves NNR and NirI, a novel type of membrane protein. Mol. Microbiol. 34(1): 24-36.

Sawers, G. 1993. Specific transcriptional requirements for positive regulation of the anaerobically induceable ffl operon by ArcA and FNR proteins. J. Bacteriol. 174: 347478.

Sawers, G., and Suppmann, B. 1992. Anaerobic induction of pyruvate formate-lyase gene expression is mediated by the ArcA and FNR proteins. J. Bacteriol. 174: 3474-78. 
Schmitz, R.A., Klopprogge, K. and Grabbe, R. 2002. Regulation of nitrogen fixation in Klebsiella pneumoniae: NifL, transducing two environmental signals to the nif transcriptional activator NifA. J. Mol. Microbiol. Biotech. 4(3): 235-42.

Schmitz, R.A. 2000. Internal glutamine and glutamate pools in Klebsiella pneumoniae grown under different conditions of nitrogen availability. Curr. Microbiol. 41: 357-62.

Schmitz, R.A. 1997. NifL of Klebsiella pneumoniae carries an N-terminally bound FAD cofactor, which is not directly required for the inhibitiory function of NifL. FEMS Microbiol. Lett. 157: 313-18.

Schmitz, R.A, He, L., and Kustu, S. 1996. Iron is required to relieve inhibitory effects of NifL on transcriptional activation by NifA in Klebsiella pneumoniae. J. Bacteriol. 178: 467987.

Scott, C., Guest, J.R., and Green, J. 2000. Characterization of the Lactococcus lactis transcription factor FlpA and demonstration of an in vitro switch. Mol. Microbiol. 35: 1383-93.

Shaw, D.J., and Guest, J.R. 1982. Nucleotide sequence of the fnr gene and primary structure of the Fnr protein of Escherichia coli. Nucleic Acids Res. 10: 6119-30.

Sidoti, C., Harwood, G., Ackerman, R., Coppard, J. and Merrick, M. 1993, Characterization of mutations in the Klebsiella pneumoniae nitrogen fixation regulatory gene nifL which impair oxygen regulation. Arch. Microbiol. 159: 276-81.

Silhavy, T.J., M. Bermann, and L. W. Enquist. 1984. Experiments with Gene Fusions, p.10712. Cold Spring Harbor, NY: Cold Spring Harbor Laboratory Press.

Skorupsky, K., and Taylor, R.K. 1996. Positive selection vectors for allelic exchange. Gene 169: 47-52.

Skotnicki, M. L., and B. G. Rolfe. 1979. Pathways of energy metabolism required for phenotypic expression of $n i f_{K p}^{\dagger}$ genes in Escherichia coli. Aust. J. Biol. Sci. 32: 637-49.

Söderbäck, E., Reyes-Ramirez, F., Eydmann, T., Austin, S., Hill, S., and Dixon R. 1998. The redox and fixed nitrogen-responsive regulatory protein NifL from Azotobacter vinelandii comprises discrete flavin and nucleotide-binding domains. Mol. Microbiol. 28: 179- 92.

Souza, E.M., Pedrosa, F.O., Drummond, M., Rigo, L.U., and Yates, M.G. 1999. Control of Herbaspirillum seropedicae NifA activity by ammonium ions and oxygen. J. Bacteriol. 181: 681-84.

Spiro, S. 1994. The FNR family of transcriptional regulators. Antonie Van Leeuwenhoek 66: 23-36. 
Spiro S., and Guest, J.R. 1990. FNR and its role in oxygen-regulated gene expression in Escherichia coli. FEMS Microbiol. Rev. 6: 399-428.

Spiro, S., Roberts, R.E., and Guest, J.R. 1989. FNR-dependent repression of the $n d h$ gene of Escherichia coli and metal ion requirement for FNR-regulated gene expression. Mol. Microbiol. 3(5): 601-8.

Spiro, S., and Guest, J.R. 1988. Inactivation of the FNR protein of Escherichia coli by targeted mutagenesis in the N-terminal region. Mol. Microbiol. 2: 701-07.

Steenhoudt, O., and Vanderleyden, J. 2000. Azospirillum, a free-living nitrogen-fixing bacterium closely associated with grasses: genetic, biochemical and ecological aspects. FEMS Microbiol. Rev. 24: 487-506.

Stevanin, T.M., Ioannidis, N., Mills, C.E., Kim, S.O., Hughes, M.N., and Poole, R.K. 2000. Flavohemoglobin Hmp affords inducible protection for Escherichia coli respiration, catalyzed by cytochromes bo' orbd, from nitric oxide. J. Biol. Chem. 275(46): 3586875.

Taylor, B.L. and Zhulin, I.B. 1999. PAS domains: internal sensors of oxygen, redox potential, and light. Microbiol. Mol. Biol. Rev. 63(2): 479-506.

Tran, Q.H., and Unden, G. 1998. Changes in the proton potential and the cellular energetics of Escherichia coli during growth by aerobic and anaerobic respiration or by fermentation. Eur. J. Biochem. 251: 538-43.

Tran, Q.H., Bongaerts, J., Vlad, D., and Unden, G. 1997. Requirement for the protonpumping NADH dehydrogenase I of Escherichia coli in respiration of NADH to fumarate and its bioenergetic implications. Eur. J. Biochem. 244(1): 155-60.

Unden, G., Achebach, S., Holighaus, G., Tran, H.G., Wackwitz, B., Zeuner, Y. 2002. Control of FNR function of Escherichia coli by $\mathrm{O} 2$ and reducing conditions. J. Mol. Microbiol. Biotechnol. 4(3): 263-8.

Unden, G., and Bongaerts, J. 1997. Alternative respiratory pathways of Escherichia coli: energetics and transcriptional regulation in response to electron acceptors. Biochim. Biophys. Acta. 1320(3): 217-34.

Unden, G., and Schirawski, J. 1997. The oxygen-responsive transcriptional regulator FNR of Escherichia coli: the search for signals and reactions. Mol. Microbiol. 25: 205-10.

Unden, G., Becker, S., Bongaerts, J., Holighaus, G., Schirawski, J., and Six, S. 1995. O⿰㇒⿻二丨冂sensing and $\mathrm{O}_{2}$-dependent gene regulation in facultatively anaerobic bacteria. Arch. Microbiol. 164: 81-90. 
Upchurch, R.G., and Mortenson, L.E. 1980. In vivo energetics and control of nitrogen fixation: Changes in the adenylate energy charge and adenosine 5'diphosphate/adenosine 5 '-triphosphate ratio of cells during growth on dinitrogen versus growth on ammonia. J. Bacteriol. 143: 274-84.

van Heeswijk, W.C., Hoving, S., Molenaar, D., Stegeman, B., Kahn, D., and Westerhoff, H.V. 1996. An alternative PII protein in the regulation of glutamine synthetase in Escherichia coli. Mol. Microbiol. 21(1): 133-46.

VanHellemond, J.J., and A.G. Tielens. 1994. Expression and functional properties of fumarate reductase. Biochem. J. 304: 321-31.

Vollack, K.U., Hartig, E., Korner, H., and Zumft, W.G. 1999. Multiple transcription factors of the FNR family in denitrifying Pseudomonas stutzeri: characterization of four fnrlikegenes, regulatory responses and cognate metabolic processes. Mol. Microbiol. 31: 1681-94.

Wackwitz, B., Bongaerts, J., Goodman, S.D., and Unden, G. 1999. Growth phase-dependent regulation of nuоA-N expression in Escherichia coli $\mathrm{K}-12$ by the Fis protein: upstream binding sites and bioenergetic significance. Mol. Gen. Genet. 262(4-5): 876-83.

Weidner, U., Geier, S., Ptock, A., Friedrich, T., Leif, H., and Weiss, H. 1993. The gene locus of the proton-translocating NADH: ubiquinone oxidoreductase in Escherichia coli. Organization of the 14 genes and relationship between the derived proteins and subunits of mitochondrial complex I. J. Mol. Biol. 233(1): 109-22.

Whitby, L.G. 1953. A New Method for Preparing Flavin-adenine Dinucleotide. Biochem. J. 54: $437-42$.

Wood, J.M. 1987. Membrane association of proline dehydrogenase in Escherichia coli is redox dependent. Proc. Natl. Acad. Sci. U S A. 84(2): 373-7.

Woodley, P., and Drummond, M. 1994. Redundancy of the conserved His residue in Azotobacter vinelandii NifL, a histidine protein kinase homologue which regulates transcription of nitrogen fixation genes. Mol. Microbiol. 13: 619-26.

Xiao, H., Shen, S. and Zhu, J. 1998. NifL, an antagonistic regulator of NifA interacting with NifA. Science in China (Series C) 41: 303 - 308.

Xu, Y., Cheah, E., Carr, P.D., van Heeswijk, W.C., Westerhoff, H.V., Vasdevan, S., G. and Ollis, D.L. 1989. GlnK, a III-homologue: Structure reveals ATP binding site and indicates how the T-loops may be involved in molecular recognition. J. Mol. Biol. 282: 149-65. 
Young, J.P.W. 1992. Phylogenetic classification of nitrogen fixing organisms. In: Biological nitrogen fixation. G. Stacey, R.H. Burris and H.J. Evans, eds. Chapman \& Hall, New York . p. 43-86.

Zhulin, I.B., Tayler, B.L., and Dixon, R. 1997. PAS domain S-boxes in Archaea, Bacteria and sensors for oxygen and redox. Trends Biochem. Sci. 22: 331-33. 


\section{Curriculum vitae}

\section{Roman Grabbe, Dipl. Biol.}

Born on December, $31^{\text {th }} 1969$ in Helmarshausen, Germany

\section{Education:}

$1976-1980$

$1980-1986$

1986-1989
Primary education in Oberweser

Secondary education at the Marie-Duran-Schule, Bad

Karlshafen

Secondary education at the Albert-Schweitzer-Gymnasium in Hofgeismar

\section{Scientific Background:}

Oct. 1991-Aug. 1998

Mar. 1997-Aug. 1998

Jan. 1999-June 2002
Study of Biology at the Georg-August University, Göttingen Diploma thesis in Microbiology:"Regulation des Isopropylbenzolabbaus in Rhodococcus erythropolis BD2.“ Scientific assistant at the Institute of Microbiology and Genetics, Georg-August University, Göttingen 